Portland State University

PDXScholar

$1-1-1986$

\title{
Electrochemical methods for speciation of inorganic arsenic
}

Karen Ann D'Arcy

Portland State University

Follow this and additional works at: https://pdxscholar.library.pdx.edu/open_access_etds Let us know how access to this document benefits you.

\section{Recommended Citation}

D'Arcy, Karen Ann, "Electrochemical methods for speciation of inorganic arsenic" (1986). Dissertations and Theses. Paper 524.

https://doi.org/10.15760/etd.524

This Dissertation is brought to you for free and open access. It has been accepted for inclusion in Dissertations and Theses by an authorized administrator of PDXScholar. Please contact us if we can make this document more accessible: pdxscholar@pdx.edu. 
ELECTROCHEMICAL METHODS FOR SPECIATION OF

INORGANIC ARSENIC

by

KAREN ANN D'ARCY

A dissertation submitted in partial fulfillment of the requirements for the degree of

DOCTOR OF PHILOSOPHY

in

ENVIRONMENTAL SCIENCES AND RESOURCES/CHEMISTRY

Portland State University

1986 
TO THE OFFICE OF GRADUATE STUDIES AND RESEARCH:

The members of the Committee approve the dissertation of

Karen Ann D'Arcy presented May 23, 1985.

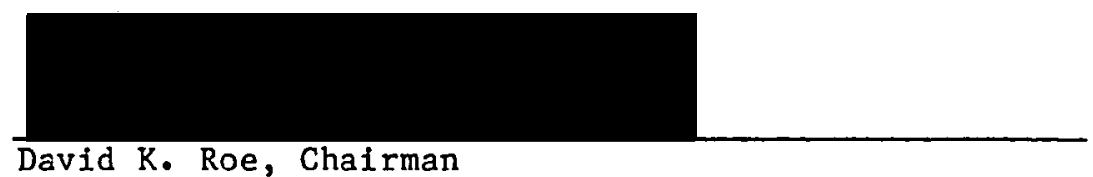

David K. Roe, Chairman
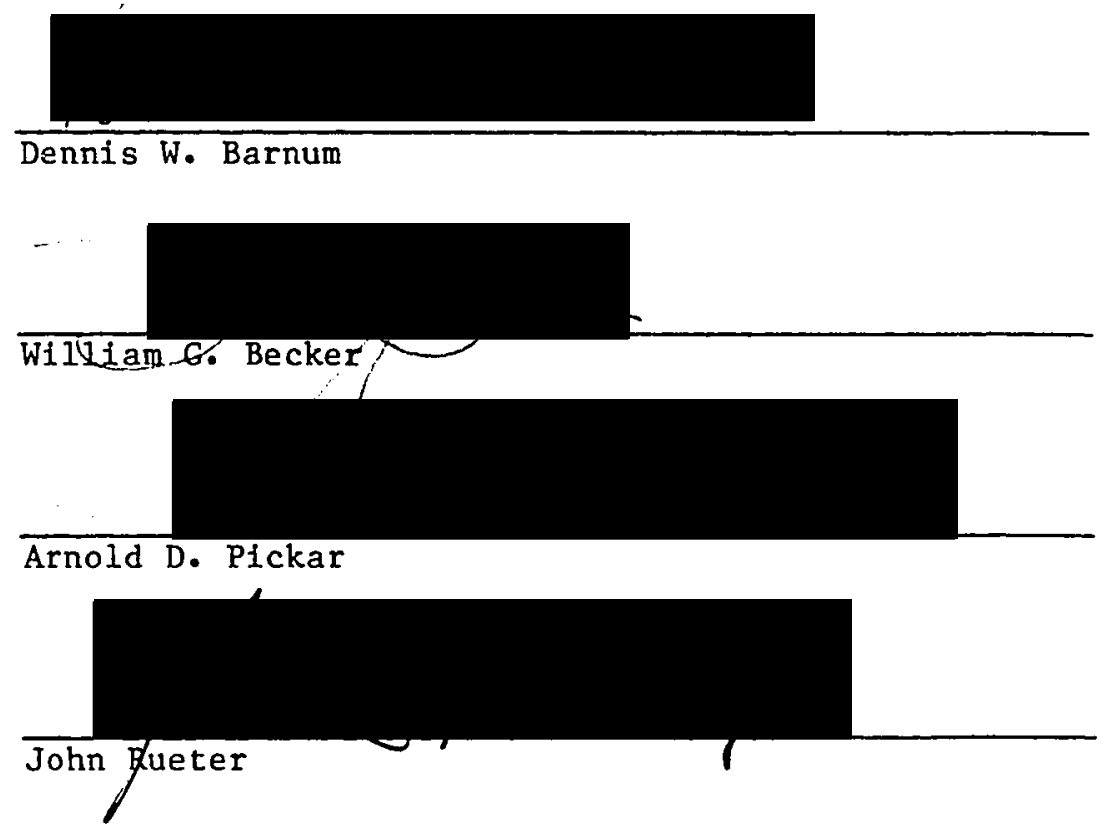

APPROVED:

Pave I K. Smejtek, Environmental science Director

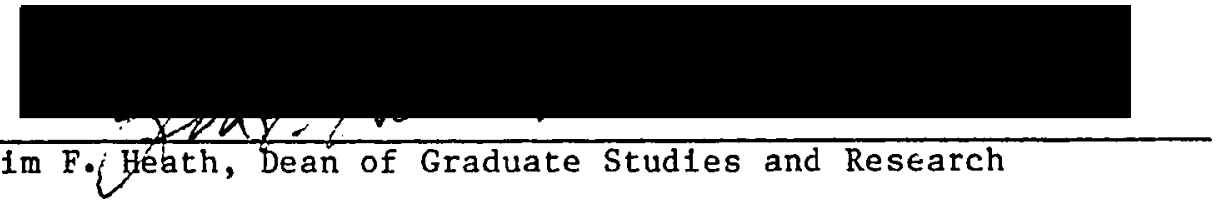


AN ABSTRACT OF THE DISSERTATION OF Karen Ann D'Arcy for the Doctor of Philosophy in Environmental Sciences and Resources/Chemistry presented on May 23, 1985.

Title: Electrochemical Methods for Speciation of Inorganic Arsenic.

APPROVED BY MEMBERS OF THE DISSERTATION COMMITTEE:

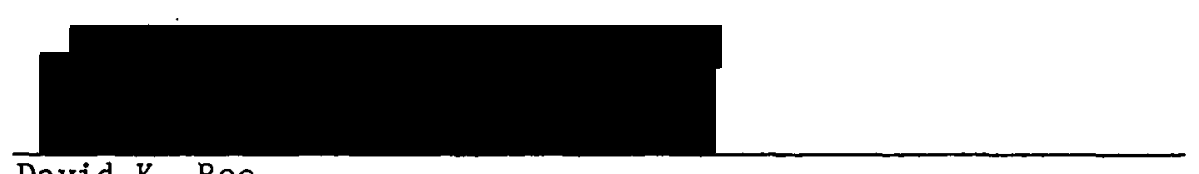
David K. Roe

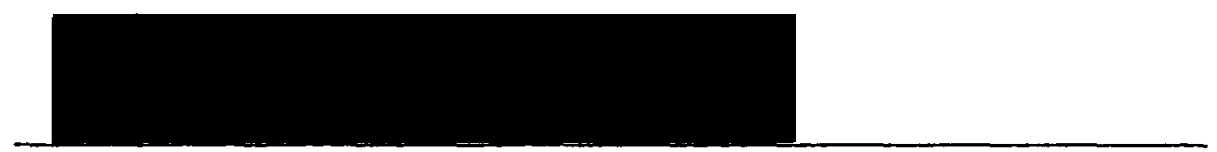
Dennis W. Barnum
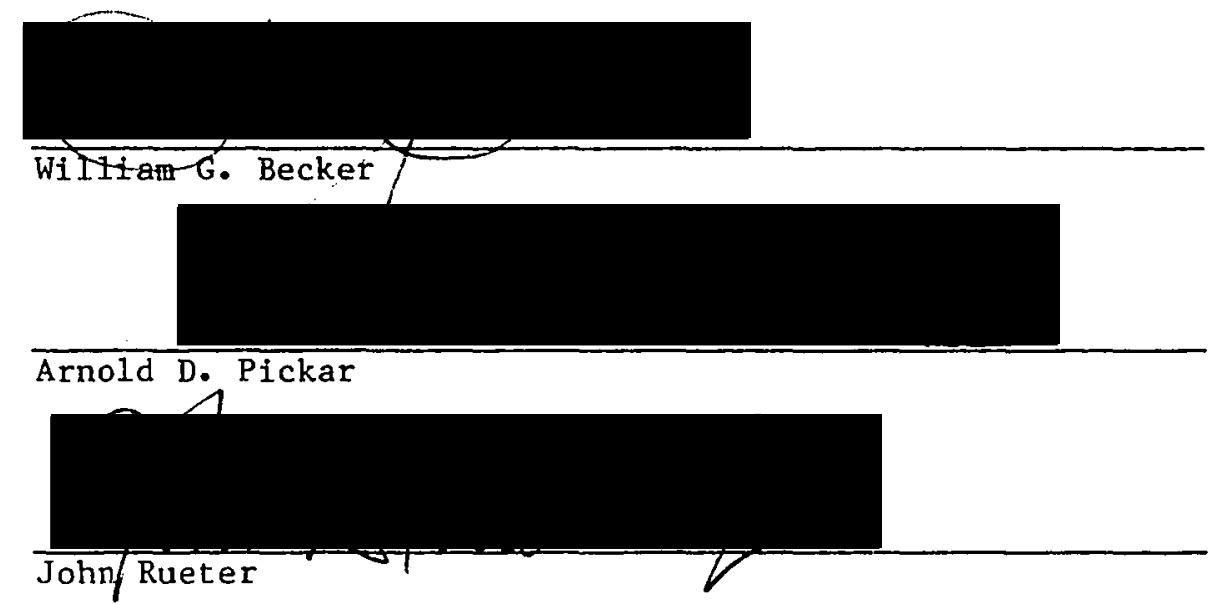

Because of the well-known toxic properties of arsenic, many analytical methods have been developed over the years to monitor arsenic at trace levels. In the environment, this element is found in several oxidation states as well as in a variety of organoarsenic compounds. This situation puts additional demands on the analysis in that it is 
desirable to measure the amount of each species, not just ali of the arsenic. The reason for this is that the different species have greatly different toxicities; of the major inorganic forms, As(III) is much more toxic than As(V). The goal of this research was to develop a convenient method for the analysis of mixtures of As(III) and As(V) at trace levels.

Electroanalytical methods are inherently sensitive to oxidation states of elements and therefore are a natural choice for this problem. In fact, a method was developed some years ago for As(III) that used differential pulse polarography: the detection limit is 0.3 parts per billion ( $(p p b)$. However, As(V) was not detected since in its usual form as an oxyanion it is electrochemically inactive. There are coordination compounds formed with catechol, AsL $(n=1-3)$, that can be reduced at a mercury electrode, but the active species, AsL, is only a small fraction of the major species, AsL, so the detection limit is only 500 ppb. Many details of the electrochemistry of this unusual compound were examined in this work.

In order to improve detection limits, a method involving cathodic stripping was developed. It involves codeposition of copper with arsenic on a mercury electrode to effectively concentrate the analyte. Then the elemental arsenic is converted to arsine, AsH, during a cathodic potential scan. The resulting current peak is proportional to As(III) in the absence of catechol and to the sum of As(III) and As(V) In the presence of catechol. It was observed tiat the current peak was considerably larger than expected and additional experiments revealed that there was evolution of hydrogen during the formation of arsine. 
This is rather unusual in electrochemical reactions and so some of the details of this catalyzed coreaction were examined. The result is a fortunate enhancement of detection limit so that $\mathrm{As}(\mathrm{V})$ at $40 \mathrm{ppb}$ can be measured. 
ACKNOWLEDGEMENTS

I would like to thank the fine faculty and staff of the Chemistry Department of Portland State University. They made my studies challenging, enriching, and enjoyable. I would like to especially thank Dr. David K. Roe. His guidance, patience, and knowledge were the stimulus for this work. I would like to thank Dr. Bruce Brown and his wife Barbara for their encouragement and friendship. Dr. Brown and I endured many long miles and tall tales. The camaraderie I shared with my fellow graduate students was supportive. Mario Aparicio was always ready to answer my questions, give advice or challenge me to run that last mile.

I would like to thank my colleagues at Governors State University for their confidence and encouragement, especially Dr. Phyllis Klingensmith. My parents, Marie and Tom, always provided encouragement and support. I am happy that they are proud of my accomplishment. And finally, Bruce Dumdei cajoled, threatened, and encouraged me to finish. His teasing concern, rivalry, compasion, and affection made the difference. 


\section{LIST OF TABLES}

TABLES

PAGES

I Experimental Conditions for Cathodic Stripping

Voltammetry with Copper Codeposition . . . . . 39

II Reaction of $2.0 \mathrm{mM} \mathrm{Cu(II)}$ and $0.5 \mathrm{mM} \mathrm{As}(\mathrm{V}) \ldots . .81$

III Data and Results of Equilibrium Constant

Calculations Using Nicholson and Shain (52)

Diagnostic Criteria . . . . . . . . 107

IV Data From Kinetic Plots of Decay Experiments

Conducted on Solutions of $1.25 \mathrm{mM}$ (94ppm) As(V). - 110 
1. Biological Cycle for Arsenic .........

2. Differential Pulse Polarogram of $A s(V)$ in $0.5 \mathrm{M}$ Catechol, $\mathrm{pH}=1 . . . . . . . . .$.

3. Linear Potential Scan of $\mathrm{As}(\mathrm{V})$ in $0.5 \mathrm{M}$ Catechol,

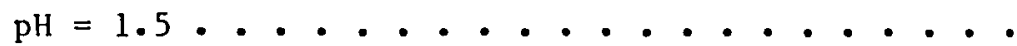

4. Repeated Potential Scans of the Decay of the Electroactive Complex. . . . . . . . .

5. Cyclic Potential Voltammogram of the Electroactive Complex . . . . . . . . . . . . .

6. Linear Potential Scans of the Decay of the Electroactive Complex at Scan Rates of: 200,100 , 50 , and $10 \mathrm{mV} / \mathrm{s} \cdot \ldots \cdot \ldots$

7. Differential Pulse Polarogram of $A s($ III) in $0.5 \mathrm{M}$ Catechol ................

8. Differential Pulse Polarograns of a Series of As(III) Solutions with $0.5 \mathrm{M}$ Catechol. . . .

9. Reduction Current for the Decay of the EAC at Applied Potential of -0.4 v. . . . . . .

10. Differential Pulse Cathodic Stripping of As(III) in $0.5 \mathrm{M}$ catechol ............

11. Differential Pulse Cathodic Stripping of As(V) in 
$0.5 \mathrm{M}$ Catecho1. Depostion Potential $=-0.5 \mathrm{~V} \cdot$.

12. Differential Pulse Cathodic Stripping of $\Lambda s(V)$ in $0.5 \mathrm{M}$ Catechol. Deposition Potential $=-0.3 \mathrm{~V}$.

13. Current Voltage Curves for Integration Studies . • 46

14. Charge Voltage Curves for Integration Studies • • 48

15. Effect of Catechol on the Reduction of Cu(II). . 50

16. Differential Pulse Cathodic Stripping of $370 \mathrm{ppb}$ As(V) with $\mathrm{Cu}(\mathrm{I})$ codeposition in $0.5 \mathrm{M}$ Catechol. .

17. Cathodic Stripping Performed at a CopperAmalgam Electrode. . . . . . . . . .

18. Current Decay of the EAC. Reduction Peak Current as a Function of Time, $\mathrm{pH}=0 . . . . . . . \cdot$

19. Kinetic Plot of the EAC Decay Current. . . . . 66

20. Current Decay of the EAC. Reduction Peak Curent as a Function of Time. $\mathrm{pH}=2 . \ldots . . . .$.

21. Initia1 Current, $i$, as a Function of Catechol Concentration in $1.0 \mathrm{~N} \mathrm{HCl}$ Solution with $94 \mathrm{ppm}$ As(V)

22. Initial Current, $i$, as a Function of Catechol Concentration in $0.1 \mathrm{~N} \mathrm{HCl}$ Solution with 94 ppm As(V) 70

23. Calibration Curve For Analysis of $\Lambda s$ (III) in $0.5 \mathrm{M}$ Catechol Solution by Differential Pulse Polarography. $0 \mathrm{ppb}$ to $200 \mathrm{ppb} \cdot$. . . . . . . . •

24. Calibration Curve for Analysis of As(III) in $0.5 \mathrm{M}$ Catechol Solution by Differential Pulse Polarography.

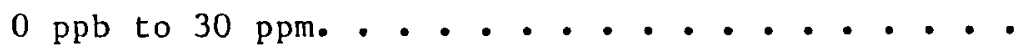


25. Ca1ibration Curve for Analysis of As(V) in $0.5 \mathrm{M}$ Catechol Solution, Determined by Constant Potential Voltammetry. Eapplied $=-0.4 \mathrm{~V} \cdot . . . . . .$.

26. Dependence of Cathodic Stripping Current on Cu(II) Concentration . . . . . . . . . . . . 76

27. Dependence of Normalized Stripping Enhancement, (DIF)/(As), on Normalized Copper Concentration, $[\mathrm{Cu}] /[\mathrm{As}] \cdot \cdot \cdot \cdot \cdot \cdot \cdot \cdot \cdot \cdot \cdot \cdot \cdot \cdot \cdot \cdot \cdot \cdot \cdot \cdot 78$

28. Calibration Curve for $\Lambda s($ III), Step I Results. • . 83

29. Calibration Curve for $A s$ (III) as Determined by the Two Step Method for Inorganic Arsenic Speciation. Step II results. . . . . . . . . . . . .

30. Current Decay of the EAC Monitored by Cathodic Stripping Voltammetry with Copper Codeposition . • . 86

31. Calibration Curve for $A s(V)$ Determined by the Two Step Method for Inorganic Arsenic Speciation. Step II Results. . . . . . . . . . . . . 88

32. Calibration Curve for Inorganic Arsenic Mixture. $[\Lambda s(V)]=667$ ppb. Step I Results. . . . . . . 89

33. Calibration Curve for Inorganic Arsenic Mixture, Step II Results. $[\Lambda \mathrm{s}(\mathrm{V})]=346 \mathrm{ppb},[\Lambda \mathrm{s}(\mathrm{III})]=$ Variable

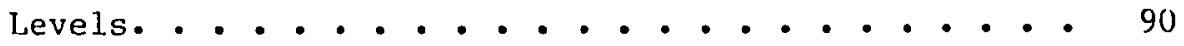

34. Calibration Curve for Inorganic Arsenic Mixture, Step I Results. $[A s(I I I)]=78 \mathrm{ppb} \cdot$...$\cdot$. . 92

35. Calibration Curve for Inorganic Arsenic Mixture, 
Step II Results. [As(III)] $=37.4 \mathrm{ppb},[\mathrm{As}(\mathrm{V})]=$ Vartiable Levels. . . . . . . . . . .

36. Ratio of Kinetic Current to Diffusion Current as a Function of the Inverse of the Square Root of Scan Rate, Experiments Conducted in $0.1 \mathrm{~N} \mathrm{HCl} .103$

37. Ratio of Kinetic Current to Diffusion Current as a Function of the Inverse of the Square Root of Scan Rate, Experiments Conducted in $1.0 \mathrm{~N} \mathrm{HCl}$. . . . 104 


\section{INTRODUCTION}

Arsenic is an ubiquitous element in the environment. Typical environmental concentrations range from the low parts per billion, $(\mathrm{ppb})$, to several parts per million, (ppm), in water, soil, and biolngical samples. It's toxicity is well known (1). Arsenic is important in a number of industrial applications. Arsenic compounds are used extensively in agriculture as herbicides (1). Until recently arsenic compounds were used as wood preservatives. It is also a feed additive used in the poultry industry. Through manufacturing and agricultural use, arsenic and its compounds are introduced into the environment. To protect human health the Environmental Protection Agency (2) has set the drinking water standard at $50 \mathrm{ug} / 1$, or ppb, of arsenic and Occupational Safety and Health Agency ( 3 ) has established an atmospheric standard of $10 \mathrm{mg} / \mathrm{m}^{3}$ for inorganic arsenic in the workplace.

The International Agency for Research on Cancer, (IARC), (4) has stated that "... there is sufficient evidence that inorganic arsenic compounds are skin and lung carcinogens in humans." In contrast there is inadequate evidence to prove that arsenic compounds are carcinogenic in experimental animals (4). Arsenic appears to be a uniquely human carcinogen although experimental animal models are lacking. This unique characteristic of arsenic has fostered significant controversy $(4,5,6,7)$. Without an experimental animal model to study, fundamental research which could evaluate the effects of chronic exposire and dosage of various arsenic compounds found in the environment cannot be conducted. The lack of this information prevents the regulatory 
agencies from being confident of their established water and air quality standards.

Arsenic is found in the environment in a variety of chemical forms $(8,9)$. The most common species are arsenate, As(V), arsenite, As(III), monomethylarsonic acid, (MMA), and dimethylarsenic acid, (DMA). The concentration of the various species depends on the environmental characteristics such as aerobic or anaerobic conditions, pll, moisture, and biological activity. Inorganic forms of arsenic, As(III) and As(V), are reduced and often methylated to form dimethylarsine by methanogenic bacteria $(1,9)$. The intermediates in this process are monomethylarsonic acid and dimethylarsenic acid. The result of the anaerobic reduction and methylation is production of volatile, toxic arsines. The volatile products evaporate from soil or water resulting in removal of the arsenic from the organisms' microenvironment. This represents a detoxification of the microenvironment of the anaerobes. The volatile arsines are readily oxidized and may deposit onto the soil or water system. In Figure 1, a diagram from Wood (9), the biological cycle for arsenic is shown. The various arsenic species found in the environment possess differing toxicities (1). The inorganic forms, As(III) and As(V), are much more toxic than the organic species (MMA, DMA). Arsenite is the most toxic species of significant concentration in natural water systems.

A number of studies have been conducted which survey water systems, soil samples and biological specimens for the concentration of the various arsenic species $(8,10)$. It has been found that arsenate, $A s(V)$, is the predominant species in oxidizing, aerobic environments 


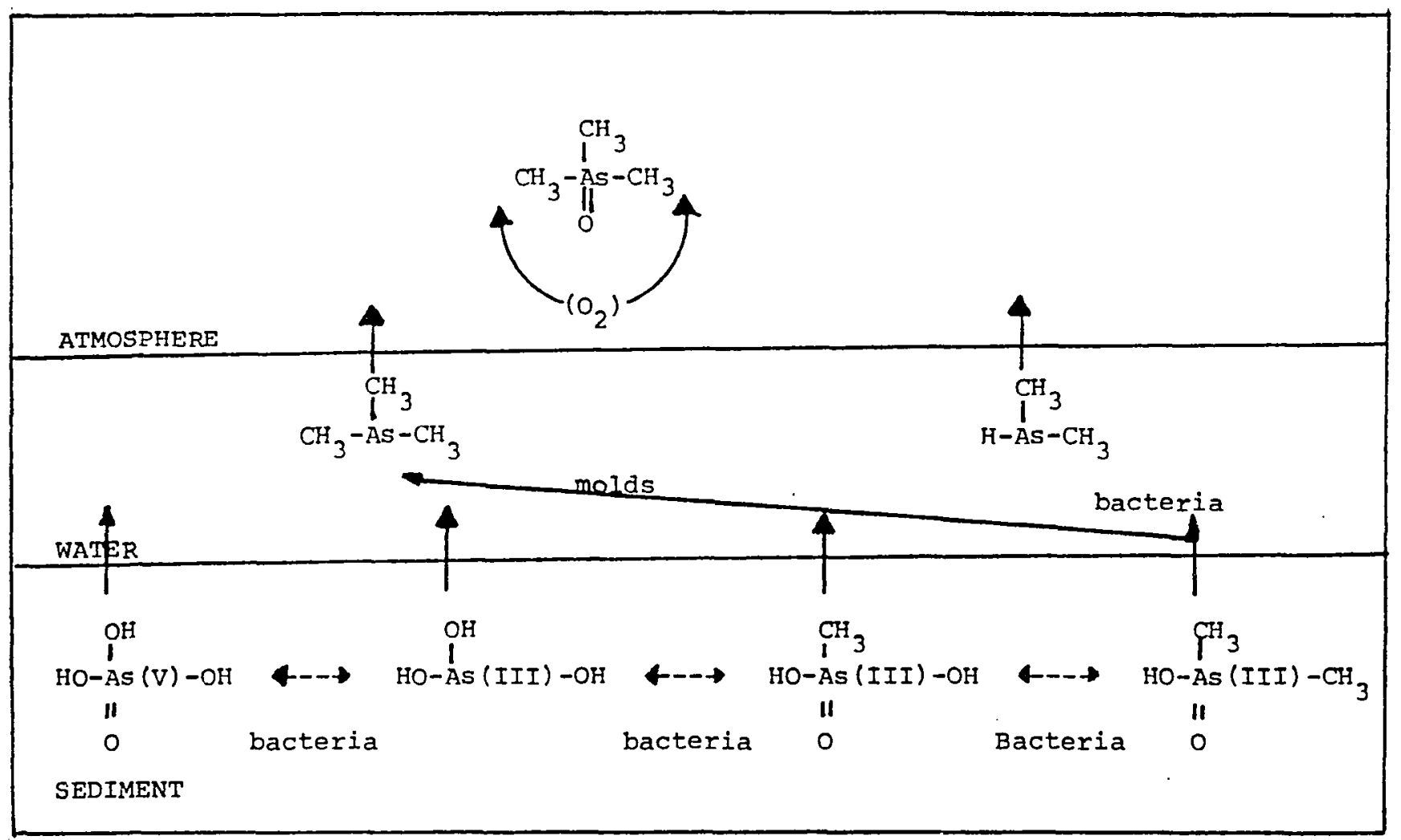

Figure 1. Biological cycle for arsenic. 
while arsenite, As(III), is the predominant species in reducing, anaerobic conditions. Of the organic arsenic species, DMA is the predominant form, often present in concentrations of $50 \%$ or more of the total arsenic in biological samples such as human urine. In marine studies DMA is the predominant organic species, typically present in concentrations of $10 \%$ to $15 \%$ of the total arsenic. Monomethylarsonic acid is the minor component, usually present as less than $1 \%$ of the total arsenic in a sample $(8,10)$.

The concentration of arsenite and arsenate reflect the oxidationreduction potential of the water sample in which they are measured. The evaluation of the oxidation-reduction potential ( $\mathrm{pE}$ or $\mathrm{Eh}$ ) is a measure of the electron activity of a solution and is defined analogous to $\mathrm{pH}$. The concept of $\mathrm{pE}$ is a valuable tool for interpretation of the chemistry of hydro-chemical systems. The measurement of the oxidation-reduction potential is performed with potential sensing electrodes, redox indicator species, or redox couples. The As(III)-As(V) couple is useful to evaluate the redox potential in a redox window (range of $\mathrm{pE}-\mathrm{pH}$ values) with important application to groundwater studies (11). In particular the As(III)-As(V) couple is especially useful because the kinetics of interconversion between $A s($ III) and $A s(V)$ are sufficiently slow to permit collection, transportation and analysis before significant change in species distribution can occur. The $\Lambda s($ III) $\longleftrightarrow->$ $A s(V)$ interconversion can equilibrate on a geologic time scale so that the arsenic redox couple does reflect the redox potential of a ground water sample. Unfortunately convenient techniques for the direct measurement of both $A s(I I I)$ and $A s(V)$ have not been developed. Arsenic 
is also the inorganic substituent which most often exceeds the maximum permissible drinking water limits in ground water samples. Because the typical arsenic concentration is sufficiently high to permit measurement in most samples and the useful position of its redox window, arsenic is we11 suited for use as a redox indicator in groundwater sample (11). Sensitive and selective analytical techniques are needed to measure the concentration of the various arsenic species in environmental samples. Environmental monitoring and the measurement of pesticide residues, toxilogical studies, the study of the mechanism of biological conversion and metabolism, and the application of the arsenic redox couple to determine the redox potential of a water sample all require powerful analytical techniques which provide speciation information. Many methods have been developed for analysis of arsenic concentration $(8-44)$. Many techniques measure the total arsenic concentration which satisfies the national drinking water standard which is set at $50 \mathrm{ug} / \mathrm{L}$ or ppb total arsenic (12 - 14). 0ther techniques separate and quantify the various inorganic and organic arsenic species found in water, sediment, and biological samples. The measurement of the various arsenic species provides more information with which evaluation of the arsenic toxicity or hazard of any given sample can be made.

Analytical methods have been developed for determination of arsenic concentration in water samples and similar matrices. The molybdenum blue method and the silver diethyldithiocarbamate method are spectrophotometric techniques which measure the absorbance of arsenic complexes $(12,13,14)$. These techniques have limited sensitivity, often 
require experienced analysts, and are plagued by a number of interferences.

Atomic absorption spectroscopy, (AA), has been successfu11y applied to arsenic analysis. The typical flame atomization source for AA analysis is relatively insensitive to arsenic concentration (15). Arsine generators and flameless atomization techniques such as graphite furnace atomizers extend the detection limit of arsenic to the sub parts per billion range (13). The flameless $A$ A techniques are sensitive but their results indicate the total arsenic concentration and do not distinguish between various arsenic species. Other methods have been developed which couple separation steps with flameless AA analysis. Several methods have been developed which use extraction techniques to separate the arsenic species followed by quantification using flameless AA $(16,17)$

Recent techniques have coupled the separation powers of liquid chromatography and the quantification of $A A$ spectroscopy to speciate inorganic and organic arsenic. The method of Brinkman et a1. (18) utilizes a variety of columns to separate the four predomonant arsenic species resulting in identification and quantification of the arsenic species in solutions containing $10-20$ ppb arsenic (18). This technique requires extensive instrumentation and results in lengthy analysis time. Iverson et a1. (19) have developed another column chromatography-flameless $M$ spectroscopic technique for speciation of inorganic arsenic, MMA and DMA. Reported detection limits are 2 ppb of arsenic. This technique is not automated and requires collection of column eluant followed by flameless AA analysis of the fractions. This 
technique does not distinguish between the two inorganic arsenic species. An automated technique which couples high pressure liquid chromatography to flameless AA was developed by Woolson and Aharonson (20). All four predominant arsenic species are separated and quantified by this method with sensitivities of $0.25 \mathrm{ppm}$ arsenic for each species. This technique is more convenient but lacks the required sensitivity for envi ronmental sample analysis.

A number of methods combine ion chromatography and AA spectroscopy for separation and quantification of the various arsenic species. Elaborate elution sequences or individual separations are required for some species $(21,22)$. Atomic absorption spectroscopy has also been combined with hydride generation for analysis of water samples and biological samples (23).

Inductively coupled plasma, (ICP), atomic emission spectroscopy is a common method for arsenic detection. A number of analytical schemes can determine total arsenic content simultaneously with other elements in water, biological samples, and sediment $(24,25)$. Liquid chromatography has also been combined with ICP to speciate and quantify the arsenic compounds in biological samples (26). These techniques often fail to combine speciation with enough sensitivity and require extensive instrumentation.

Hydride generation is a very popular technique for removal of arsenic compounds from sample matrices, or as a technique to chemically modify the arsenic compound for quantification. Many of the early arsenic speciation studies employed hydride generation followed by fractional evaporation and quantification of the various arsines 
produced. Braman and Foreback ( 8 ) were the first researchers to report the concentration of the various arsenic species in studies of water samples and biological samples. The technique of Foreback and Braman (8) depends on $\mathrm{pH}$ selective reduction of the various arsenic forms with sodium borohydride followed by separation of the volatile arsines produced by selective volatilization from a cold trap. The arsines are detected by atomic emission from an electrical discharge.

Various chromatographic methods have been used to separate the arsenic species followed by detection using atomic absorption spectroscopy (as discussed above), ICP emmission spectroscopy, and mass spectroscopy. Odanaka et al. (27) have developed a method for GC-MS determination of inorganic arsenic and methyl arsine following hydride generation. Reported detection limits were $0.2-0.4 \mathrm{ppb}$.

Electrochemical methods are well known for their sensitivity and selectivity. As(III) is electroactive and several siccessful analyses have been developed using differential pulse polarography (28). As(V) is electroinactive in the absence of certain complexing agents and its presence is not detected in typical polarographic experiments. Many organic arsenicals are electroactive, including DMA and MMA, the polarography of these compounds has been reported $(29,30)$. The electrochemical detection limits for the organic arsenic compounds are poor. Electrochemical methods are best suited for analysis of As(III) providing a selective and sensitive technique $(31,32,33)$.

Differential pulse polarographic, DPP, analysis of As(III) concentration has been successfully developed by Myer and 0steryoung (28). The reported detection limit is $0.3 \mathrm{ppb}$. This method has been 
applied to the analysis of water, sewage and sludge (33). The technique of DPP has been used to speciate inorganic arsenic (34). The As(III) concentration was measured directly by DPP, then the water sample was treated with sodium bisulfite to reduce the As(V) to As(III) and a DPP analysis was performed again to measure total inorganic arsenic. The As $(V)$ concentration is calculated by difference, subtracting the As(III) measurement from the total inorganic arsenic measurement. Henry and Thorpe (35) further exploited the power of differential pulse polarography to speciate a water sample for organic and inorganic arsenic. Ion exchange chromatography was used to separate the species followed by digestion of the organic arsenic species and conversion to As(III) for quantification by DPP. Inorganic arsenic speciation can be - determined by the method of Henry, Thorpe, and Kirch (34) as described above. This speciation technique results in detection limits of $18 \mathrm{ppb}$ and $8 \mathrm{ppb}$ for MMA and DMA respectively. This method is fairly complex and time consuming.

In differential pulse polarography the analytical signal is the current produced by reduction of As(III) to the elemental state. The oxidation of As(III) to $A s(V)$ has also been used to produce a useful analytical signal. Cox and Kulesza (36) have published a technique to determine the arsenite concentration through electrooxidation at a glassy carbon electrode modified with a thin film of mixed-valence ruthenium (III,II) cyanide. The linear analytical range is $0.4-150$ ppm•

Aside from the speciation techniques developed by Henry et al. $(34,35)$, electrochemical analyses have focused on analysis of the 
As(III) ion. Several voltammetric stripping techniques have been developed for As(III) analysis, to take advantage of the sensitivity and selectivity of stripping methods. Anodic, potentiometric, and cathodic stripping techniques have been reported in the literature, $(37-44)$.

Anodic stripping techniques, (ASV), have been developed which are selective for $A s(I I I)$. The $A s(I I I)$ is reduced to the elemental state at the working electrode which is composed of either gold or platinum (37). The deposit is then oxidized to form a soluble arsenic species. The analytical signal is produced by the oxidation of the deposited arsenic as the deposit is stripped from the electrode. Forsberg, et al. (37) reported the use of both platinum and gold working electrodes in anodic stripping analysis of As (III) (34). Gold electrodes were found to have a higher hydrogen overvoltage then platinum electrodes providing a decided advantage. The influence of pH, deposition potential, supporting electrolyte, and the nature of the working electrode on the sensitivity and precision of $A s$ (III) analysis were studied (37). The detection limit for As(III) determinations by differential pulse anodic stripping (DPASV) and anodic stripping voltammetry (ASV) was $0.02 \mathrm{ppb}$ for both methods. Total inorganic arsenic can be determined by reduction of the $A s(V)$ species to $\Lambda s$ (III) followed by anodic stripping voltammetry. A similar technique was reported by Wang, et a1. (39). Thin gold-film electrodes have also been used as working electrodes for As(III) anodic stripping analysis. Davis, et al: (40) have developed a method which includes sample digestion, reduction and distillation of the arsenic from the sample as $\mathrm{AsCl}$ vapors and anodic stripping analysis of the resulting solution. The anodic stripping 
analysis is a high speed technique characterized by good precision and accuracy. This technique and a variation reported by Lee and Meranger (41) determine the total arsenic content in samples.

The concentration of As(III) has also been determined by cathodic stripping techniques. In these methods the As(III) is reduced to the elemental state and codeposited with another element onto a mercury working electrode. The stripping step applies a cathodic scan which further reduces the arsenic deposit to Asll . The cathodic stripping signal occurs when the arsenic deposit is reduced to arsine. This technique eliminates a number of interferences encountered in ASV because many of the trace metal ions present in a sample cannot be cathodically stripped.

Holak (42) developed a procedure to determine the As(III) concentration using cathodic stripping voltammetry. From analytical solutions containing $0.36 \mathrm{~N}$ sulfuric acid and $50 \mathrm{ug} / 1$ selenium(IV) the arsenite ion can be deposited onto a hanging mercury drop electrode (HMDE) and cathodically stripped. As(III) concentrations of 2 ppb can be detected after 1.5 minute deposition periods.

Another cathodic stripping technique was developed by Henze, Joshi and Neeb (43). A HIME was again used as the working electrode. The analytical solution contained $1.0 \mathrm{~N} \mathrm{HCl}, 0.002 \mathrm{M}, 127 \mathrm{ppm} \mathrm{CuCl}$, and As(III). A potential of $-0.55 \mathrm{~V}$ is applied for the deposition period, followed by differential pulse cathodic stripping to produce a peak at $0.77 \mathrm{~V}$ versus $\mathrm{Ag} / \mathrm{AgCl}$ reference electrode. The dependence of peak height on the arsenic concentration is linear in the rather narrow range of 0.2 $\mathrm{ppb}$ to $20 \mathrm{ppb} \mathrm{As}(\mathrm{III})$. 
Sadana (44) developed a cathodic stripping technique similar to the method developed by Henze et al. (43). The arsenic(III) concentration was determined by cathodic stripping at a hanging mercury drop electrode. The sample was acidified to $0.75 \mathrm{M} \mathrm{HCl}$ and contained a $\mathrm{Cu}(\mathrm{II})$ concentration of $5 \mathrm{ppm}$. The deposition potential of $-0.6 \mathrm{~V}$ was applied for 120 seconds followed by a 30 second equilibration. The analysis was accomplished by a differential pulse cathodic scan at the rate of $8 \mathrm{mV} / \mathrm{s}$ to produce a stripping peak at $-0.72 \mathrm{~V}$ vs. $\mathrm{Ag} / \mathrm{AgCl}$ reference electrode which was proportional to the arsenic concentration. The reported detection limit is $1 \mathrm{ppb}$ As(III).

The many analytical methods developed for arsenic determination and speciation supply quantitative results for a variety of applications. Many techniques simply measure the total arsenic present, providing data to satisfy the drinking water standards. Other techniques separate the various species and determine their respective concentrations to provide data useful in environmental monitoring, modelling, toxicity studies, and biological metabolism studies. Most of these techniques do not measure arsenate, $A s(V)$, directly, but determine its concentration by the method of difference. The As(III) concentration is measured, and the $A s(V)$ is converted to $A s($ III) and a new concentration is measured which corresponds to the total inorganic arsenic. The arsenate concentration is then calculated to be the difference between the total inorganic arsenic and the arsenite concentration. Indirect methods are often subject to contamination and analyte loss. A method of directly determining the As(V) concentration would be useful in speciation studies and for application of the arsenic 
redox couple to determination of ground water oxidation-reduction potential.

Electrochemical techniques are very selective and sensitive for As(III). The detection limits for electrochemical analysis of the organic arsenic species are too insensitive for application to environmental sample analysis. Arsenate is typically electrochemically inert. In the presence of a number of polyols such as pyrogallol (1,2,3-trihydroxybenzene) and catechol (1,2-dihydroxybenzene), arsenate forms an electroactive complex, (EAC), which can be analyzed by polarography (45). The polarography of the As(V)-catechol complex was demonstrated by Roe and Haak $(46,47)$. A number of researchers have studied the complexes of $A s(V)$ and catechol $(48,49,50)$. They have observed that $A s(V)$ forms a series of complexes with catechol that correspond to the molar ratios of $1: 1,1: 2$, and $1: 3$, arsenic to catechol respectively. Various kinetic and equilibrium features of these complexes have been studied. The stability constants reported for these complexes in the literature are not in agreement (47).

An electrochemical method for determination of the As(V) concentration in enviromental samples is reported in this study. In the presence of excess catechol the typically electrolnactive As(V) is converted to an electroactive form which can be observed by electrochemical techniques. The As(V)-catechol system is unusually complex and its successful use in electrochemical analysis requires the study of its chemical features. The technique of linear potential scan voltammetry was used to analyze this complex system and provide details about the formation, reaction and reduction of the EAC. The individual 
details of the $\Lambda s(V)$-catechol system were used to deduce the identity of the EAC. Knowledge of the EAC's identity and of the chemical details of its formation and reaction was used to choose the proper experimental conditions for successful electrochemical analysis. The methods of DPP and cathodic stipping voltammetry were then used for determination of As(V) concentrations. The method of cathodic stripping voltammetry was found to be the most sensitive method for As(V) determination. 


\section{MATERIALS AND APPARATUS}

\section{CHEMICALS}

$\underline{\text { Acids }}$

Dilute hydrochloric and perchloric actds were prepared from high purity Ultrex brand concentrated acids from J. T. Baker Chemfcal Company. Dilute hydrochloric acid solutions were also prepared by isothermal distillation of reagent grade hydrochloric acid from J. T. Baker Chemical Company.

$\underline{\text { As (III) }}$

Arsenite, As(III) solutions were prepared by dissolving Mallinckrodt Primary Standard arsenic trioxide, As 0 , in a minimum of 3 23

$\mathrm{N} \mathrm{NaOH}$, followed by neutralization with $\mathrm{HCl}$ and dilution to volume with triply distilled water or $1.0 \mathrm{~N}$ acid.

$\underline{\operatorname{As}(\mathrm{V})}$

Arsenate, $A s(V)$ solutions were prepared from Baker Analyzed reagent grade arsenic pentoxide in a similar manner to the preparation of As(III) solutions.

Water

The water used in this experiment was prepared from the building supply of delonized or reverse osmosis water by distillation in a quartz 
stili. The water was stored in 1 liter polyethylene bottles.

Catecho1

Catechol of $99 \%$ purity was obtained from Aldrich Chemical

Company, Inc. The catechol was vacuum distilled prior to use.

Nitrogen

Nitrogen was obtained in gas cylinders from Airco.

Copper (II) chloride

Copper (II) chloride, CuCl, was analytical reagent grade and 2

obtained from Mallinckrodt. It was used without further purification.

Copper (I) Chloride

Copper (I) chloride, $\mathrm{CuCl}$, was obtained from J. T. Baker Chemical Company and used without further purification. Solid CuCl was dissolved in a minimum of concentrated $\mathrm{HCl}$ and diluted to volume with water.

Mercury

The mercury metal used for electrodes was triply distilled.

Mercury (II) Chloride

Mercury (II) chloride, $\mathrm{HgCl}_{2}$, was obtained from Mallinckrodt and was used without further purification. 
Sodium Chloride

Reagent grade sodium chloride, $\mathrm{NaCl}$, was obtained from Matheson Coleman and Bell. It was used without further purification.

\section{$\underline{\text { Selenium }}$}

Selenium metal shot was obtained from Matheson, Coleman and Bell. Solutions of Se(IV) were prepared by heating selenium shot with nitric acid to fumes, and dissolving the residue and diluting to volume.

$\underline{\text { Palladium }}$

Solutions of palladium (II) were prepared by dissolving palladium wire in boiling acid and diluting the residue to volume.

Nickel (II) Sulfate

Nickel (II) sulfate was obtained from J. T. Baker Chemical Company and used without further purification.

Iron (III) Sulfate

Iron (III) sulfate was obtained from J. T. Baker Chemical Company and used without further purification.

Sodium Hydroxide

Sodium hydroxide, $\mathrm{NaOH}$, was used to prepare dilute solutions. Reagent grade $\mathrm{NaOH}$ was obtained from J. T. Baker Chemical Company. 
APPARATUS

Polarographic Equipment

Polarographic experiments were conducted with a Princeton Applied Research Polarographic Analyzer, PAR model 174A. A Princeton Applied Research Static Mercury Drop Electrode, PAR model 303, was used as the electrode assembly.

\section{Cathodic Stripping Voltammetry}

The PAR model 303 static mercury drop electrode was operated as a hanging drop electrode for cathodic stripping experiments. The PAR model 303 electrode was powered by the PAR polarographic analyzer, mode1 174A. The model 303 electrode was modified so that the electrochemical experiments conducted at the electrode could be controlled by a separate potentiometer while the PAR model $174 \mathrm{~A}$ polarographic analyzer continued to power the electronic circuitry of the static mercury drop electrode. This arrangement permitted a Heka potentiometer to control operation of the potential wave form applied to the electrode and collect the current and charge data produced in the electrochemical experiment. Charge collection, current integration experiments were conducted with the Heka potentiometer.

\section{Anodic Stripping}

A dual potentiometer and rotating electrode assembly made in this laboratory were used in anodic stripping experiments performed with a rotating gold electrode. 
EXPERIMENTAL METHODS

\begin{abstract}
The electrochemical methods used in this study are well characterized both in theory and practice (51) and many analytical applications have been developed over the past 50 years. Special circumstances arose in the attempts to use these methods with trace levels of $A s(I I I)$ and $A s(V)$ and in this chapter many details of the experimental procedure are described in three sections; Electroactive Complex Studies, Differential Pulse Polarographic Studies of As(III) and As(V) in the Presence of Catechol, and Stripping Voltammetry.
\end{abstract}

\title{
ELECTROACTIVE COMPLEX STUDIES
}

Arsenate, As(V), forms a series of three complexes with catechol, in the proportions of $1: 1,1: 2$, and $1: 3$ arsenic to catechol ratios. At least one of these complexes is electroactive and can be reduced at a mercury electrode to produce a useful analytical signal. One of the goals of this research is to identify the electroactive complex (EAC). Haak (47) reported that the electroactive complex is the $1: 1$ species and he studied the influence of catechol and $\mathrm{pH}$ on the formation of the EAC. Under reaction conditions of low $\mathrm{pH}$ and high catechol concentration the electroactive complex was observed to produce a large polarographic signal at the time of mixing and then as the reaction mixture approaches equilibrium the polarographic signal decayed to a constant value. This indicates that the EAC is not the predominant species at equilibrium. Additional experiments conducted in this study examined the kinetic and 
equilibrium behavoir of the electroactive complex.

Kinetic Studies of the Electroactive Complex

A series of kinetic experiments were conducted to determine the kinetic behavior of the electroactive complex and to clarify its identity. The methods of linear scan voltammetry (LSV) and differential pulse polarography (DPP) were used to monitor the reacting species, the EAC. The kinetic studies were performed in the following manner. The supporting electrolyte was prepared at the desired $\mathrm{pH}$, catechol concentration, and ionic strength. The arsenate ion was added as an aliquot of standard stock solution to produce an analytical

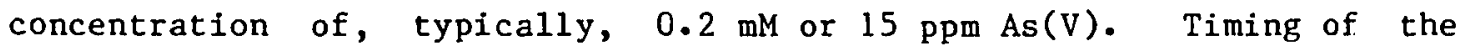
kinetic experiments commenced with the addition of the arsenate aliquot. The reaction mixture was deaerated and stirred for 30 seconds by nitrogen gas bubbling after the As(V) was added. After mixing and dearation the reaction mixture was periodically analyzed by a potential scan - The recording of the scan is presented as a graph of current vs. applied voltage, these graphs will be referred to as current-voltage, (CV), curves. The voltage range scanned initiated at $0.0 \mathrm{~V}$ (versus the $\mathrm{Ag} / \mathrm{AgCl}$ reference electrode) and completed at a potential more negative than the desired reduction peak. The final potential of the scan varied from $-0.5 \mathrm{~V}$ to -1.1 volts. In this voltage range the reduction of the As(V)-catechol electroactive complex starts at approximately $-0.20 \mathrm{~V}$. The height of the reduction wave or peak is directly proportional to the concentration of the electroactive complex at the moment of measurement. The reduction current was measured and used in kinetic calculations to 
evaluate reaction order and rate constants. The reaction rates varied with conditions but were slow enough for manual measurements and the solutions typically reach equilibrium after 0.25 to 2.0 hours.

The current voltage curves recorded for the complete reduction of the As(V)- catechol electroactive complex have three reduction waves or peaks. The following equations very simply illustrate the overall reduction equations, they are not intended to imply which chemical species actually undergoes charge transfer.

$$
\begin{aligned}
& \mathrm{As}(\mathrm{V})+2 \mathrm{e}^{2} \mathrm{As}(\mathrm{III}) \\
& \mathrm{As}(\mathrm{III})+3 \mathrm{e}^{2} \mathrm{As}(\mathrm{O})+3 \mathrm{e}^{-}+3 \mathrm{H}^{+} \mathrm{AsH}
\end{aligned}
$$

The current peaks which correspond to steps 1 or 2 are both suitable for data analysis. The peak for step 3 often appears as a shoulder on the hydrogen evolution wave and is often obscurred by a characteristic polarographic maximum and is unsuitable for data analysis. A typical differential pulse polarogram and a cyclic voltammogram are shown in Figures 2 and 3.

In kinetic studies at low $\mathrm{pH}^{\prime} \mathrm{s}$, the reduction current for the EAC was ai a maximum at the time of reaction initiation. The current then decays to a minimum when equilibrium is reached. During the course of an experiment the concentration of the EAC was monitored by periodic voltage scans at preset time intervals. Figure 4 shows a set of periodic linear scan voltammograms of the first reduction step of the 


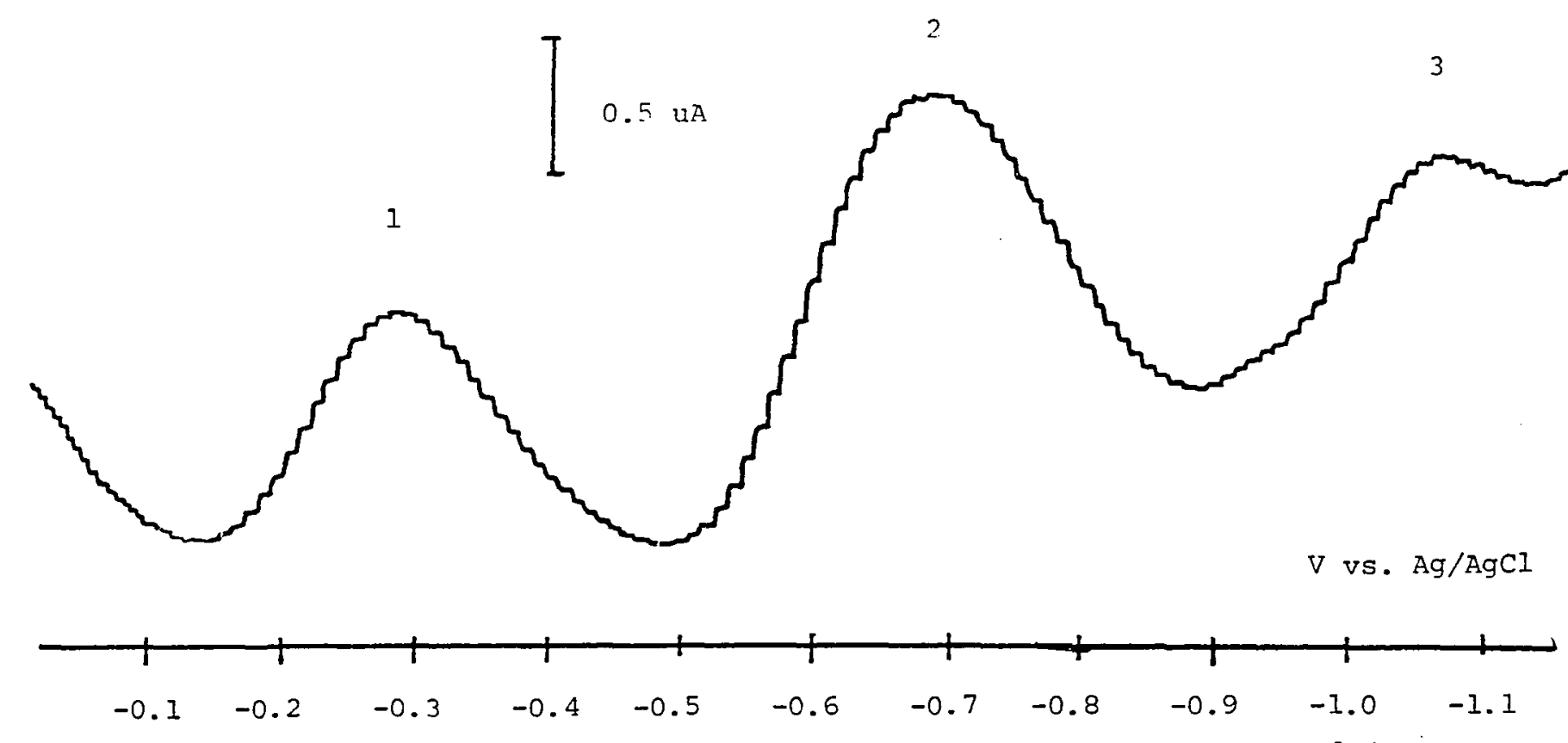

Figure 2. Differential pulse polarogram of $\mathrm{As}(\mathrm{V})$ in $0.5 \mathrm{M}$ catechol, $\mathrm{pH}=1.0$, $1.25 \mathrm{mM}$ As ( $\mathrm{V})$. Dropping Mercury Electrode. Scan rate $=10 \mathrm{mV} / \mathrm{s} .100 \mathrm{mV}$ pulse. $0.1 \mathrm{~N} \mathrm{iClO}_{4}$ electrolyte. 


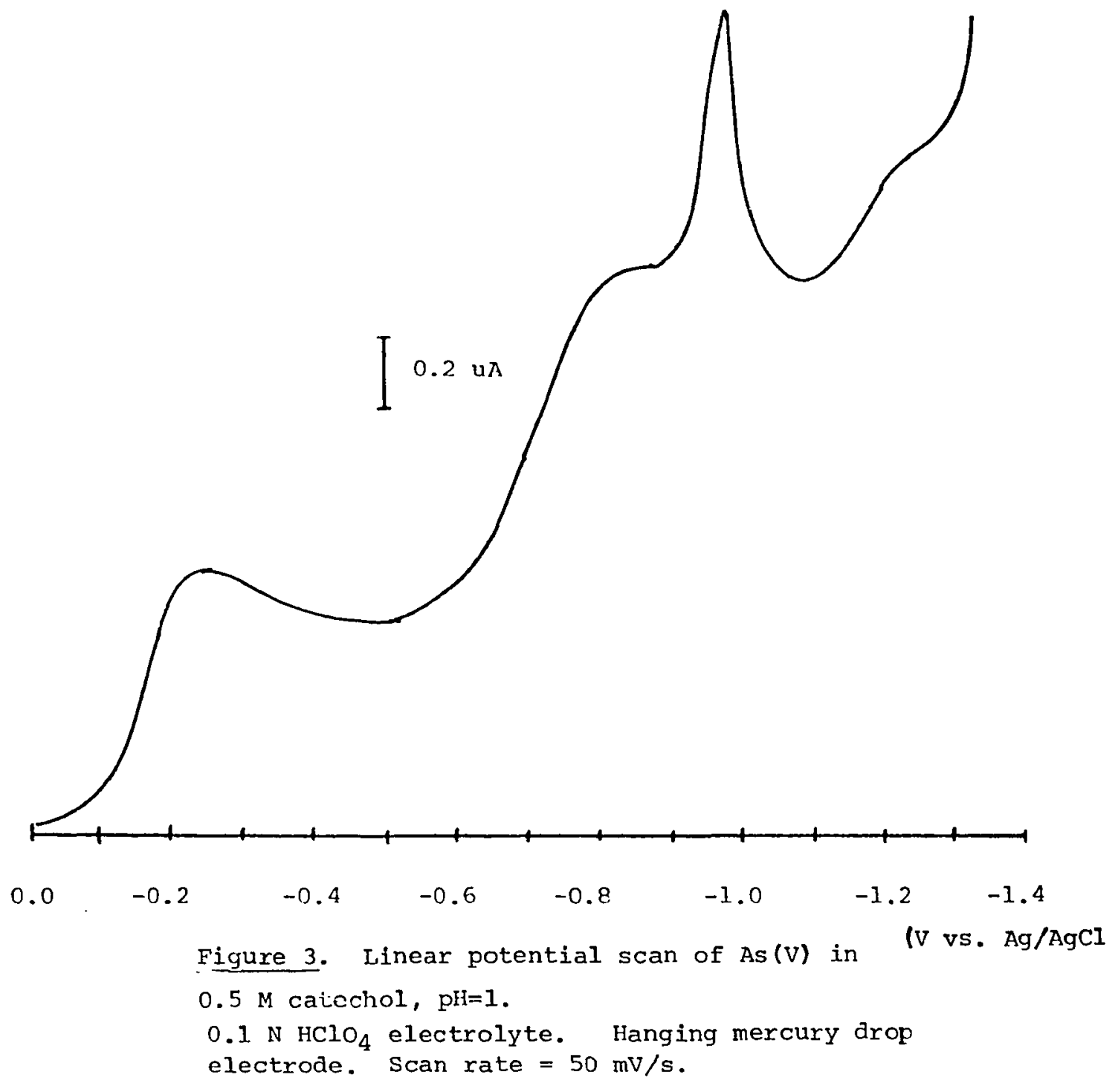




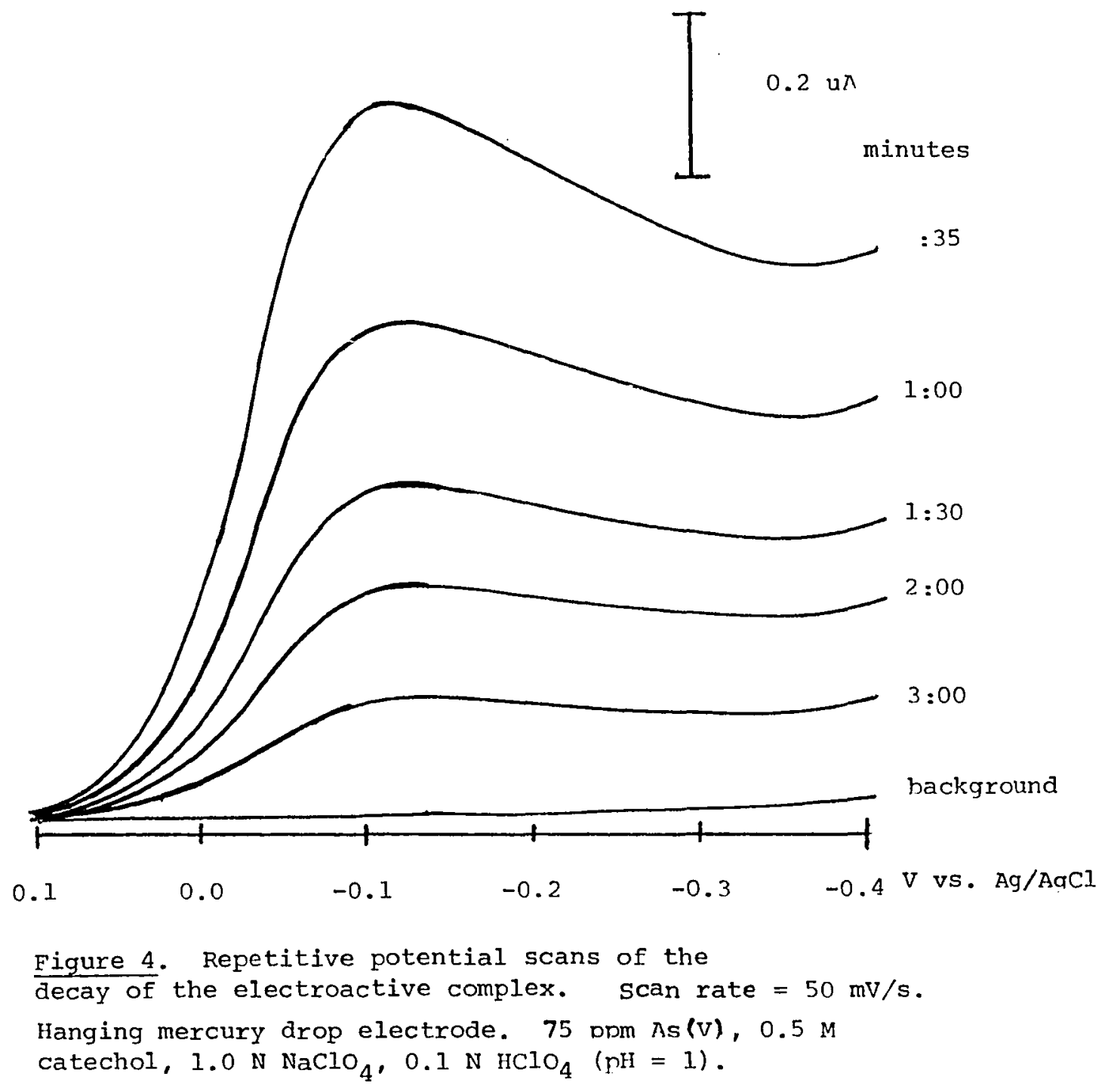


EAC when reduced at a hanging mercury drop electrode (HMDE). The decrease of the wave height as the reaction progresses illustrates the decay of the electroactive complex. The CV curves were measured and the data representing the variation of current as a function of time was tested by the appropriate kinetic model. 
Linear scan voltammetry, (LSV), is a very powerful technique for the study of electrode mechanisms. Diagnostic criteria were developed by Nicholson and Shain (52) to determine the type of heterogeneous electrode mechanisms occuring during electrode reaction. Information about the chemical and physical characteristics of an electrode reaction can be determined from current voltage curves. The change in current value, peak shape, and other parameters with the variation of experimental conditions; such as scan rate, provide criteria useful in determining electrode mechanisms.

Cyclic scan voltammetry was used to determine whether the reduction of the $A s(V)$-catechol electroactive complex was a reversible electron transfer. In Figure 5 a cyclic voltamogram of the As(V)catechol electroactive complex is presented. It can be seen from this illustration that there is no evidence of an anodic peak recorded during the return portion of the cyclic scan. The reduction of the As(V)catechol electroactive complex is a completly irreversible reaction, as the cyclic scan voltammetry results demonstrate. The reduction of As(III) is also completely irreversible under these conditions. Linear scan voltammetry or cyclic scan voltammetry may be used to evaluate the characteristics of the electrode reaction.

The shape of the reduction peak in linear scan voltammetry is a very important diagnostic criteria (52). In a simple electron transfer the current rises when the reduction potential is approached, reaches a peak value as the scan continues and decays as the concentration of the reacting species becomes depleted in the area around the electrode. When the reduction mechanism involves additional steps besides electron 


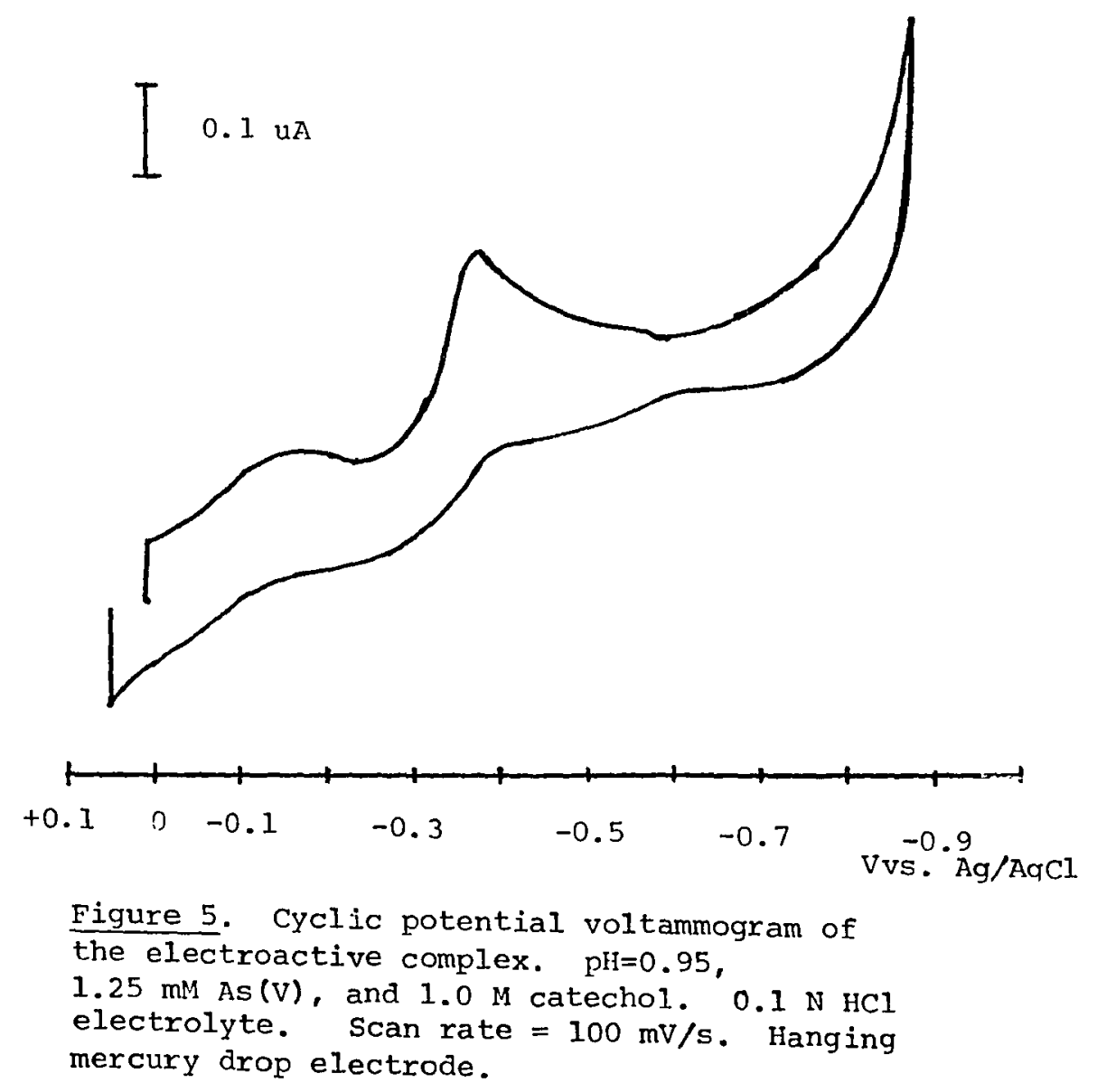


transfer the shape of the reduction peak changes. The reduction peak can take on a wave or s-shaped appearance with a current plateau following the current maximum rather than the current decay observed for a simple reduction. The shape of the reduction peak for the EAC reduction is influenced by a number of factors; catechol concentration, $\mathrm{pH}$, and the progress of reaction. Information about the electrode reaction mechanism can be gained by performing linear potential scans of the As(V)-catechol system as the electroanalytical parameter of scan rate varied for each recording.

The experiments conducted to study the reaction mechanism of the As(V)-catechol electroactive complex were performed in the manner described below. A reaction solution was prepared which contained supporting electrolyte, $1.0 \mathrm{M} \mathrm{HCl}$, and sufficient catechol to produce solutions with catechol concentrations of $0.1 \mathrm{M}$ to $1.3 \mathrm{M}$. These solutions were well mixed and deaerated by nitrogen gas. A series of linear scan voltammograms were recorded for each reaction solution. The scans commenced at $-0.1 \mathrm{~V}$ and continued to $-0.4 \mathrm{~V}$ in a cathodic scan. The scan then reversed direction and returned to the initial potential. Four different scan rates were used to record the cyclic voltammograms, 10, 50, 100 , and $200 \mathrm{mV} / \mathrm{s}$. Arsenate was then added to the reaction mixture as an aliquot of $A s(V)$ stock solution to yield a concentration of $1.25 \mathrm{~mm}, 94 \mathrm{ppm}$. The kinetic reaction was timed as described previously and the solution was deaerated and mixed for 30 seconds. Following mixing a quartet of cyclic scan voltammograms were recorded beginning with the scan rate of $200 \mathrm{mV} / \mathrm{s}$ and finally recording the 10 $\mathrm{mV} / \mathrm{s}$ scan. This quartet of voltammograms was repeatedly recorded until 
the reaction mixture had reached equilibrium. In Figure 6 a series of these quartet scans are shown. From one reaction mixture the kinetic data can be plotted for four different scan rates. The change in reduction peak shape can be observed at different scan rates. 


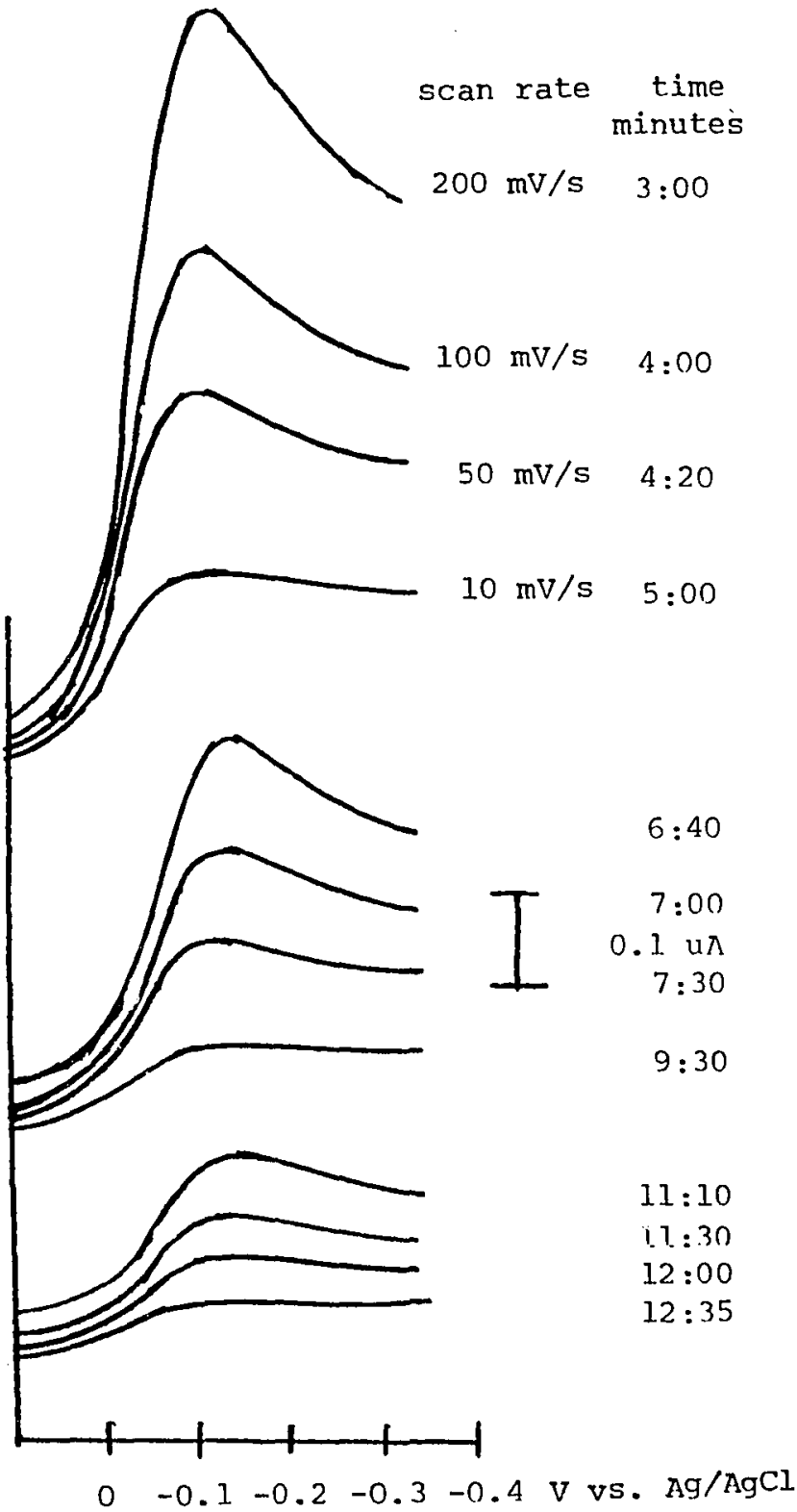

Figure 6. Linear potential scans of the decay of the electroactive complex at scan rates of: $200 \mathrm{mV} / \mathrm{s}, 100 \mathrm{mV} / \mathrm{s}, 50 \mathrm{mV} / \mathrm{s}$, and $10 \mathrm{mV} / \mathrm{s}$. $\mathrm{pH}=1.0,1.25 \mathrm{mM} \mathrm{As}(\mathrm{V})$, and $0.5 \mathrm{M}$ catechol. $0.1 \mathrm{~N} \mathrm{NaClO}_{4}$ and $0.1 \mathrm{~N} \mathrm{HClO}_{4}$ electrolyte. Hanging mercury drop electrode. 
DIFFERENTIAL PULSE POLAROGRAPHIC STUDIES OF As(III) and As(V) IN THE PRESENCE OF CATECHOL

The technique of differential pulse polarography (DPP) is well known as a very sensitive method for trace analysis with reported detection limits in the 1 - $100 \mathrm{nM}$ range. Myer and Osteryoung (33) have developed a procedure for quantifying the concentration of As(III) ion in water, sewage, and sludge samples. The technique of differential pulse polarography was used for analysis and the calculated detection limits of $1 \mathrm{nM}, 0.3 \mathrm{ppb}$, were reported by Myer and Osteryoung (28) for As(III) analysis. The great sensitivity of this method suggested that the concentration of As(V) could be determined by DPP after it was complexed with catechol and formed the electroactive complex. Differential pulse polarography experiments were conducted to assess the feasibility of analyzing for As(V). The method developed by Myer and Osteryoung (28) was used to analyze solutions of As(III), As(III)catechol, and As(V)-catechol.

Experiments were conducted to answer the following questions. 1. What is the effect of catechol on the polarography of As(III)? 2. Can polarographic methods be used to analyze for As(V) in aqueous solutions?

The method of Myer and Osteryoung (28) involves treating the water sample with hydrochloric acid to produce a $1.0 \mathrm{M}$ solution. The differential pulse polarogram is recorded by scanning the potential range of $-0.240 \mathrm{~V}$ to $-1.0 \mathrm{~V}$ at a rate of $5 \mathrm{mV} / \mathrm{s}$. The pulse height was $100 \mathrm{mV}$ and applied once every second. The drop time was also one second. A DPP recording of the reduction of As(III) in $0.5 \mathrm{M}$ catechol solution to the elemental state, $A s(0)$, and then to arsine, AsH, 
contains two peaks, as can be seen in Figure 7 . The peak for the first reduction step, As(III) $\rightarrow A s(0)$, is observed at approximately $-0.65 \mathrm{~V}$ versus the $\mathrm{Ag} / \mathrm{AgCl}$ reference electrode. A calibration curve for $\mathrm{As}$ (III) in catechol was prepared by plotting the values of peak current versus arsenite concentration. A linear range of $0.3 \mathrm{ppb}$ to $60 \mathrm{ppm}$ was reported by Myer and Osteryoung (28).

Similar experiments were conducted on As(III) solutions which contained catecho1. The purpose of these experiments was to observe the effect of catechol on the reduction of As(III), to prepare a calibration curve, and determine detection limits for As(III) in a catechol solution.

The water samples were prepared by using $10.0 \mathrm{ml}$ of $1.0 \mathrm{M} \mathrm{HClO}$ in distilled water and an addition of $0.55 \mathrm{~g}$ of sublimed catechol. This solution shall be refered to as the background solution. Differential pulse polarographic curves were recorded for the background solution. An aliquot of As(III) was then added to the background solution to produce the desired concentration of $\mathrm{As}$ (III) in the test sample. A differential pulse polarogram was then recorded for the As(III)-catechol solution. The As(III) concentration was increased by another standard addition of stock solution and a curve was again recorded. This procedure was repeated until a range of As(III) concentrations had been evaluated. In Figure 8 we see a series of such differential pulse polarograms of As(III) solutions. A calibration curve was then prepared by plotting the peak current ws. As(III) concentration for solutions of $0.5 \mathrm{M}$ catechol.

To prepare a calibration curve for analysis of As(V) solutions a 


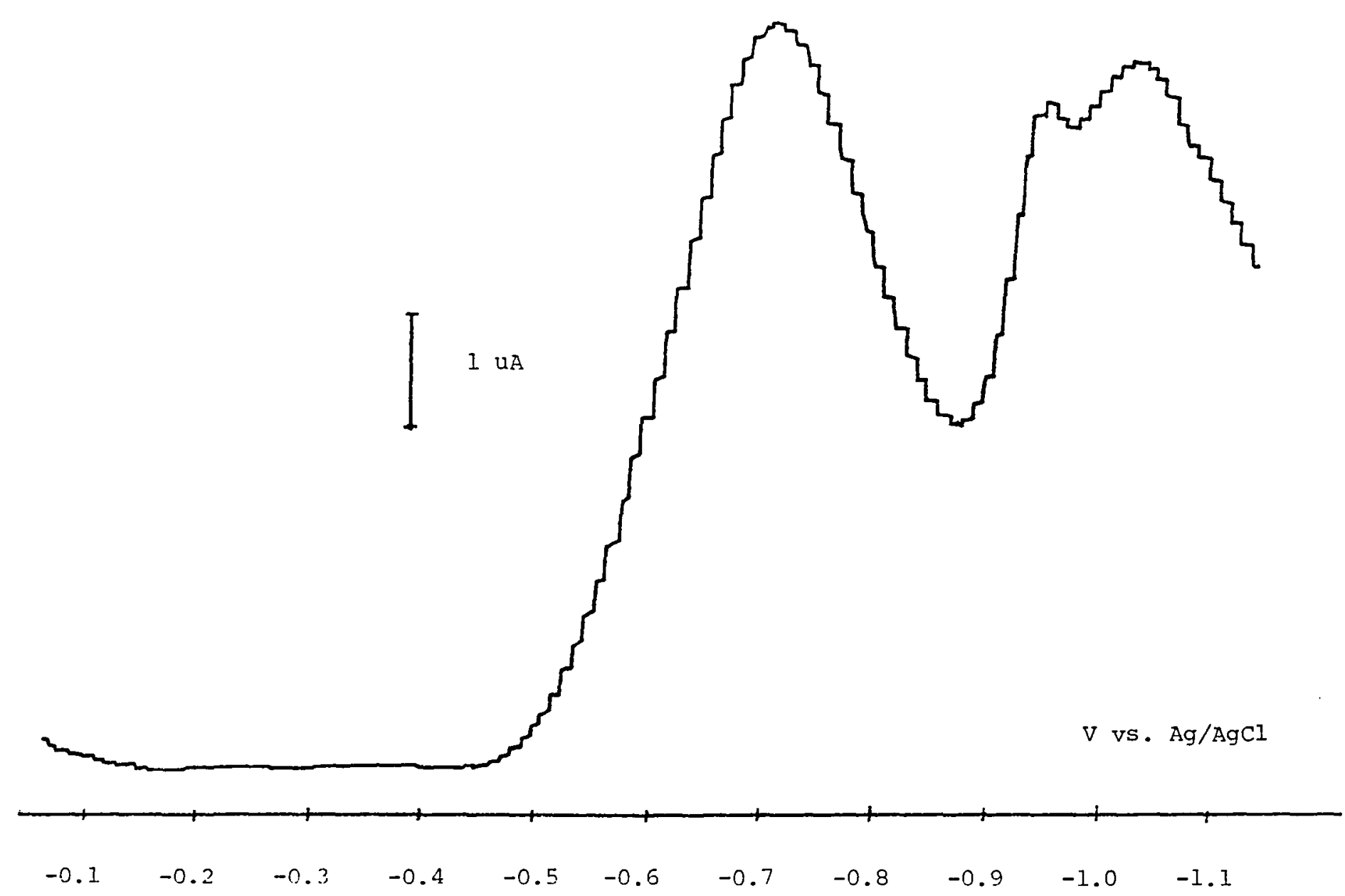

Figure 7. Differential pulse polarogram of As(III). in '. $\mathrm{M}$ HCIO4, $0.5 \mathrm{M}$ catechol anc. $3 \mathrm{ppm} \mathrm{As}$ (III).

Dropping mercury electrode, drop time $=1 \mathrm{~s}, 100 \mathrm{mV}$ pulse, scan rate $=10 \mathrm{mV} / \mathrm{s}$ 


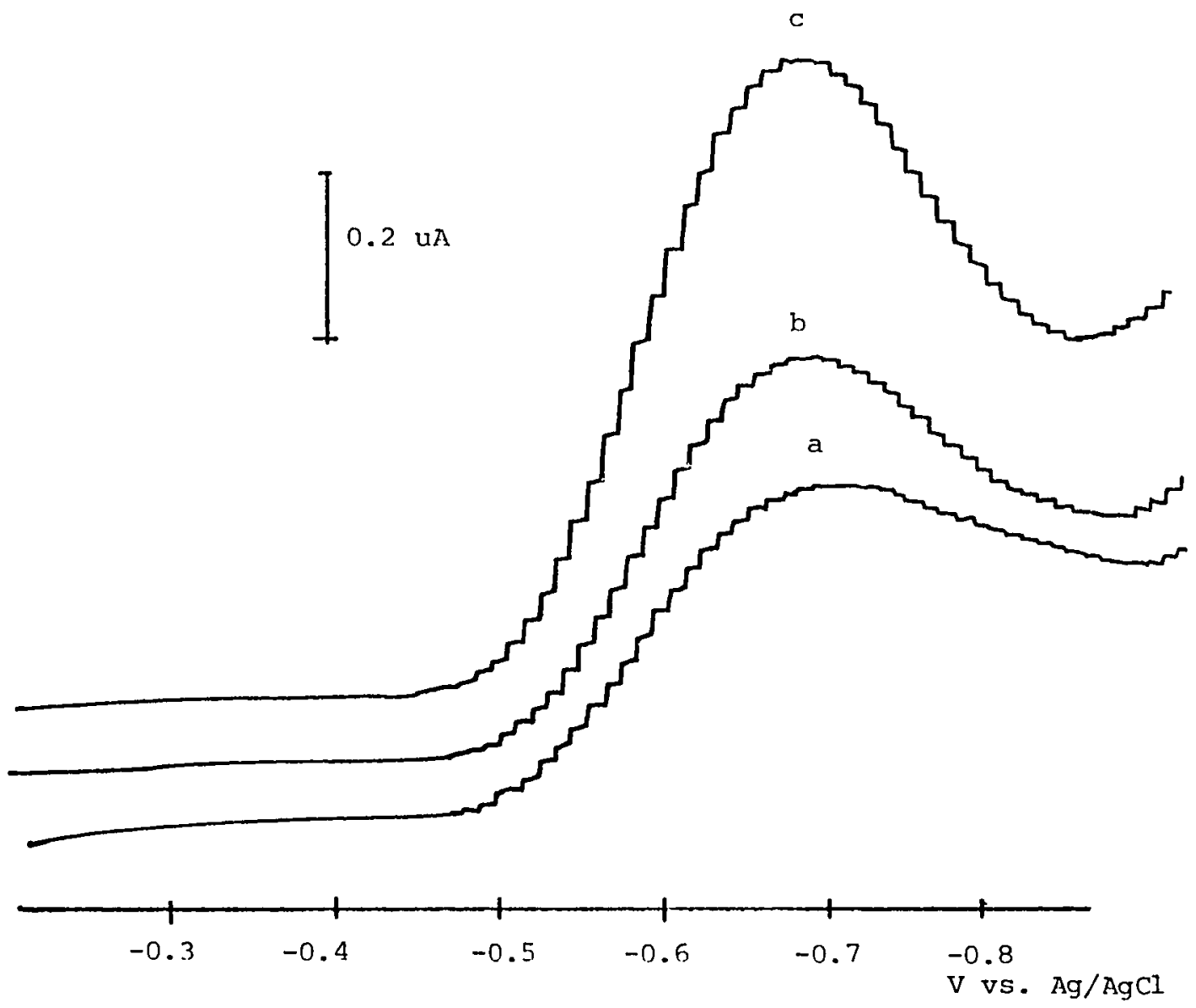

Figure 8. Differential pulse polarograms of a series of $A s$ (III) solutions with $0.5 \mathrm{M}$ catechol a. $284 \mathrm{ppb} \mathrm{As}$ (III)

b. $342 \mathrm{ppb}$ As (III)

c. $542 \mathrm{ppb}$ As (III)

$0.1 \mathrm{~N} \mathrm{HCl}$ and $0.9 \mathrm{~N} \mathrm{NaCl}$ electrolyte scan rate $=10 \mathrm{mV} / \mathrm{s}$, pulse height $=50 \mathrm{mV}$ dropping mercury electrode = working electrode. 
single kinetic experiment must be conducted for each As(V) concentration to be included in the concentration range. The reaction solution was prepared to contain $0.1 \mathrm{~N} \mathrm{HCl}$ and $0.5 \mathrm{M}$ catechol. A DPP scan was performed on this solution and the resultant CV curves will be referred to as the background curves. The applied potential was then switched to $-0.4 \mathrm{~V}$, a potential which lies between the first two reduction peaks of the electroactive complex and the current was recorded as a function of time. Then As(V) was added to the background solution as an aliquot of arsenate stock solution to produce the desired final As(V) concentration. The current produced by the EAC is then recorded on the strip chart recorder at the same potential. The current produced from reduction of the electroactive complex is directly proportional to the concentration of the arsenate ion. In Figure 9 a copy of such a strip chart recording is presented. The working electrode was a dropping mercury electrode. The drop time was 1 second. The current oscillations due to each falling mercury drop are not observed because the operating mode chosen, (sampled DC) only samples the current after the drop is formed. This current is measured just once for each drop's lifetime and is then presented to the strip chart recorder as a constant value each second. The strip chart recording displays the exponential decay of the electroactive complex. The current at any point in time was measured on the decay trace from an extension of the background current baseline. A concentration range of $1 \mathrm{ppm}$ to $18 \mathrm{ppm}$ was examined. The data present on the strip chart recordings was used to prepare a calibration curve for $A s(V)$ and to determine the detection limit. 


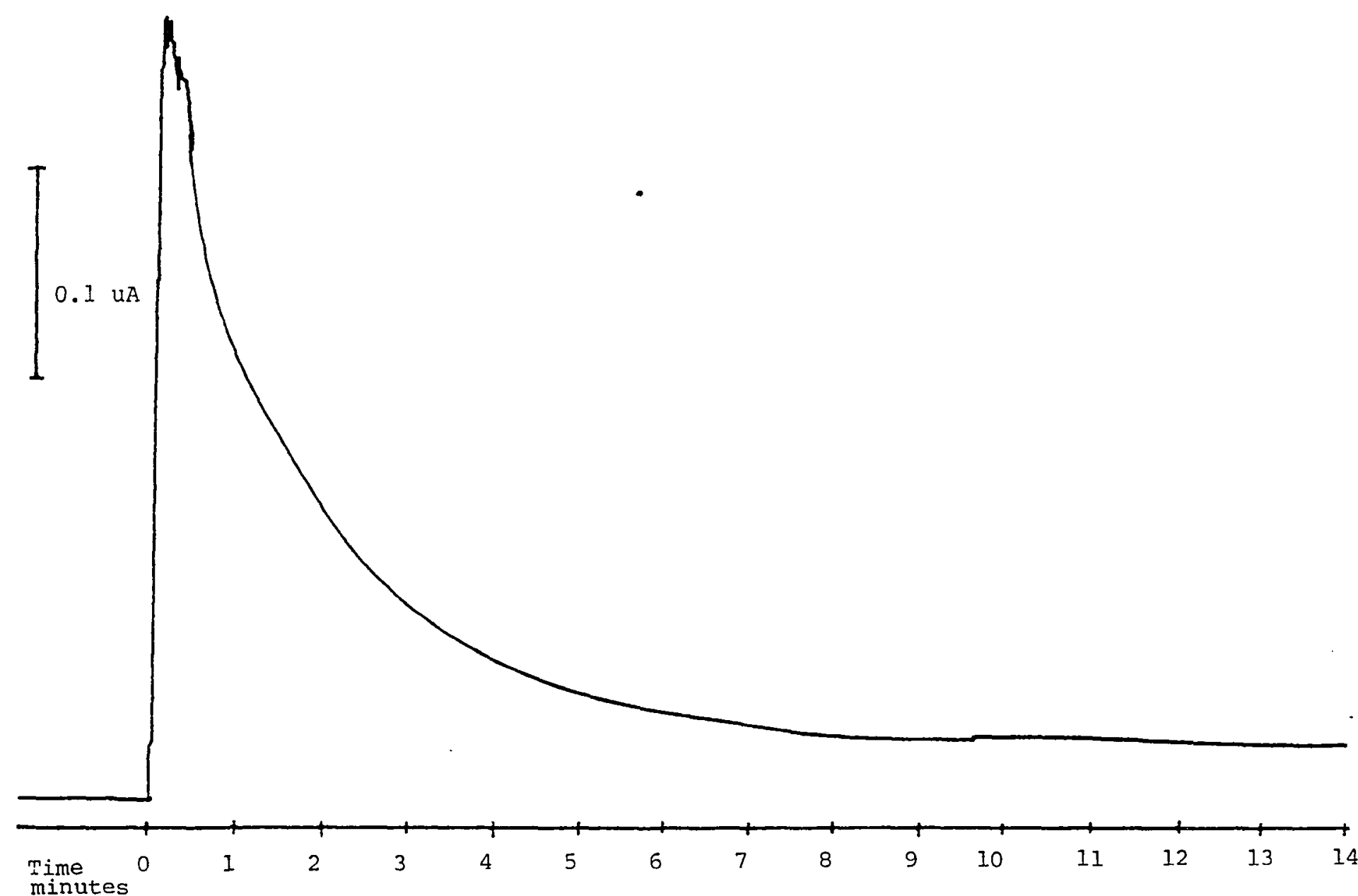

Figure 9. Reduction current for the decay of the EAC at applied potential of $-0.4 \mathrm{~V} \mathrm{vs} . \mathrm{Ag} / \mathrm{AgCl}$ $1.0 \mathrm{H} \mathrm{HCl}, 0.5 \mathrm{M}$ catechol, and $45 \mathrm{ppm} \mathrm{As}(\mathrm{V})$.

Dropping mercury electrode, polarographic analysis in sampled DC mode. 
STRIPPING VOLTAMMETRY

The anodic stripping method developed by Davis et al. (40) for analysis of As(III) is performed at gold or platinum electrodes in acidic solutions. The As(III) is reduced to the elemental state in the deposition step and collected at the working electrode. In the stripping step the deposited arsenic is oxidized producing a current peak used as the analytical signal. Preliminary experiments were conducted to evaluate the possibility of applying anodic stripping voltammetry to the analysis of $A s(V)$-catechol solutions.

The anodic stripping experiments were conducted at a gold rotating disc electrode operated at a rotation rate of $1000 \mathrm{rpm}$. The analytical solution conditions were $1.0 \mathrm{NHC104}$ and $3.8 \mathrm{ppm} \mathrm{As(III)}$ as described by Davis et al. (40). A deposition potential of $-0.4 \mathrm{~V}$ was applied for 2 minutes followed by an anodic scan to oxidize the arsenic deposit and produce the analytical signal. Solid catechol was then added to the analytical solution to produce a $0.5 \mathrm{M}$ catechol concentration. The anodic stripping experiment was again performed, and no stripping peak was observed. Further experiments were not conducted.

The cathodic stripping method developed by Holak (42) was examined next for possible application to arsenic speciation. This technique requires the addition of $\mathrm{Se}(\mathrm{IV})$ to the analytical solution.

The method of Holak (42) was performed on As(III) solutions containing $1.0 \mathrm{~N} \mathrm{HC10}$ and $50 \mathrm{ppb}$ of Se(IV) at a HMDE. After successful 4

use of Holak's method, solid catechol was added to the analytical solution to produce a $0.5 \mathrm{M}$ catechol concentration. A cathodic 
stripping peak was not observed for As(IIL)-catechol solutions. Additional experiments were conducted on selenium (IV) solutions with and without catechol. Linear scan voltammetry demonstrated that the Se(IV) reduction peak is shifted in the cathodic direction by the addition of catechol. The shift of the selenium reduction peak by catechol apparently prevented successful deposition of As(III) at the mercury electrode and the cathodic stripping of As(III) failed to occur. Consequently this method was abandoned.

Two very similar methods were developed by Henze, Joshi, and Neeb (43), and Sadana (44) for the cathodic stripping of As(III). These techniques involve deposition of As(III) into a HMDE from an acidified aqueous solution containing $\mathrm{Cu}(\mathrm{II})$. The arsenic deposit is stripped by a cathodic scan to produce a large current peak suitable for quantification. The $\mathrm{Cu}(\mathrm{II})$ ion is apparently reduced at the HMDE and forms an intermetallic arsenic-copper compound in the working electrode. Preliminary experiments were performed to test the possibility of applying the methods of Henze et al. (43) and of Sadana (44) to the determination of $A s(I I I)$ and $A s(V)$ concentrations in solutions containing catechol.

The methods of Henze, et al. (43) and Sadana (44) differ in a few features, most importantly, the copper (II) concentration. Henze, et a1. (43) recommend a $\mathrm{Cu}(\mathrm{II})$ concentration of $2 \mathrm{mM}$ or $127 \mathrm{ppm}$. Sadana (44) recommends 79 uM Cu(-II), 5 ppm, for stripping analysis. In Table I the recommended solution conditions and instrumental parameters are summarized for each method. Deposition potential, deposition time, analytical ranges, and acid concentration are all very similar for both 
methods.

TABLE I

EXPERIMENTAL CONDITIONS FOR CATHODIC STRIPPING VOLTAMMETRY WITH COPPER CODEPOSITION

$\begin{array}{lll} & \text { HENZE et al. (43) } & \text { SADANA (44) } \\ \text { [Cu(II)] } & 2 \mathrm{mM} & \begin{array}{l}79 \mathrm{uM} \\ 127 \mathrm{ppm}\end{array} \\ \text { acid } & 1.0 \mathrm{~N} \mathrm{HCl} & 0.75 \mathrm{~N} \mathrm{HCl} \\ \text { dep.pot. } & -0.55 \mathrm{~V} & -0.6 \mathrm{~V} \\ \text { dep. time } & 1 \mathrm{~min} . & 2 \mathrm{~min} \\ \text { peak.pot. } & -0.77 \mathrm{~V} & -0.72 \mathrm{~V} \\ \text { scan mode } & \text { DPV } & \text { DPV } \\ \text { pulse } & 0.050 \mathrm{~V} & 0.075 \mathrm{~V} \\ \text { height } & & \text { HMDE } \\ \text { electrode } & \text { HMDE } & 0 \mathrm{to} 20 \mathrm{ppb} \\ \text { anal.rg. } & 0.2 \text { to } 20 \mathrm{ppb} & 1 \mathrm{ppb}\end{array}$

Experiments were conducted following both procedures. The technique of Henze, et al. (43) was tested with a solution of $1.0 \mathrm{~N} \mathrm{HCl}$, $25.3 \mathrm{uM}, 19 \mathrm{ppm} \mathrm{As}$ (III), and $2 \mathrm{mM}, 127 \mathrm{ppm} \mathrm{Cu(II).} \mathrm{A} \mathrm{large} \mathrm{"stripping"}$ peak was observed in the region of $-0.79 \mathrm{~V}$ versus the $\mathrm{Ag} / \mathrm{AgCl}$ reference electrode. After sufficient solid catechol had been added to the test solution to produce a $0.5 \mathrm{M}$ solution the stripping peak was significantly reduced.

The technique of Sadana (44) was evaluated in a similar manner. The supporting electrolyte contained $1.0 \mathrm{~N} \mathrm{HCl}$. This solution was 
deaerated and a differential pulse voltametry (DPV) scan was recorded following a 2 minute deposition at $-0.6 \mathrm{~V}$. An aliquot of $\mathrm{Cu}(\mathrm{II})$ stock solution was added to the electrolyte resulting in a concentration of $0.1 \mathrm{mM},(6.4 \mathrm{ppm})$. A DPV scan was recorded, which can be seen in Figure 10, curve a. Then an aliquot of As(III) stock solution was added to the mixture producing a concentration of $0.15 \mathrm{uM}$, (11ppb). This solution was again analyzed by DPV, as seen in Figure 10, curve b. Finally sufficient solid catechol was added to the analytical solution to produce a concentration of $0.5 \mathrm{M}$. Another DPV was recorded of the solution and presented in Figure 10, curve c. It can be seen that the $\mathrm{HCl} / \mathrm{Cu}(\mathrm{II})$ solution, Figure 10, curve a, did not produce a stripping peak. The addition of As(III) to the solution produced a dramatic stripping peak at $-0.72 \mathrm{~V}$ versus the $\mathrm{Ag} / \mathrm{AgCl}$ reference electrode. After addition of catechol the peak current is much reduced, it no longer has a good shape and the peak potential has shifted to $-0.76 \mathrm{~V}$. Very similair results were obtained using the method developed by Henze, et a1. (43).

The results with As(III) were sufficiently encouraging to warrant conducting an experiment with $A s(V)$. The method of Sadana was used for these experiments. Two sets of data were collected for a single As(V) kinetic experiment. The background solution was composed of $1.0 \mathrm{~N}$ HCl and $79 \mathrm{uM}, 5 \mathrm{ppm} \mathrm{Cu}$ (II). In Figure 11 and Figure 12 a DPV scan of this background solution is shown for experiments conducted at two different deposition potentials, $-0.3 \mathrm{~V}$ and $-0.5 \mathrm{~V}$ respectively. Then an aliquot of As(V) stock solution was added to the background solution to produce an arsenic concentration of $2.3 \mathrm{mM},(175 \mathrm{ppm})$. The solution was mixed 

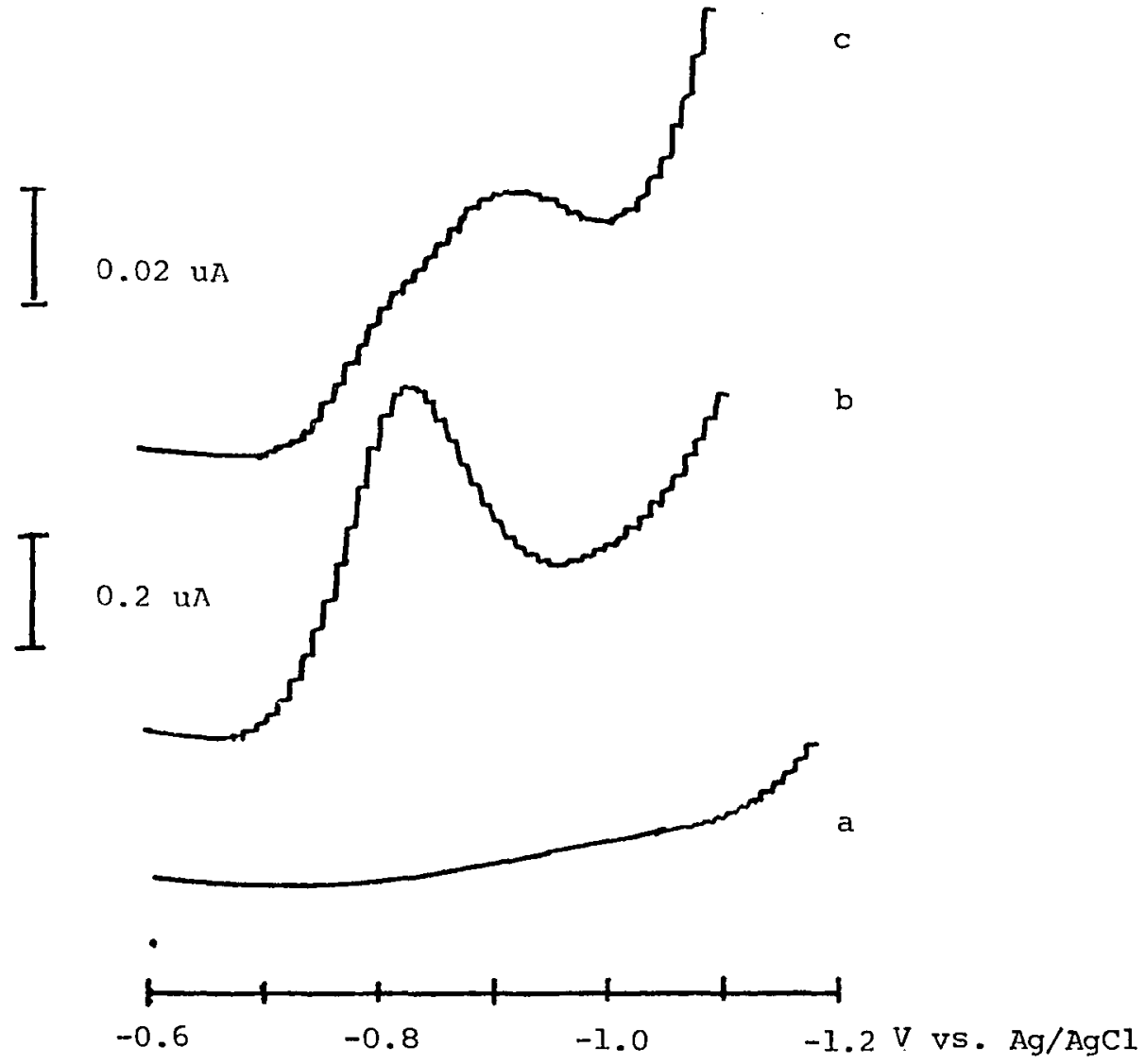

Figure 10. Differential pulse cathodic stripping of As (III) in $0.5 \mathrm{M}$ catechol. a. $1.0 \mathrm{~N} \mathrm{HCl}$ and $6.4 \mathrm{ppm} \mathrm{Cu}$ (II)

b. $1.0 \mathrm{~N} \mathrm{HCl}, 6.4 \mathrm{ppm} \mathrm{Cu}$ (II), $11 \mathrm{ppb} \mathrm{ns}$ (III) c. $1.0 \mathrm{~N} \mathrm{HCl}, 6.4 \mathrm{ppm} \mathrm{Cu}$ (II). $11 \mathrm{ppb} \mathrm{As}$ (III) anà $0.5 \mathrm{M}$ catechol. denogition potential $=-.06 \mathrm{~V}$ deposition feriod $=2 \mathrm{~min}$ stir $/ 0.5 \mathrm{~min}$ qulet scan rate $=5 \mathrm{mV} / \mathrm{s}$, pulse helght $=50 \mathrm{mV}$ Hanging mercury drop electrode. 


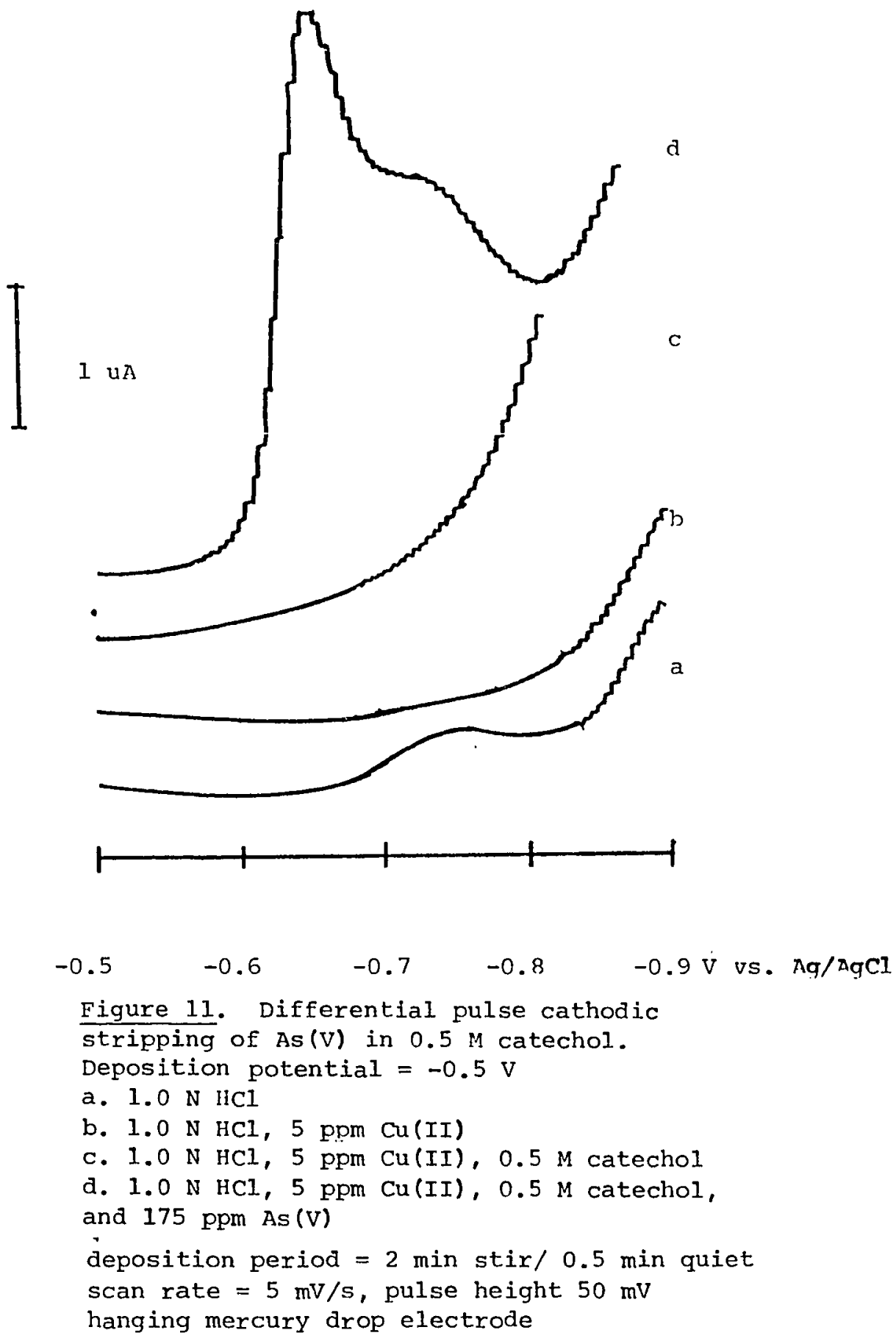




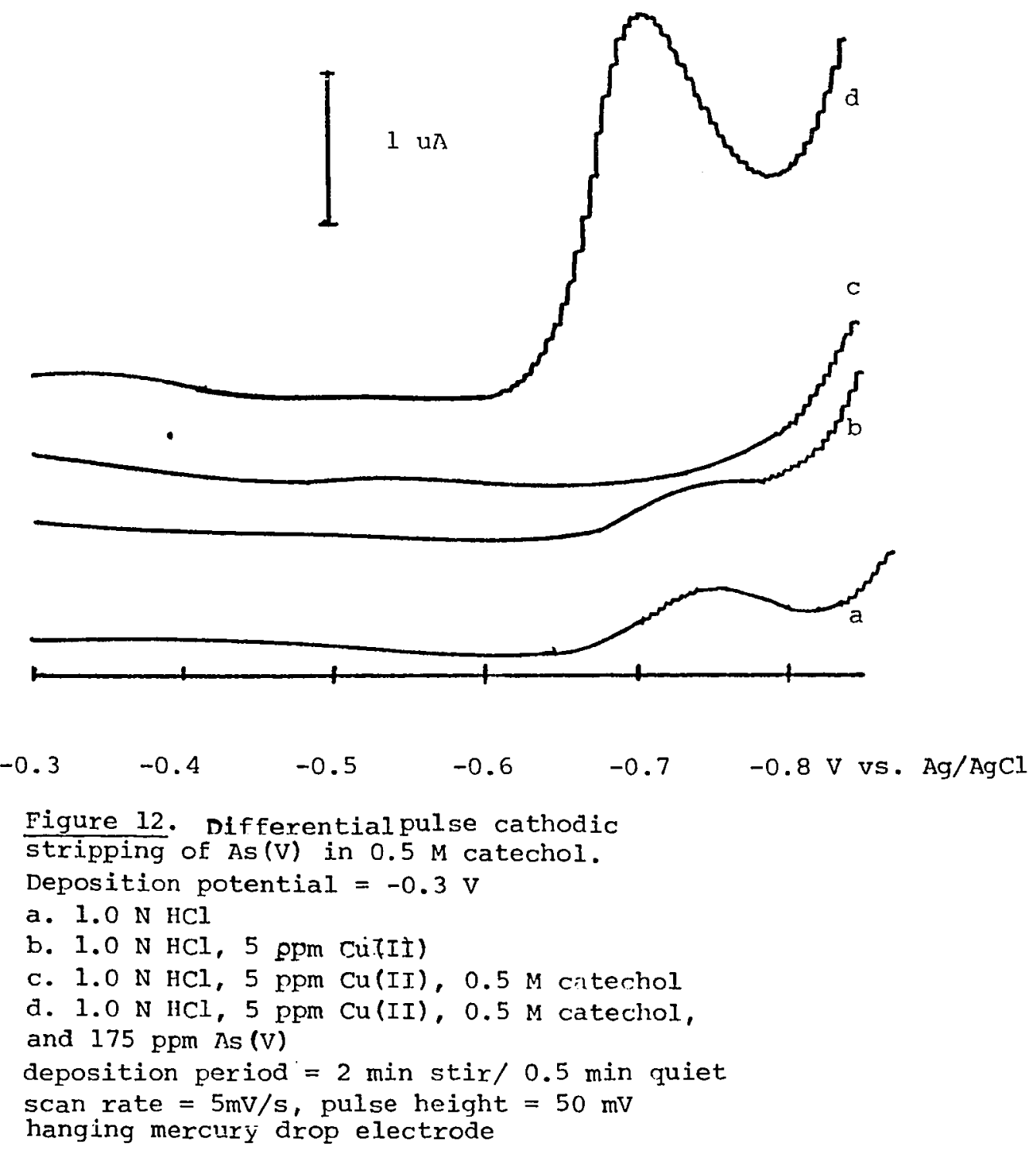


and differential pulse cathodic stripping scans were recorded as the EAC decayed. The cathodic stripping voltammograms were conducted alternately at $-0.3 \mathrm{~V}$ and $-0.5 \mathrm{~V}$ deposition potentials. In Figures 11 and 12 two examples of DPV scans are shown. When the deposition potential equals $-0.3 \mathrm{~V}$ a single peak is observed in the stripping experiment. A double stripping peak is observed when the deposition potential is equal to $-0.5 \mathrm{~V}$. This curious double peak may be caused by the large concentration of arsenic deposited into the working electrode. The results of these experiments were sufficiently encouraging to suggest further study in the application of copper aided cathodic stripping voltammetry to analysis of As(V) concentrations. Consequently, a number of additional experiments were planned. 


\section{Enhancement}

The cathodic stripping methods developed by Henze et al. (43) and Sadana (44) possess a number of unusual characteristics. The most intriguing aspect is the unexpectedly large stripping peak observed during analysis of solutions containing As(III) at the parts per billion leve1. This phenomena was not investigated by either research group. The enhanced stripping current peak suggests that low levels of As(V), complexed by catechol, could also be successfully measured by cathodic stripping voltammetry. Experiments were conducted to determine the source of the enhanced stripping peak. These experiments are referred to as coulonetric studies.

The coulometric studies involved integrating the current-voltage curves recorded for reduction of As(III)-Cu(II) solutions to determine the number of coulombs consumed in the electrode reaction. The number of coulombs can then be converted into moles of copper or arsenic reduced at the electrode by use of Faraday's Law. The number of coulombs consumed in reduction reactions were measured with the current integrator module of a Heka potentiostat. The current could be integrated during a voltage scan and the resultant values of charge displayed on a $x-y$ recorder as a plot of coulombs versus applied potential.

In Figure 13 a number of current-voltage curves are shown for linear scan voltammograms performed at a hanging mercury drop electrode. The linear potentials scans seen in Figure 13 were conducted at a scan rate of $50 \mathrm{mV} / \mathrm{s}$, initial potential of +0.052 and a final potential of $0.998 \mathrm{~V}$ versus the $\mathrm{Ag} / \mathrm{AgCl}$ reference electrode. Curve (a) represents 


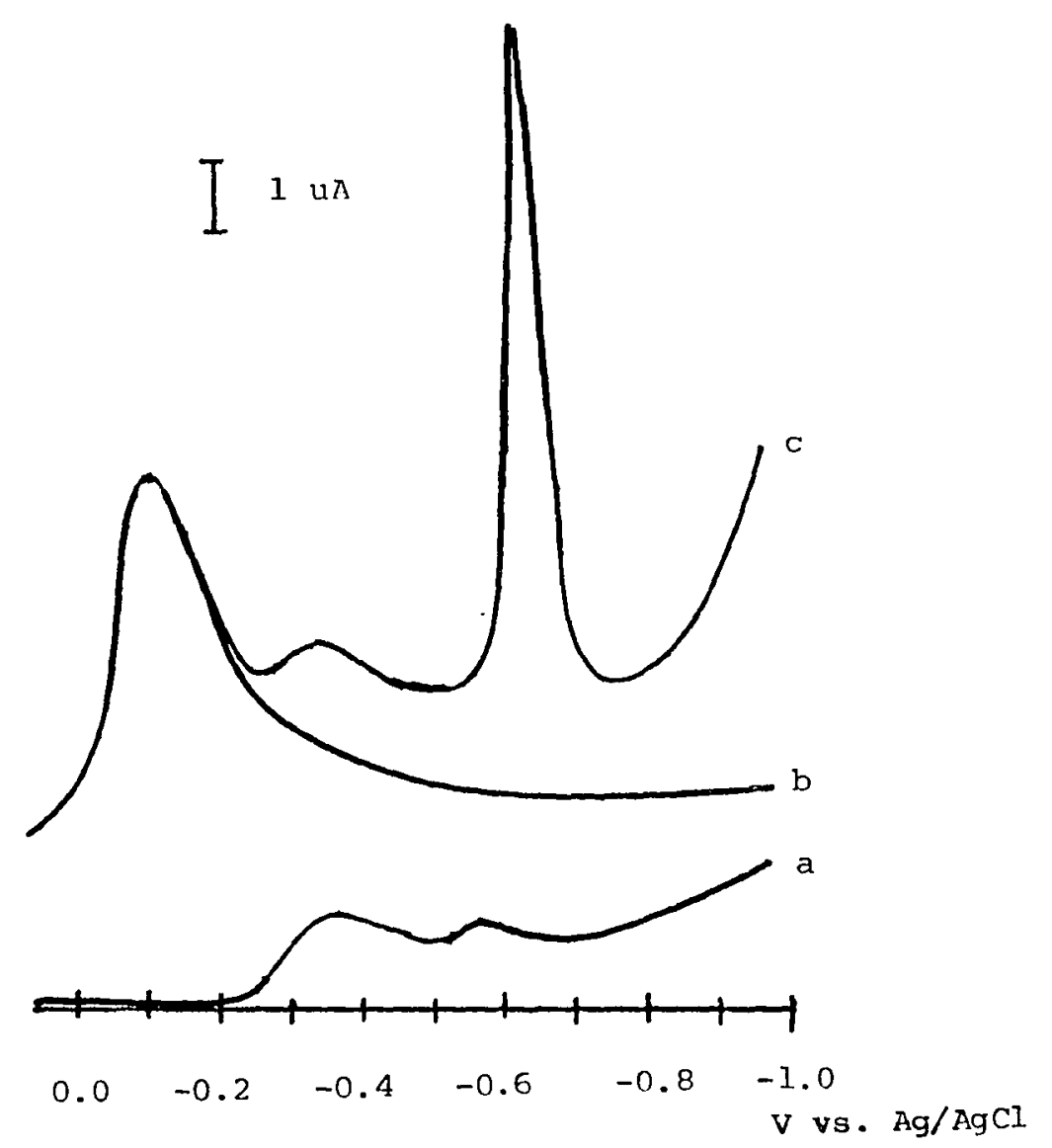

Figure 13. Current voltage curves for integration studies.

a. $1.0 \mathrm{~N} \mathrm{HCl}, 250$ uM As(III)

b. $1.0 \mathrm{~N} \mathrm{HCl}, 2 \mathrm{mM} \mathrm{Cu}$ (II)

c. $1.0 \mathrm{~N} \mathrm{HCl}, 250$ uM As(III), $2 \mathrm{mM} \mathrm{Cu}$ (II) scan rate $100 \mathrm{mV} / \mathrm{s}$

Linear scan voltammetry at hanging mercury ảrop electrode. 
the $\mathrm{CV}$ curve of the supporting electrolyte, $1.0 \mathrm{~N} \mathrm{HCl}$ and an aliquot of As(III) to produce a solution of $0.25 \mathrm{mM}$ or $19 \mathrm{ppm}$. A similair solution of electrolyte and $127 \mathrm{ppm} \mathrm{Cu(II)} \mathrm{is} \mathrm{shown} \mathrm{in} \mathrm{curve} \mathrm{(b).} \mathrm{In} \mathrm{curve} \mathrm{(c),}$ a CV scan was recorded of a solution containing $19 \mathrm{ppm} \mathrm{As}$ (III) and $2 \mathrm{mM}$, $127 \mathrm{ppm} \mathrm{Cu}(\mathrm{II})$. The linear scan voltammogram shows an enhanced "stripping" peak attributable to reduction of the arsenic-copper intermetallic. In Figure 14 the integration curves are shown for solutions of $A s$ (III) alone, $\mathrm{Cu}$ (II) alone and a mixture of As(III) and $\mathrm{Cu}(\mathrm{II})$. From this data the number of coulombs for solutions of arsenic, copper, and arsenic and copper can be determined. 
48

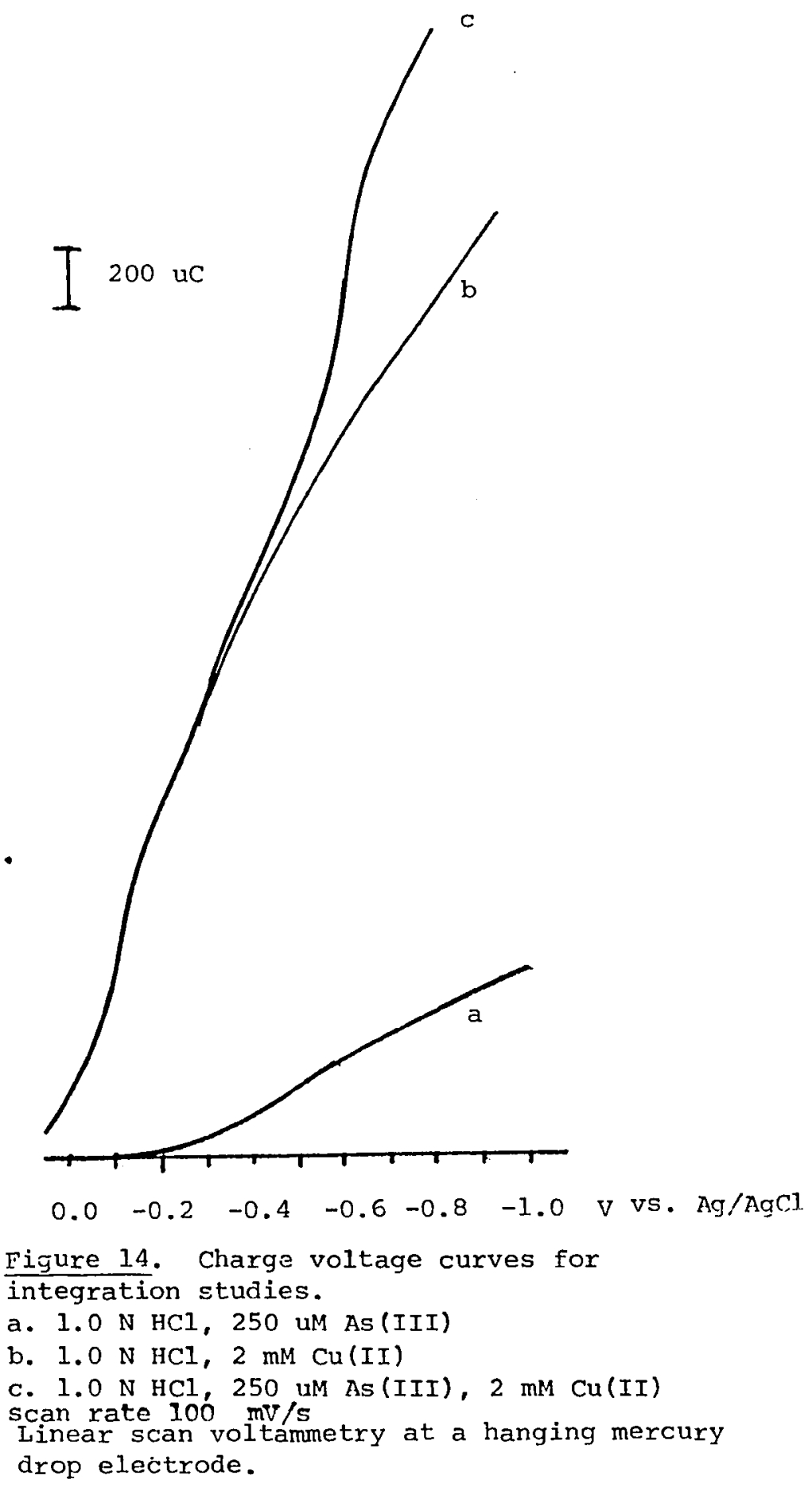


In the preliminary experiments it was observed that the addition of catechol to the analytical solution reduced the size of the arsenic cathodic stripping peak. Experiments were conducted to study the effect of catechol on the cathodic stripping with copper codeposition of arsenic.

A solution was prepared which contained $1.0 \mathrm{~N} \mathrm{HClO}$ and $0.1 \mathrm{mM}$, $6.35 \mathrm{ppm} \mathrm{Cu}(\mathrm{II})$. Linear voltammograms were recorded of the reduction of $\mathrm{Cu}(\mathrm{II})$ at a HMDE. The initial potential was $+0.25 \mathrm{~V}$ versus the $\mathrm{Ag} / \mathrm{AgCl}$ reference electrode and the scan was conducted at a rate of $-100 \mathrm{mV} / \mathrm{s}$. A recording of the $\mathrm{Cu}(\mathrm{II})$ reduction can be seen in Figure 15, curve a. The reduction peak potential for $\mathrm{Cu}(\mathrm{II})$ was found to be $-0.1 \mathrm{~V}$ versus the $\mathrm{Ag} / \mathrm{AgCl}$ reference electrode. Solid catechol was then added to the $\mathrm{Cu}(\mathrm{II})$ solution to produce a concentration of $0.5 \mathrm{M}$. Another linear scan was recorded and shown in Figure 15, curve b. It can be seen that the $\mathrm{Cu}(\mathrm{II})$ reduction peak shifts significantly in the cathodic direction and is found at a potential of $-0.67 \mathrm{~V}$ versus the $\mathrm{Ag} / \mathrm{AgCl}$ reference electrode. This cathodic shift in the peak potenial is quite significant and accounts for the loss of sensitivity of cathodic stripping of As(III) with copper codeposition. When deposition potentials of $-0.6 \mathrm{~V}$ are applied the $\mathrm{Cu}(\mathrm{II})$ does not reduce to form copper-amalgam in solutions of $0.5 \mathrm{M}$ catechol. Cathodic stripping of As(III) is not effective without the formation of copper-amalgam during the deposition step. In addition the $\mathrm{Cu}($ II) reduction peak current has decreased by $22 \%$.

To solve the problem of the cathodic shift in the $\mathrm{Cu(II)} \mathrm{reduction}$ potential a number of approaches were tested. These approaches can be 


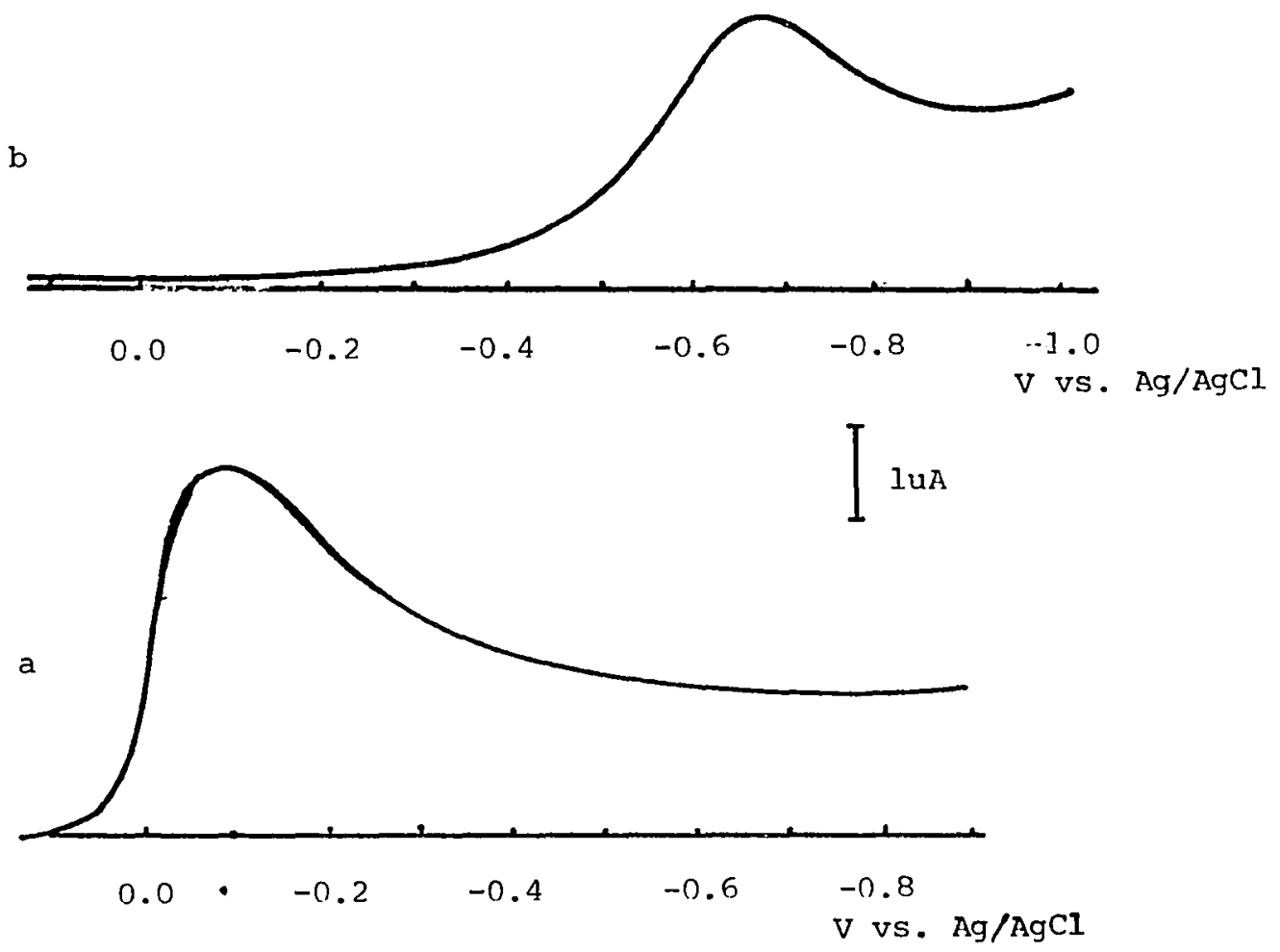

Figure 15. Effect of catechol on the reduction of Cu(II).

a. $1.0 \mathrm{~N} \mathrm{HClO}_{4}, 6.35 \mathrm{ppm} \mathrm{Cu(II)}$

b. $1.0 \mathrm{~N} \mathrm{HClO} 4,6.35 \mathrm{ppm} \mathrm{Cu}($ II), $0.5 \mathrm{M}$ catechol Linear scan voltammetry at hanging mercury drop electrode, scan rate $=100 \mathrm{mv} / \mathrm{s}$. 
described as two different types; solution phase studies and working electrode studies. The solution phase experiments involved studying supporting electrolytes and copper ion speciation in hopes of finding a combination of electrolyte and copper ion which would resist reaction with catechol. Working electrode studies involved examination of metalamalgam electrodes such as copper wire-mercury film electrodes and their use in cathodic stripping voltammetry. The solution phase experiments will be described below. The metal-amalgam electrode experiments will be described in a later section.

The mineral acid recommended for acidification of aqueous samples for As(III) analysis by both Henze et al. (43) and Sadana (44) was hydrochloric acid. Sadana (44) reported that sulfuric acid is an unsuitable medium for cathodic stripping analysis. Apparently the choice of mineral acid has a great influence on the success of cathodic stripping experiments. The experiments illustrated in Figure 15 were conducted in perchloric acid. In perchloric acid solution the $\mathrm{Cu}(\mathrm{II})$ ion reacts with added catechol to form a complex which is more difficult to reduce than free $\mathrm{Cu}(\mathrm{II})$. The copper ions must be more easily reduced than the As(III) ions for successful cathodic stripping. To ensure the required order of copper and arsenic reduction potentials the copper ion must be protected from complexation with catechol. Proper choice of mineral acid and copper oxidation state could prevent copper reaction with catechol.

Experiments conducted in this study demonstrated that in high concentrations of $1.0 \mathrm{M}$, the chloride ion may successfully compete with catechol for reaction with $\mathrm{Cu}(\mathrm{I})$. The cuprous ion is strongly complexed 
by chloride ion resulting in the $\mathrm{CuCl}^{-1}$ fon with a reduction peak potential of approximately $-0.1 \mathrm{~V}$ versus the $\mathrm{Ag} / \mathrm{AgCl}$ reference electrode.

Linear scan voltammetry conducted on solutions of $1.0 \mathrm{~N} \mathrm{HCl}, 63.5$ $\mathrm{ppm} \mathrm{Cu}(\mathrm{I})$ without and with catechol demonstrated that the $\mathrm{CuCl}$ species resists reaction with catechol. The potential of the reduction peak does not change after catechol addition. In Figure 16 the use of $\mathrm{Cu}(\mathrm{I})$ in the cathodic stripping of $A s(V)$ is illustrated.

Copper (I) and Arsenic (V)

Copper (I) has been reported as a useful reductant for As(V) in aqueous solutions. Simon et al. (53) have recommended the use of $\mathrm{Cu}(\mathrm{I})$ for conversion of $A s(V)$ into As(III) for subsequent analysis. Experiments were conducted to determine if $\mathrm{Cu}(\mathrm{I})$ reduces $\mathrm{As}(\mathrm{V})$, converting it into As(III) in typical cathodic stripping conditions.

Solutions of $1.0 \mathrm{~N} \mathrm{HCl}, 2 \mathrm{mM}(127 \mathrm{ppm}) \mathrm{Cu}(\mathrm{I})$, and $0.5 \mathrm{mM}$ (37.5 ppm) As(V) were prepared. Each solution was deaerated by nitrogen gas. After mixing of the components the solution composition was monitored by periodic linear potential scans. Changes in the copper (I) concentration would be evidenced by a growth of the copper reduction peak. Conversion of the $A s(V)$ into As(III) would be evidenced by formation of a cathodic stripping peak for the As(III). These changes would correspond to the following oxidation-reduction reaction.

$$
2 \mathrm{Cu}(\mathrm{I})+\mathrm{As}(\mathrm{V}) \quad 2 \mathrm{Cu}(\mathrm{II})+\mathrm{As}(\mathrm{III})
$$

The conversion of $A s(V)$ to $A s(I I I)$ under these experimental 


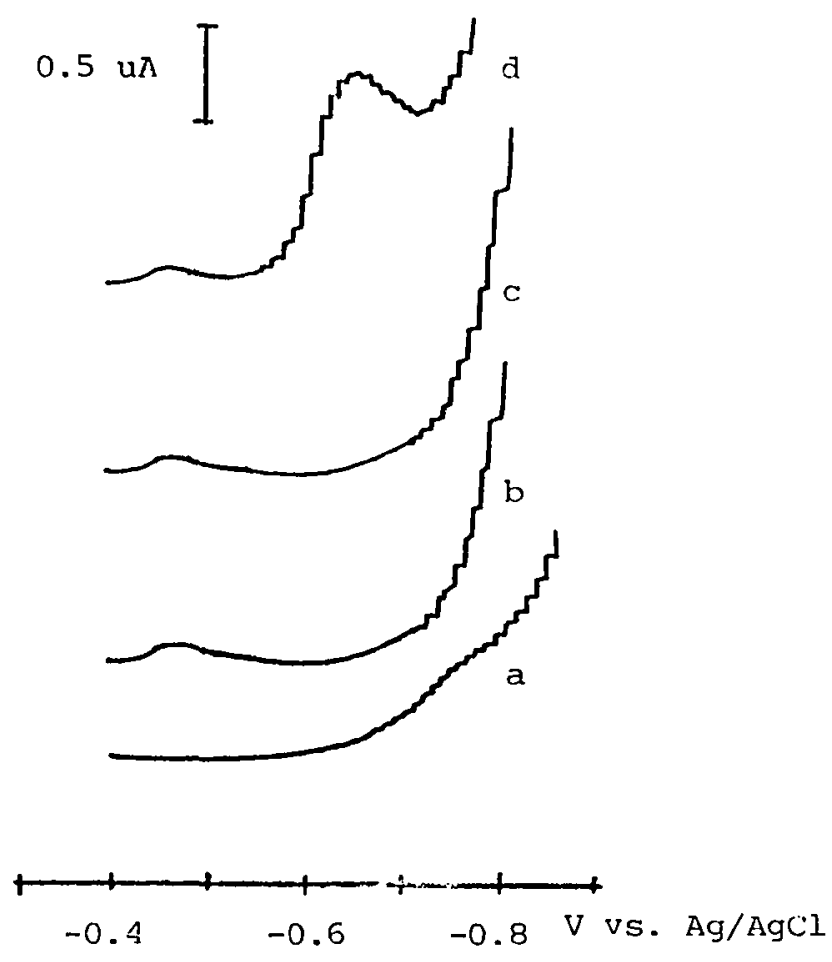

Figure 16. Differential pulse cathodic stripping voltammetry of $370 \mathrm{ppb}$ As (V) with $\mathrm{Cu}(\mathrm{I})$ codeposition in $0.5 \mathrm{M}$ catechol. a. $1.0 \mathrm{~N} \mathrm{HCl}$

b. $1.0 \mathrm{NHC1}, 6.35 \mathrm{ppm} \mathrm{Cu(I)}$

c. $1.0 \mathrm{~N} \mathrm{HCl}, 6.35 \mathrm{ppm} \mathrm{Cu}(\mathrm{I}), 0.5 \mathrm{M}$ catechol d. $1.0 \mathrm{~N} \mathrm{HCl}, 6.35 \mathrm{ppm} \mathrm{Cu}(\mathrm{I}), 0.5 \mathrm{M}$ catechol, $370 \mathrm{ppb}$ As (V) deposition potenetial $=-0.4 \mathrm{~V}$ deposition period $=1$ minute scan rate $=10 \mathrm{mV} / \mathrm{s}$ pulse height $=50 \mathrm{mV}$ 
conditions was not observed. Detailed results of these experiments shall be presented later.

Metal Amalgam Electrodes

Cathodic stripping should be possible at metal-amalgam electrodes composed of solid metal electrodes plated with a thin mercury film. At these working electrodes the metal-amalgam has already formed and no free metal ion should be neccessary for successful cathodic stripping of As(III). Experiments were conducted with copper, nickel and palladium electrodes coated with a thin film of mercury.

A copper electrode was prepared for evaluation. This electrode consisted of a $1 \mathrm{~mm}$ diameter copper wire embedded in a non-conducting epoxy. The epoxy electrode was 0.5 inch in diameter with the copper wire embedded in the center. The electrode surface was cut perpendicular to the length of copper wire and polished with an alumina slurry to produce a microscopically smooth surface. A thin mercury film was deposited from a $1.0 \mathrm{~N} \mathrm{HClO}$ solution containing $1.0 \mathrm{mM} \mathrm{Hg}$ (II). A deposition potential of $-0.4 \mathrm{~V}$ was applied for 2 minutes to produce a thin mercury film. The film was conditioned by cyclic scans in the potential range of $0.0 \mathrm{~V}$ to $-1.0 \mathrm{~V}$. The reproducibility of cathodic stripping experiments performed at the copper-amalgam electrode was tested using solutions of $1.0 \mathrm{~N} \mathrm{HClO}$ and $55 \mathrm{uM}, 4.2 \mathrm{ppm}$ As(III). The arsenic was deposited at a potential of $-0.5 \mathrm{~V}$ for 1 minute. The arsenic was then cathodically stripped by a linear scan. The current was integrated during arsenic deposition and stripping. The measured charge corresponds to the amount of arsenic deposited in addition to 
other faradic and nonfaradic contributions of trace components. An example of a stripping peak produced at a copper amalgam electrode can be seen in Figure 17 .

The use of copper-amalgam electrodes in catechol solutions was also examined. The copper-amalgam electrode was used as the working electrode in a solution of As(III): Cathodic stripping voltammetry was conducted on the As(III) solution. Solid catechol was then added to the As(III) solution to produce a concentration of 0.5 M. Cathodic stripping voltammetry was again performed. Cathodic stripping peaks were observed for As(III) at the copper-amalgam electrodes both in solutions with catechol and without. In solutions of catechol the cathodic stripping peak was significantly reduced in size.

In addition to study of the use of copper-amalgam electrodes other metals were considered as possible aids for cathodic stripping of As(III). Solid metal-amalgam electrodes composed of nickel or paladium were also tested. No evidence of an arsenic cathodic stripping peak was observed when nickel or paladium electrodes were used in As(III) solutions.

Cathodic Stripping with Codeposition of $\mathrm{Ni}, \mathrm{Pd}$, and $\mathrm{Fe}$

The effect of added $\mathrm{Ni}(\mathrm{II}), \mathrm{Pd}(\mathrm{II})$, and $\mathrm{Fe}(\mathrm{III})$ on the cathodic stripping of As(III) was examined. Cathodic stripping with codeposition of $\mathrm{Ni}(\mathrm{II}), \mathrm{Pd}(\mathrm{II})$, or $\mathrm{Fe}(\mathrm{III})$ was conducted on $2 \mathrm{mM}$ solutions of the added ion. Cathodic stripping peaks were observed for all of the metal ions examined. The arsenic stripping peak was relatively small and close to the hydrogen wave for solutions of Pd(II) 


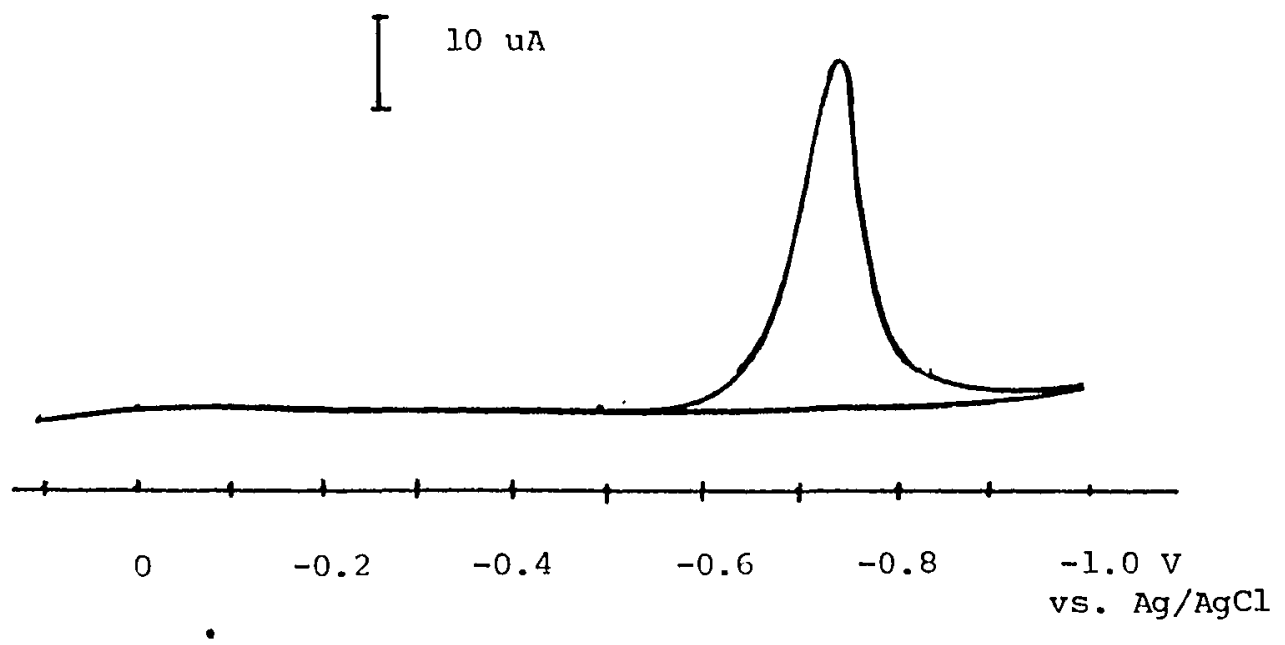

Figure 17. Cathodic stripping performed at a copperamalgam electrode. Deposition Potential $=-0.5 \mathrm{~V}, 4.2$ ppm As(III). $1.0 \mathrm{~N} \mathrm{HCl}$ electrolyte. Deposition period = $2 \mathrm{~min}$ stir/ $0.5 \mathrm{~min}$ quiet. Scan rate $=50 \mathrm{mv} / \mathrm{s}$. 
and Fe(III). Solutions of $\mathrm{Ni}$ (II) produced strong cathodic stripping signals but again the stripping peak was close to the hydrogen evolution wave. In solutions of low arsenic concentrations the hydrogen wave masks the stripping peak resulting in poor sensitivity for cathodic stripping voltammetry with nickel codeposition. 
Method for Speciation of Inorganic Arsenic

The electrochemical method chosen to measure low, environmental. levels of $A s(I I I)$ and $A s(V)$ must be very sensitive. The technique of cathodic stripping voltammetry with copper codeposition was chosen for application to the determination of $A s(V)$ concentrations.

A procedure was developed for measurement of inorganic arsenic in aqueous samples. Solution conditions were chosen to maximize the sensitivity of analysis procedures for both $\Lambda s$ (III) and As(V). Water samples were acidified with aliquots of concentrated, ultra pure $\mathrm{HCl}$ to produce a concentration of $1.0 \mathrm{~N}$ HCl. To determine the As(III) concentration aliquots of $\mathrm{Cu}(\mathrm{I})$ stock solution were added to the acidified sample. The resultant $\mathrm{Cu}_{\mathrm{U}}(\mathrm{I})$ concentration was equal to 8 ppm. Cathodic stripping voltammetry was then conducted as in the …

methods described by Sadana (44).

To analyze for $\Lambda s(V)$ in water samples catechol must be added to the water sample to produce the EAC. Appropriate electrochemical. techniques such as $\operatorname{CSV}$ can then be used to monitor the EAC and determine its concentration. The concentration of the electroactive complex can then be used to determine the $\Lambda s(V)$ concentration from a calibration curve prepared from standard solutions. Experiments were conducted to develop a method for determination of $A s($ III), and As(V) separately, and the combined As(III) and As(V) concentrations in water samples.

The procedure for preparation of the electroactive complex is a critical step in the analysis of As(V). The resultant solution's condition should favor formation of the EAC in the highest possible 
concentration. One problem is that the reaction conditions (high acid, high catechol) which promote high EAC concentrations also result in rapid decay of EAC to reach equilibrium. One of the three chemfals needed for formation of the EAC; (As(V), catechol, or hydrogen ion) must be added rapidly to the analysis mixture followed quickly by electrochemical analysis within 30 seconds. The arsenic is already present in the water samples to be analyzed and so is not added to the mixture during analysis. Either the catechol or hydrochloric acid could be added as the final reagent to form the electroactive complex. Experiments were conducted to test the feasability of adding catechol as the final reagent for EAC formation.

The analysis procedure for inorganic arsenic in water samples contains 2 steps. The first step involves analysis of the water sample for As(III) concentration. The analysis conditions require acidification of a $5.0 \mathrm{~mL}$ aliquot of the water sample. The resultant solution concentration was $1.0 \mathrm{~N} \mathrm{HCl}$. Then an aliquot of $\mathrm{Cu}(\mathrm{I})$ stock solution is added to produce a concentration of $8 \mathrm{ppm} \mathrm{Cu}(\mathrm{I})$. The arsenite ion is determined by CSV. A deposition potential of $-0.4 \mathrm{~V}$ versus the $\mathrm{Ag} / \mathrm{AgCl}$ reference electrode was applied for 1 minute (30 seconds strirred-30 seconds quiet). The arsenic is stripped by a differential pulse cathodic scan at the rate of $10 \mathrm{mV} / \mathrm{s}$. The peak height is measured and compared to a standard curve for quantification of the As(III) concentration.

In step II of the analysis procedure catechol is added to the above solution to permit determination of the $A s(V)$ concentration. This step requires rapid, (within 30 seconds), addition and mixing of the 
catechol with the analysis solution. To achieve rapid mixing the catechol was added as a mixture containing $1.0 \mathrm{~N} \mathrm{HCl}, 8 \mathrm{ppm}$ Cu(J) and 1.0 M catechol. The catechol mixture was prepared in bulk volumes of 50 $\mathrm{mL}$ to $100 \mathrm{~mL}$. The solution was deaerated by bubbling nitrogen gas into the mixture. For each As(V) analysis $5.0 \mathrm{~mL}$ aliquots of the catechol mixture was added to the $5.0 \mathrm{~mL}$ water sample. The catechol mixture was injected into the analysis solution with a $10.0 \mathrm{~mL}$ glass syringe fitted with a flexible plastic tip. The flexible plastic tip was easily inserted into the electrochemical cell for addition of catechol. The plastic tip also avoided contamination observed when a stainless steel syringe needle had been used in the past. Catechol is a strong "universal" complexing agent and it was observed that the catechol mixture was quite corrosive to steel parts. When this catechol mixture is added in equal volumes to the analysis solution the resultant concentrations will be $1.0 \mathrm{~N} \mathrm{HCl}, 8 \mathrm{ppm} \mathrm{Cu}(\mathrm{I})$, and $0.5 \mathrm{M}$ catechol.

Step II involves performing CSV on the new analysis solution prepared above. The solution conditions chosen are best suited for the formation of the EAC. The kinetic reaction of the EAC is timed from the addition of the catechol mixture. Analysis of the solution begins after a 30 second mixing period. The analysis technique employed is cathodic stripping voltammetry as described above for As(III) analysis. Cathodic stripping voltammetry was performed by applying a deposition potential of $-0.4 \mathrm{~V}$ for 1 minute followed by a differential pulse cathodic scan. The first analysis commenced 30 seconds after addition of catechol, when the deposition potential of $-0.4 \mathrm{~V}$ was applied. The first cathodic stripping scan was recorded at time equals 1 minute and 30 seconds, 
after the first deposition period was complete. Then additional cSV analyses are performed every few minutes until equilibrium is reached, (approximately 12 minutes). The peak height measured from the CSV recordings is then used to determine the As(V) concentration. The calculations used will be described in the results and discussion chapter.

Experiments were conducted on a series of known solutions. The analytical method outlined above was tested on solutions of As(III) alone, As(V) alone, variable As(III) concentrations with constant As(V) levels, and variable As(V) concentrations with constant As(III) levels. 
EXPERIMFNTAL RESULTS

The results of the experiments described in the previous chapter are presented in this section under the headings: Electroactive Complex Studies, Differential Pulse Polarographic Studies of As(III) and As(V) in the Presence of Catechol, and Stripping Voltammetry.

\section{ELECTROACTIVE COMPLEX STUDIES}

Experiments were conducted to provide information on the identity of the electroactive complex. The $A s(V)$-catechol electroactive complex was formed by mixing solutions of acid, arsenic (V) and catechol to produce a kinetically active system. The electroactive complex, (EAC), is a transient species whose concentration was monitored by electrochemical methods. The data collected consisted of time dependent current measurements. The reduction current of the EAC is proportional to its concentration at the time of measurement. The change of current with time follows exponential decay as can be seen in Figure 18.

Exponential decay suggests a pseudo first order reaction mechanism for the disappearance of the EAC. This mechanism can be simply expressed as the following equation where the $\mathrm{E} \Lambda \mathrm{C}$ is converted into a higher order, electroinactive complex, (M), of unknown stoichiometry.

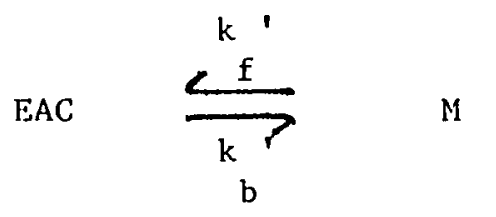

A rate expression for the above equation is written as follows. 


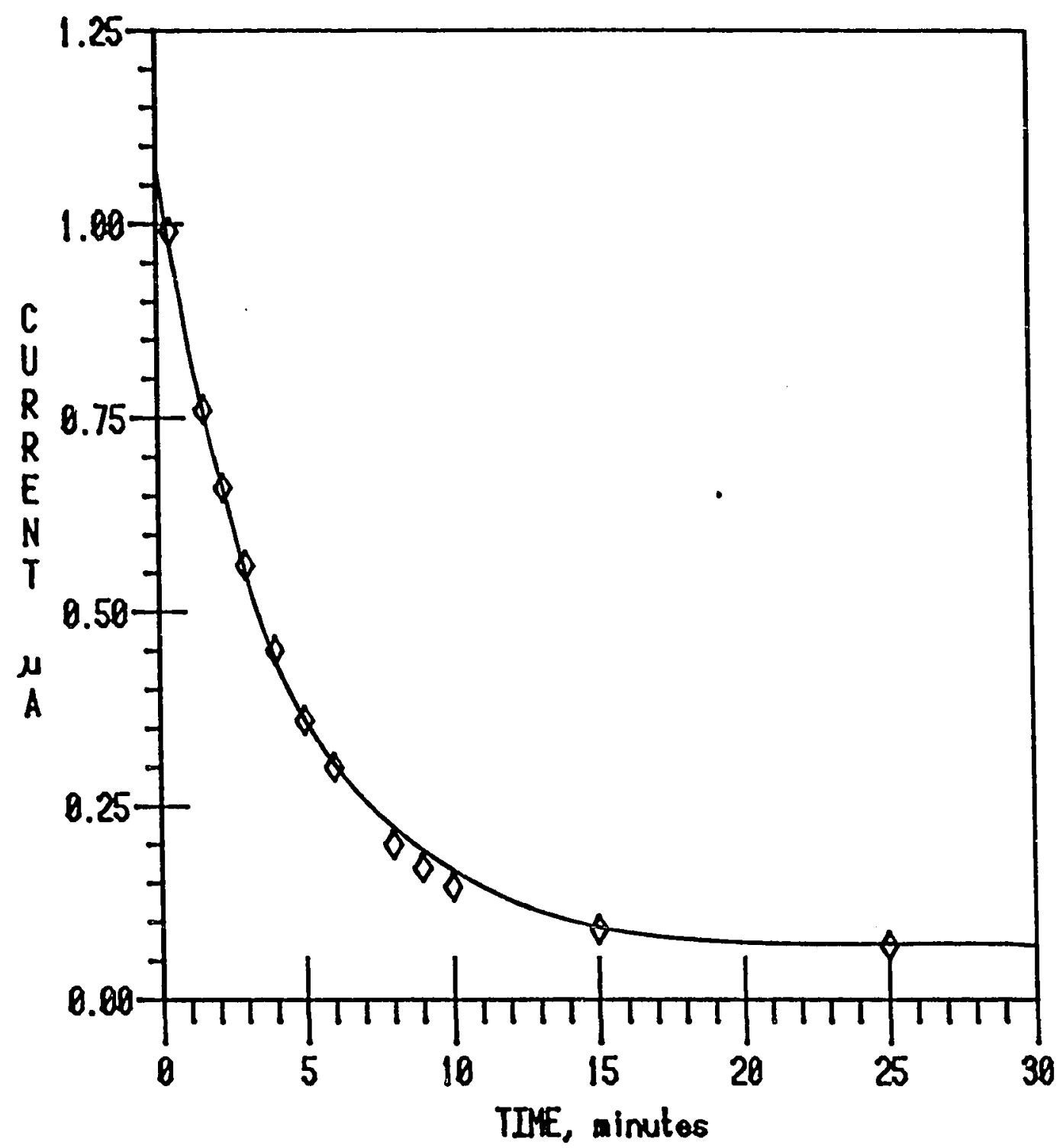

Figure 18 Current Decay of the EAC.

Reduction peak current as a function of time. $\mathrm{pH}=0$. Monitored by linear scan voltammetry at a hanging mercury drop electrode, scan rate $=50 \mathrm{mV} / \mathrm{s} .94 \mathrm{ppm} \mathrm{As}(\mathrm{V}), 0.5 \mathrm{M}$ catechol, 1.0 $\mathrm{N} \mathrm{NaClO}_{4}$, and $1.0 \mathrm{~N} \mathrm{HClO}_{4}$. 


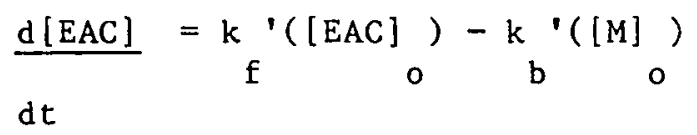

This equation can be integrated. Equation (7) shown below results when the assumptions that the concentration of the higher order complex, $M$, at the inftiation of the reaction, $t=0$, is equal to zero: [M] $=0$ at $t$ $=0$, and $[M]=[E A C]-[E A C]$ is used.

$$
\left(k^{\prime}+k_{b}^{\prime}\right) t=\ln \frac{[E A C]-[E A C]}{[E A C]-[E A C]}
$$

The reduction current, $i$, is proportional to the electroactive complex $\mathbf{t}$ at any time, $t$, and can be substituted into the above equation for the EAC concentration terms. The above equation can then be rearranged into a form suitable for graphical analysis.

$$
\ln \{i \underset{t}{-i}\}=-\left(k_{f}^{\prime}+k_{b}^{\prime}\right) t+\ln \{i-i\}
$$

The plot of $\ln \{i-i\}$ vs. time, $t$, has a slope of $-k$, the sum of the forward and reverse rate constants, $k=\left(k^{\prime}+k^{\prime}\right)$. The psuedo first order rate constant for the decay of the EAC is $k$ '. These calculations are performed after the current voltage curves recorded during the decay of the EAC have been measured. The reduction peak current is measured for each $\mathrm{CV}$ curve resulting in a specific value of i. The value of $i$ is calculated from the average reduction peak 
current measured when equilibrium is reached. It is assumed that equilibrium is reached when the reduction peak current no longer changes with time. This condition is typically achieved within 30 minutes of formation of the electroactive complex. The intercept of this graph is $\ln \{i-i\}$. This value can be used to calculate $i$, the current at time equals zero which is directly proportional to the As(V) concentration. In Figure 19 we can see an example of such a graph, calculated from data such as that presented in Figure 4. Since this plot is a straight line, it demonstrates that the disappearance of the EAC follows first order kinetics.

In solutions of higher $\mathrm{pH}$, such as $\mathrm{pH}=2$, the rate of formation of the electroactive complex has a finite value. The formation of the EAC is observed in Figure 20 as an increase in peak current immediately after the mixing of reaction components. The current rises to a maximum value and then decreases as the reaction progresses following an exponential decay. The rate of formation of the EAC depends on the hydrogen ion concentration as illustrated in Figure 20 , where a finite increase in peak current is observed prior to decay of the peak current at $\mathrm{pH}$ values of 2 . When the $\mathrm{pH}$ becomes lower, $\mathrm{pH}=1$, the rate of $\mathrm{EAC}$ formation is rapid and not observed, as seen in Figure 18, where the initial peak current is at a maximum value and exponential decay of the peak current is observed.

The values of the -intercept and slope of the kinetics plots vary as the catechol concentration and $\mathrm{pH}$ change. As described before the value of the intercept of the kinetics plot can be used to calculate the value of $i$, which is proportional to $A s(V)$ concentration. In Figure 21 


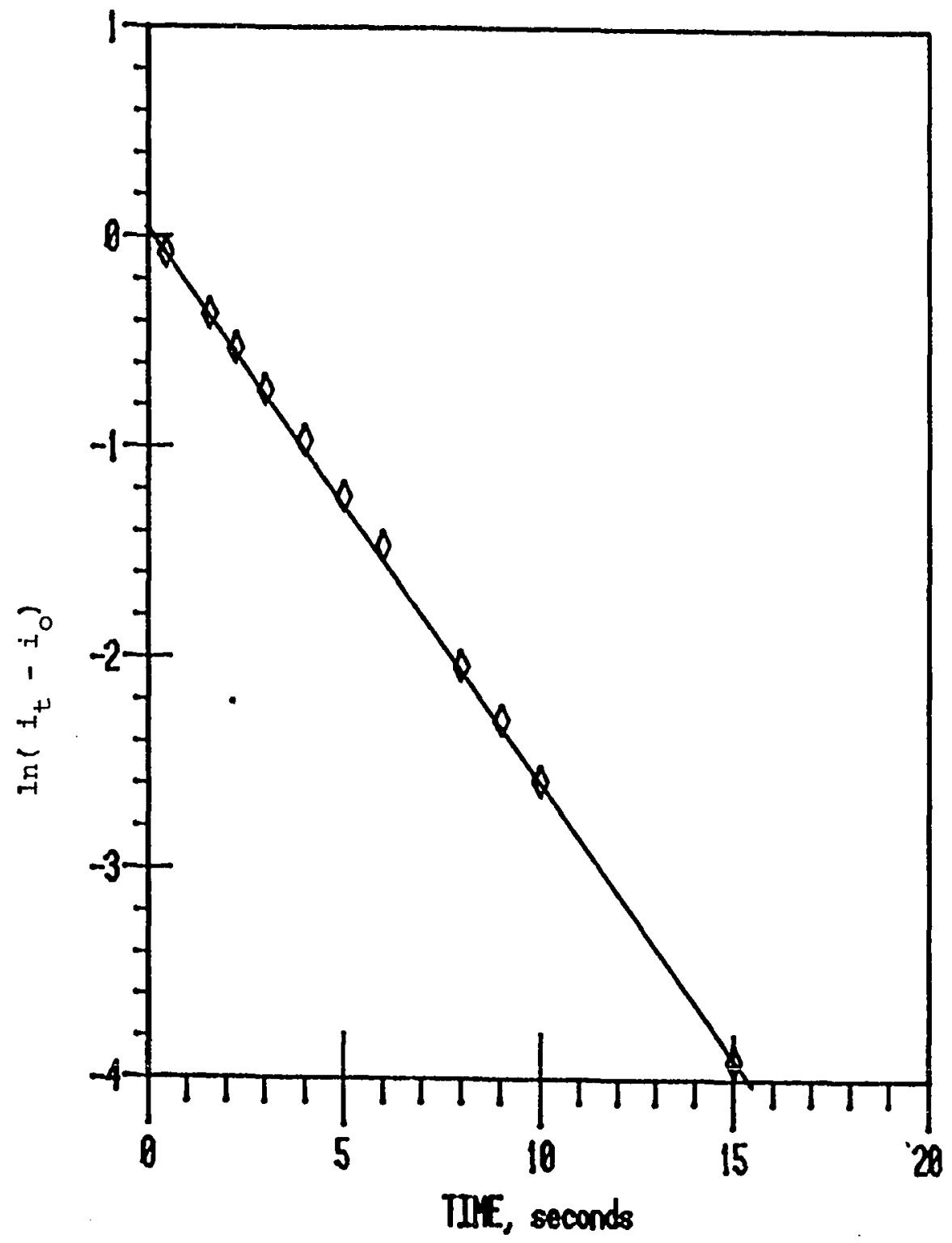

Figure 19. Kinetic plot of EAC decay current. Monitored by linear scan voltammetry at a hanging mercury drop electrode, scan rate $=50 \mathrm{mV} / \mathrm{s} .94 \mathrm{ppm}$ As (V) , $0.5 \mathrm{M}$ catechol, $1.0 \mathrm{~N} \mathrm{NaClO}_{4}$, and $1.0 \mathrm{~N} \mathrm{HClO}_{4}$. 


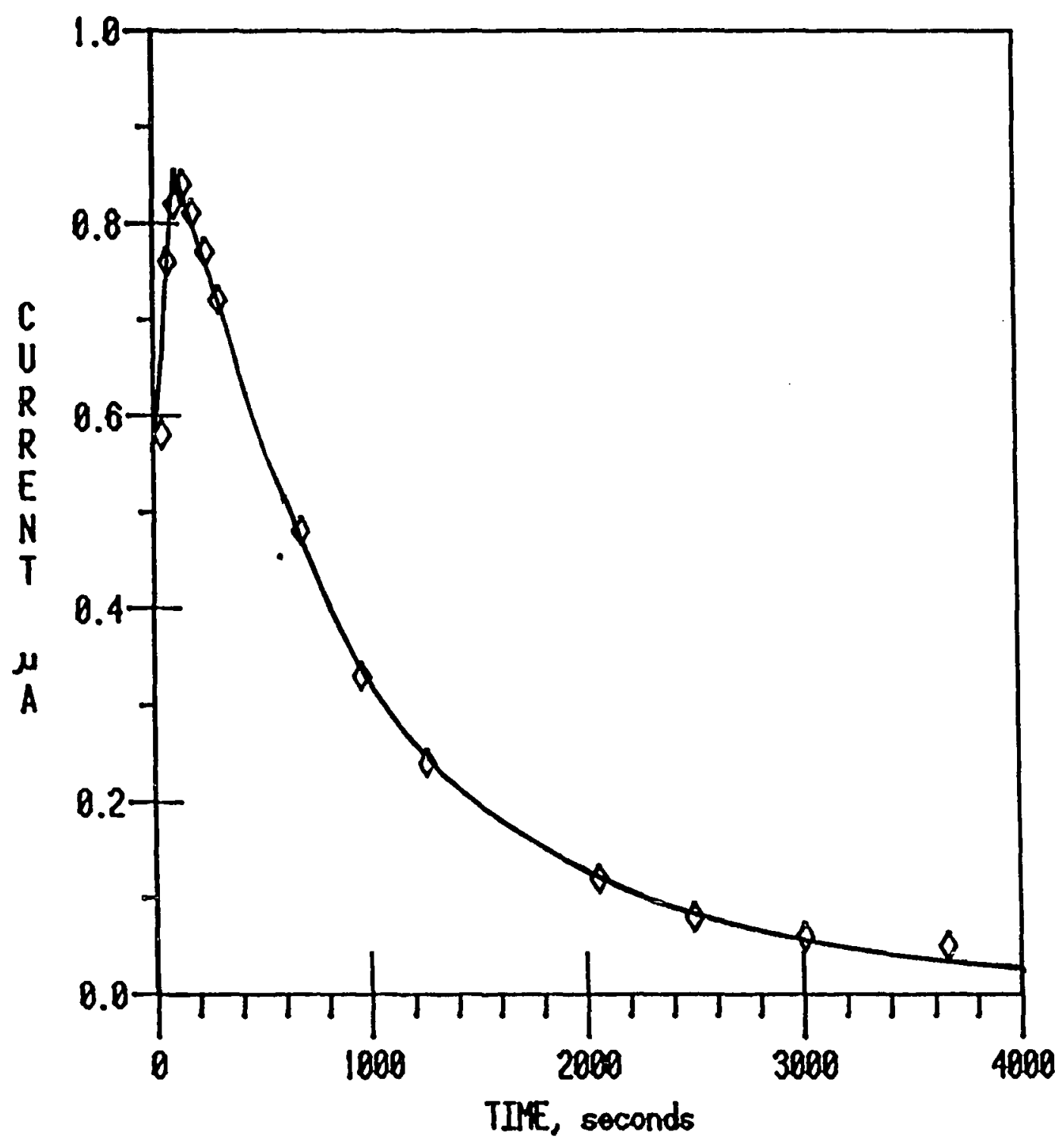

Figure 20. Current decay of the EAC.

Reduction peak current as a function of time. $\mathrm{PH}=2$.

75 ppm As (V); $0.5 \cdot \mathrm{M}$ catechol, and $1.0 \mathrm{~N} \mathrm{NaClO}_{4}$ Linear scan voltammetry at hanging mercury electrode, $\operatorname{scan}$ rate $=50 \mathrm{mv} / \mathrm{s}$. 
a plot of the value of $i$ is plotted as a function of catechol concentration for solutions of $1.25 \mathrm{mM} \mathrm{As}(V)$ is presented. The data represented in Figure 21 were calculated from experiments conducted in solutions of $0.1 \mathrm{~N} \mathrm{HCl}$. The data represented in Figure 22 was calculated from experiments conducted in solutions of $1.0 \mathrm{~N} \mathrm{HCl}$. The data presented here again demonstrates that the the hydrogen ion concentration has an affect on the value of $i$.

DIFFERENTIAL PULSE POLAROGRAPHIC STUdIES OF As(III) AND As(V) IN THE PRESENCE OF CATECHOL

Differential pulse polarography, (DPP), is a very sensitive technique for determination of $A s($ III) in a variety of environmental samples. This technique was used to measure the concentration of As(V) through complexation with catechol and formation of an electroactive complex. Preliminary experiments were conducted on solutions of As(III) and catechol to determine the influence of catechol on the DPP of As(III). Then DPP results were used to prepare a calibration curve for As(V) determination.

To evaluate the effect of catechol on As(III) a calibration curve was prepared for As(III) in a solution of $0.5 \mathrm{M}$ catechol and $1.0 \mathrm{~N} \mathrm{HCl}$. In Figure 8 a series of differential pulse polarographs of As(III)catechol solutions is presented. The data from these experiments were used to prepare a calibration curve for As(III) in $0.5 \mathrm{M}$ catechol. In Figures 23 and 24 the calibration curve is presented as a plot of reduction current versus As(III) concentration. The detection limit was determined by the method of Skogerboe et a1. (54). The calculated 


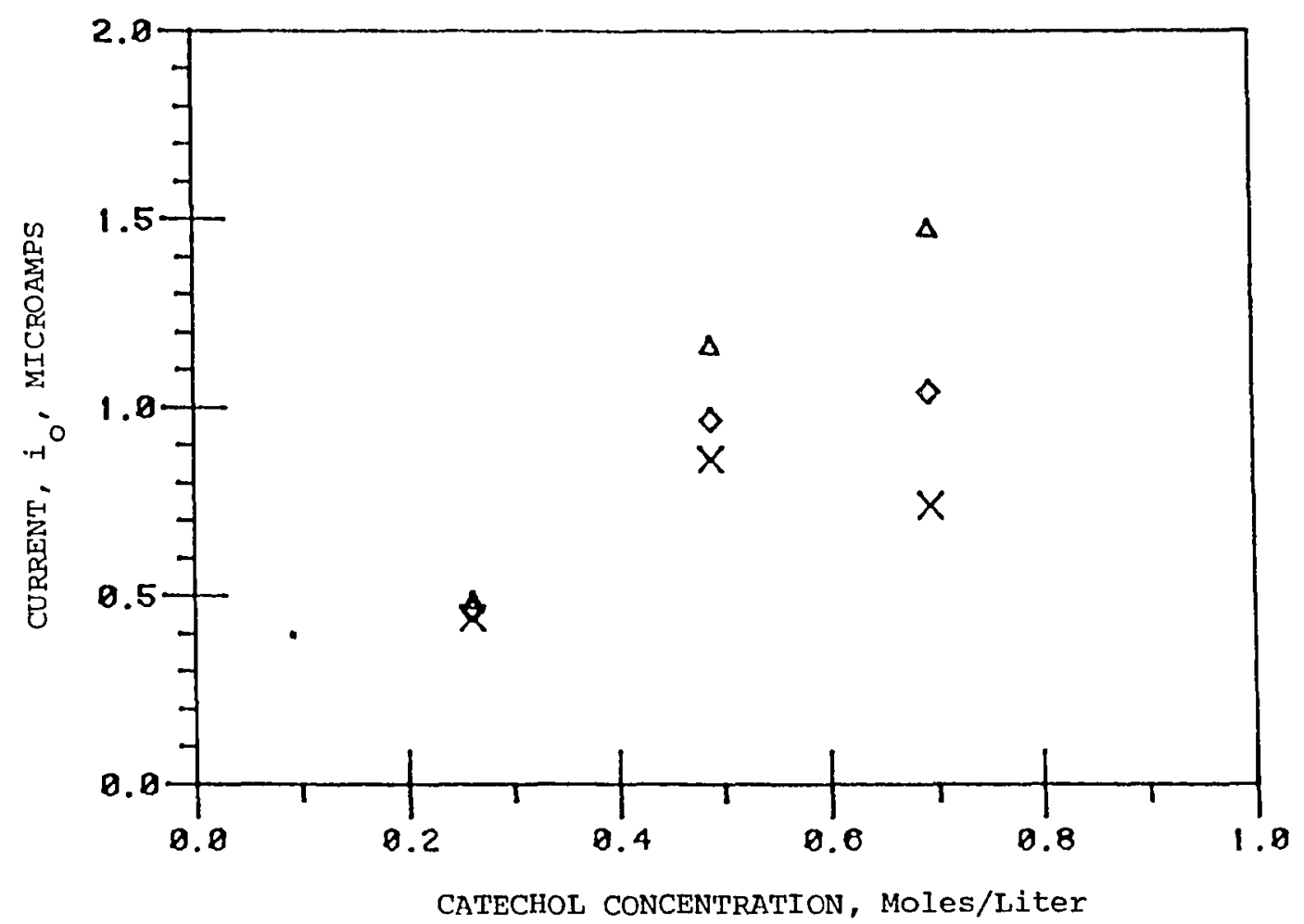

Figure 21. Initial current, $i$, as a function of catechol concentration in $1.0 \mathrm{~N} \mathrm{HCl}$ solution with $94 \mathrm{ppm} \mathrm{As}(\mathrm{V})$.

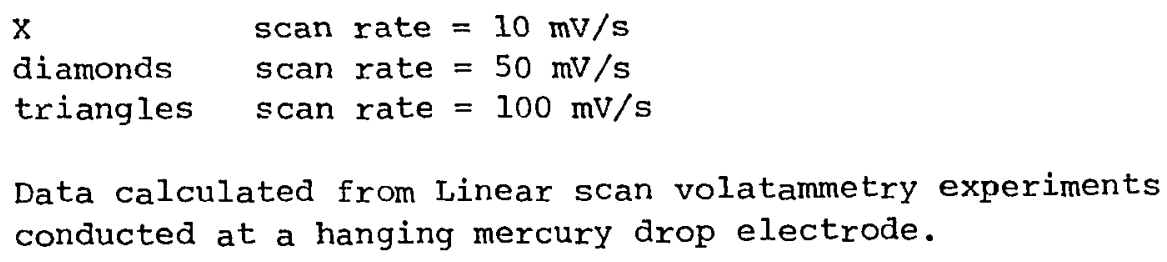




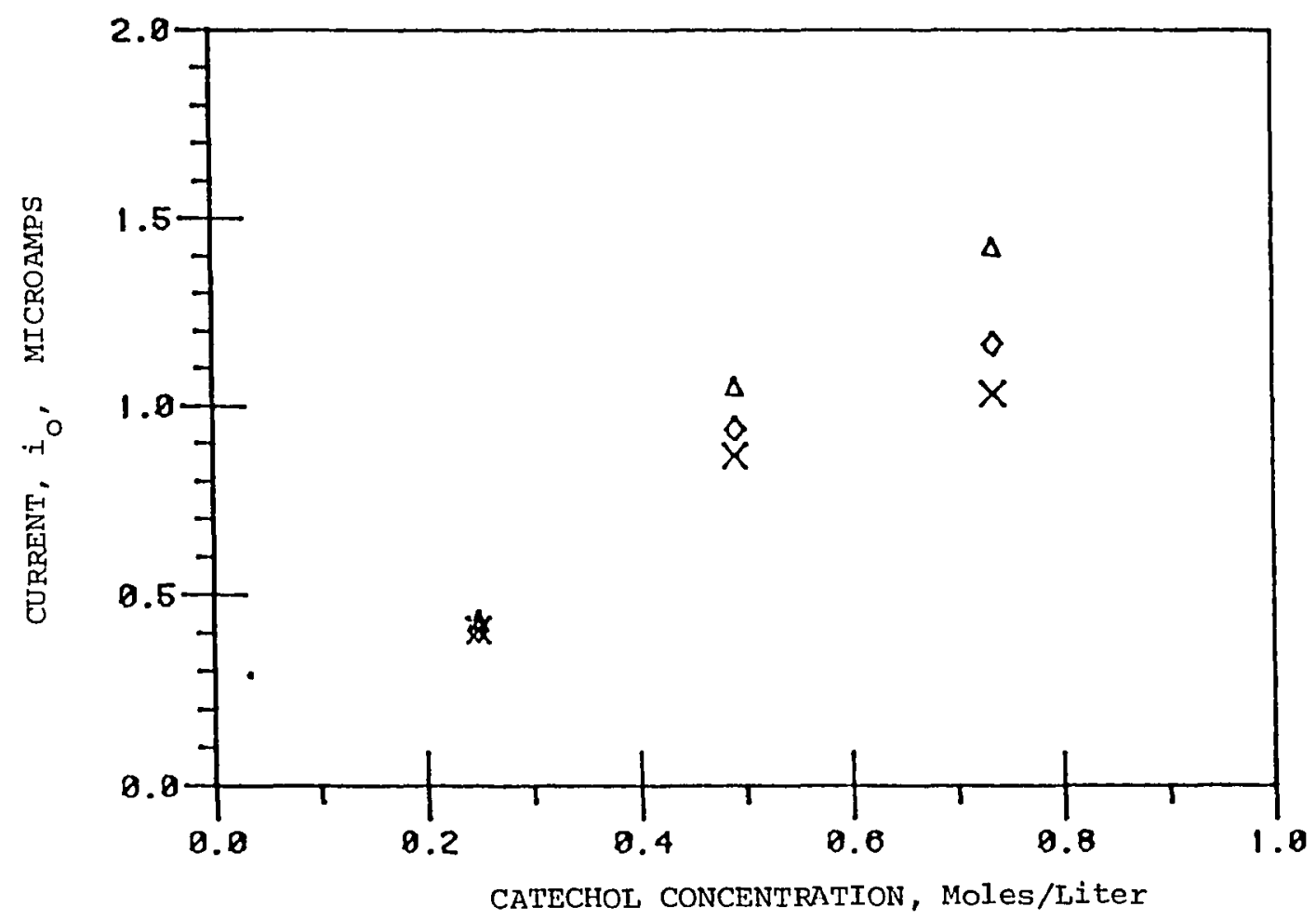

Figure 22. Initial current, $i_{o}$ as a function of catechol concentration in $1.0 \mathrm{~N} \mathrm{HCl}$ solution with $94 \mathrm{ppm} \mathrm{As}(\mathrm{V})$.

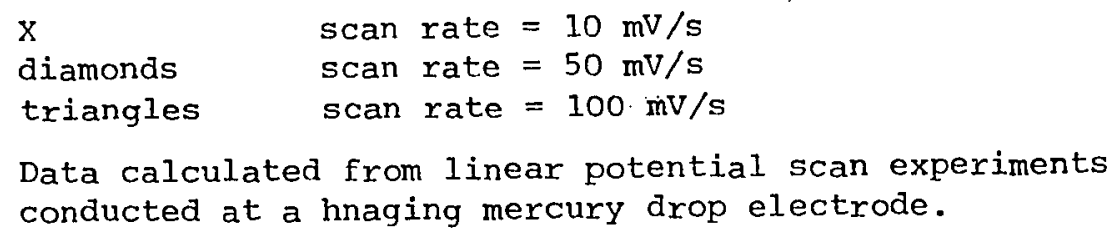




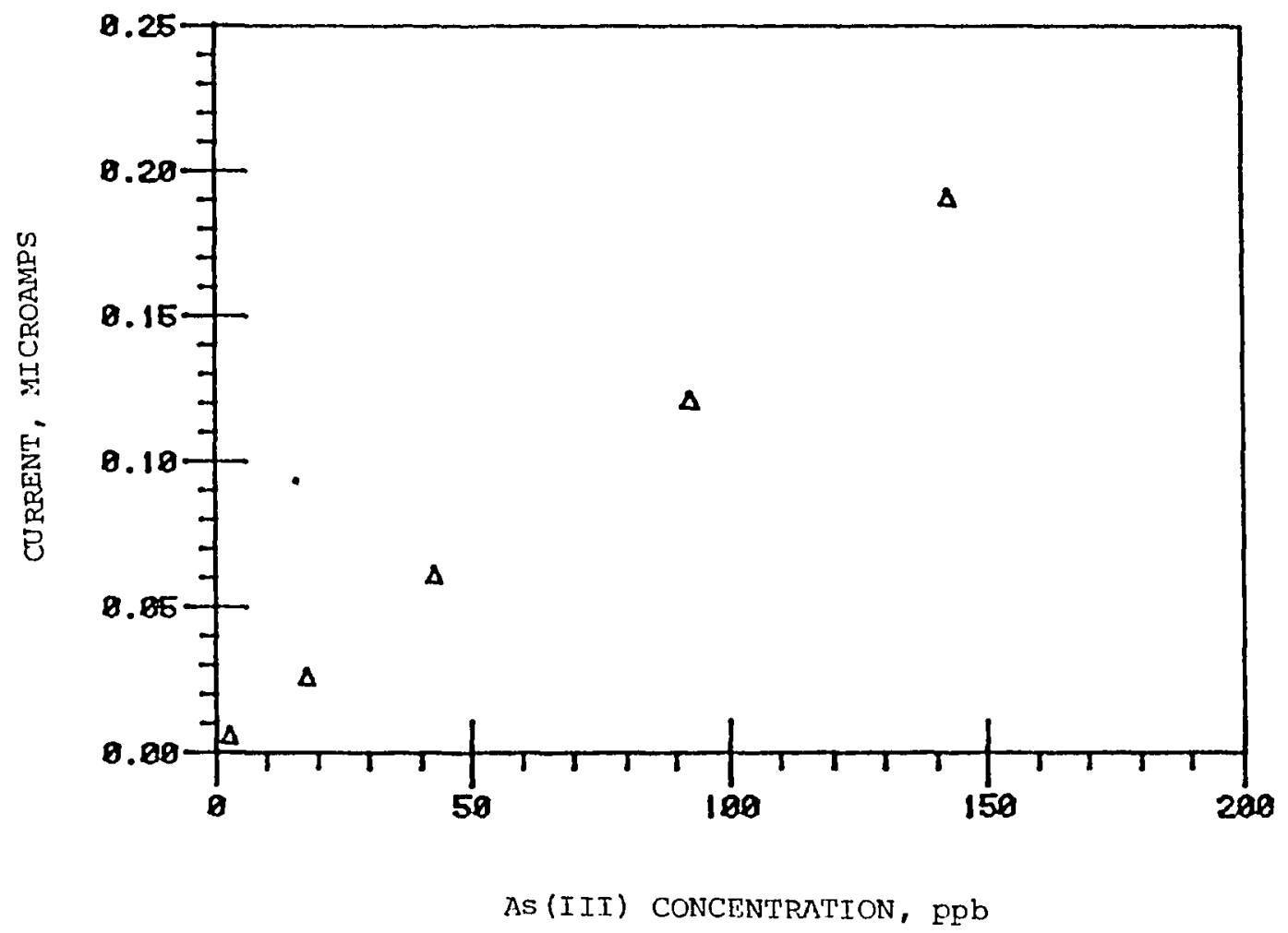

Figure 23. Calibration curve for analysis of $\lambda$ (III) in $0.5 \mathrm{M}$ catechol solution by differential pulse polarography. $0 \mathrm{ppb}$ to $200 \mathrm{ppb}$.

Dropping mercury electrode, pulse height $=100 \mathrm{mV}$, scan rate $=10 \mathrm{mV} / \mathrm{s}$. Supporting electrolyte $=1.0 \mathrm{~N} \mathrm{HCl}$. 


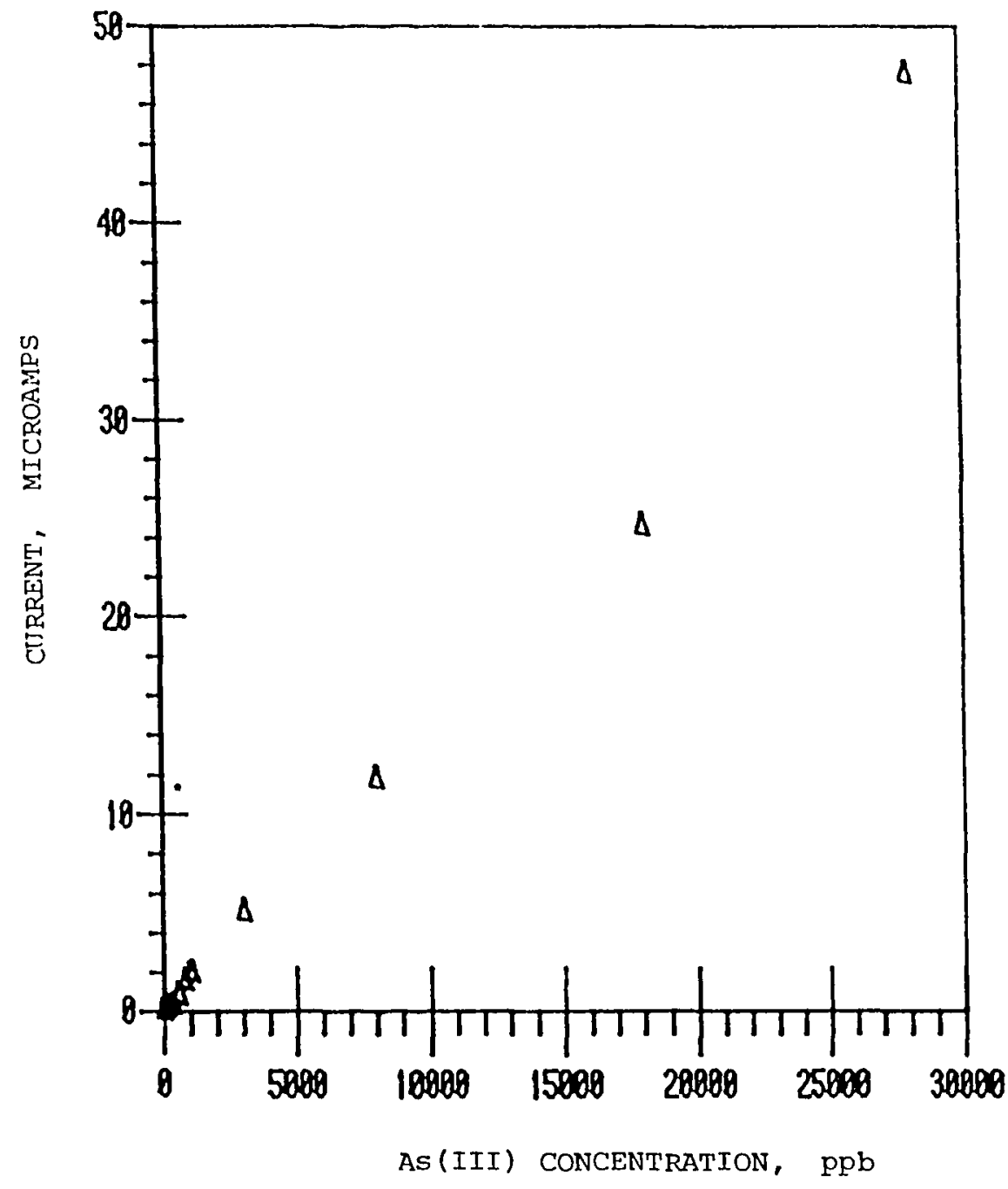

Figure 24. Calibration curve for analysis of As(III) in $0.5 \mathrm{M}$ catechol solution by differential pulse polarography.

Dropping mercury electrode, pulse height $=100 \mathrm{mV}$, scan rate $=10 \mathrm{mV} / \mathrm{s}$. Supporting electrolyte $=1.0$ $\mathrm{N} \mathrm{HCl}$. 
detection limit was found to be $3.1 \mathrm{ppb}$ As(III) at the $95 \%$ confidence level.

A calibration curve was also prepared for solutions of various As $(V)$ concentrations and $0.5 \mathrm{M}$ catechol. Each As(V) concentration was studied in an individual experiment. The EAC was monitored by application of a constant potential of $-0.40 \mathrm{~V}$. The current produced by reduction of the EAC was recorded on a strip chart recorder producing a record of reduction current versus time as seen in Figure 11. The strip chart recordings were then used in kinetic calculations to determine the value of $i$, which is proportional to the As(V) concentration. In Figure 25 the calibration curve for $A s(V)$ is presented as a plot of current, $i$ versus As(V) concentration. The detection limit was calculated by the same method. At the $95 \%$ confidence level the detection limit was calculated to be $0.24 \mathrm{ppm}$.

\section{STRIPPING VOLTAMMETRY}

The detection limits calculated for determination of As(V) by polarographic techniques are in the low $\mathrm{ppm}$ range. These high detection limits prevent the use of polarographic methods in determination of As(V) concentrations in environmental samples where arsenic levels are usually in the 1 to $100 \mathrm{ppb}$ range. Voltammetric stripping techniques were examined for application to the determination of As(V) concentrations at environmental levels.

Cathodic Stripping with Cu Codeposition

Two techniques have been reported for cathodic stripping 


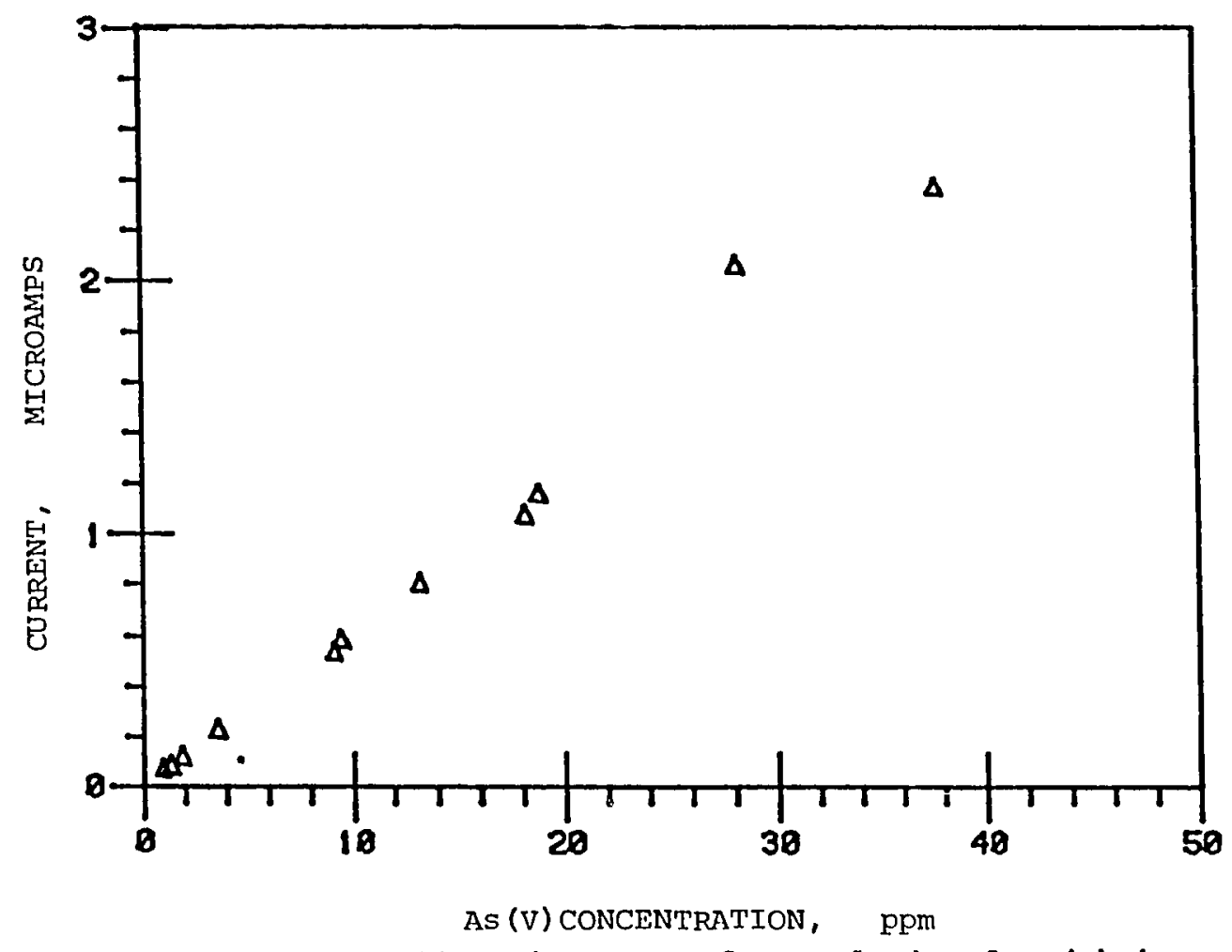

Figure 25. Calibration curve for analysis of As (V) in $0.5 \mathrm{M}$ catechol solution, determined by constant potential voltammetry. $E_{\text {applied }}=-0.4 \mathrm{~V}$ versus $\mathrm{\Lambda g} / \mathrm{AgCl}$ reference. Working electrode = dropping mexcury electrode supporting electrolyte $=1.0 \mathrm{~N} \mathrm{NaClO}_{4} / 0.1 \mathrm{~N} \mathrm{HClO}_{4}$ 
voltanmetric analysis of As(III) with copper codeposition $(43,44)$. Both methods require added $\mathrm{Cu}(\mathrm{II})$ and report very good sensitivities. Preliminary experiments were conducted to test the use of copper-aided cathodic stripping voltammetry for analysis of As(III) in solutions of catechol. In Figure 10 the cathodic stripping voltammograms for solutions of As(III), As(III) and $\mathrm{Cu}(I I)$, and $A s(I I I), C u(I I)$, and catechol are illustrated. The stripping peak current decreases by $33 \%$ in the presence of catechol.

The dependence of stripping peak height on copper concentration was examined. A solution of $0.14 \mathrm{uM}, 10.5 \mathrm{ppb}$ As(III) was treated with various concentrations of $\mathrm{Cu}(\mathrm{II})$. Cathodic stripping was conducted on each solution of different copper concentration. In Figure 26 the stripping peak current is plotted versus copper concentration. It can be seen that the peak current increases as the copper concentration increases, and furthermore, the large area of the peaks suggests that some other electrochemical reaction is also occuring.

Current integration experiments were conducted to determine if the stripping peak is indeed enhanced by another cathodic process. The experimental solutions were composed of $1.0 \mathrm{~N} \mathrm{HCl,} \mathrm{As}$ (III) and $\mathrm{Cu}($ II). Concentrations of the arsenic and copper were deliberately chosen so that the "stripping" peak was observed during linear potential scans without the need for a deposition step. In Figure 13 a sample of the typical current voltage" curves obtained in these experiments can be seen. The curves of charge versus applied potential presented in Figure 14 essentially represent the integration of the current versus potential curves in Figure 13. The charge for various solutions of As(III) and 


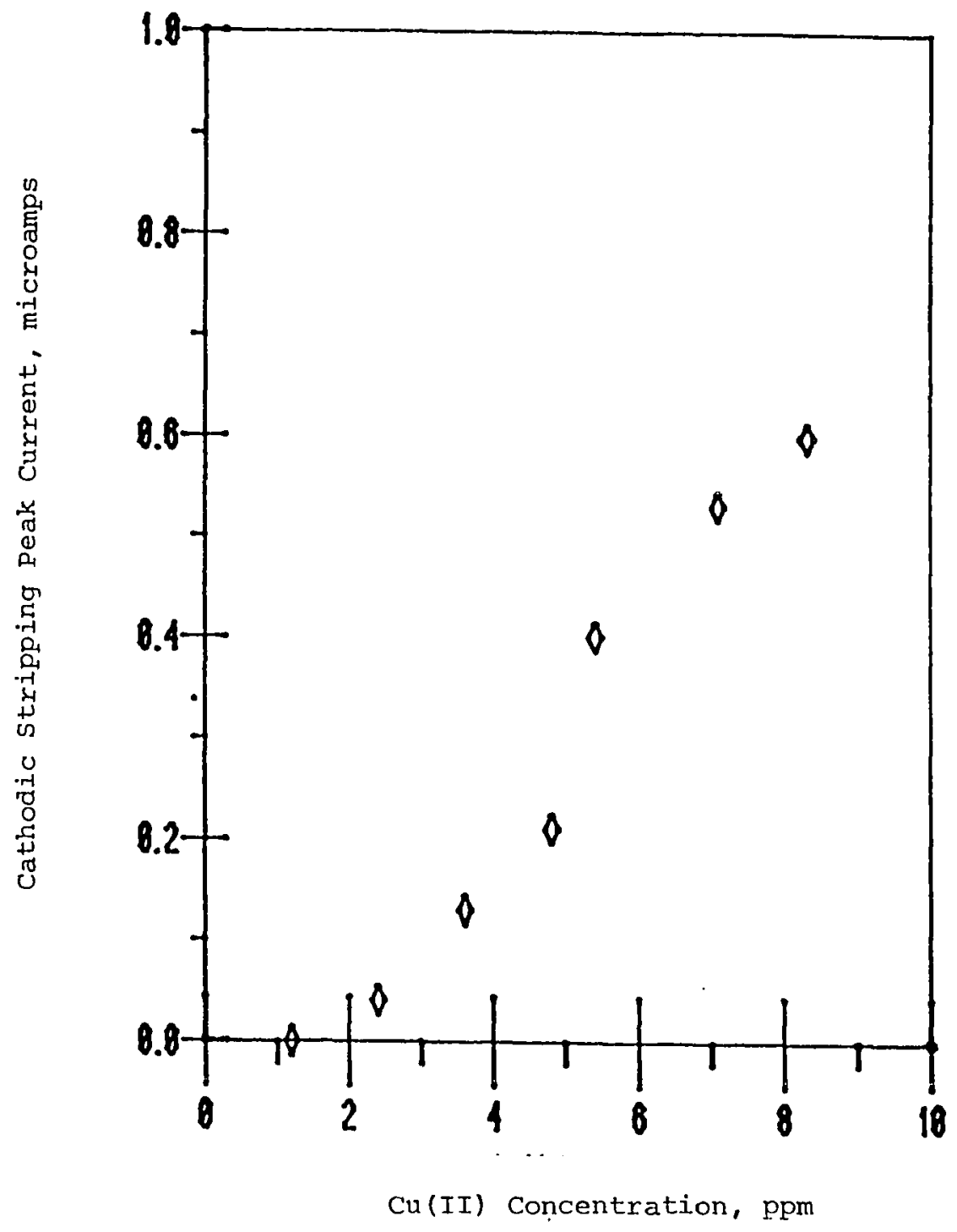

Figure 26. Dependence of cathodic stripping current on $\mathrm{Cu}$ (II) concentration. Solution conditions; $0.14 \mathrm{uM}, 10.5 \mathrm{ppb}$ As(III), $0.1 \mathrm{~N} \mathrm{HCl}$. Deposition period: 2 minutes

Deposition potential: $-0.5 \mathrm{~V}$ vs. $\mathrm{Ag} / \mathrm{AgCl}$ reference electrode.

Differential pulse cathodic strippina analvsis scan rate $=10 \mathrm{mV} / \mathrm{s}$, pulse height $=50 \mathrm{mV}$ 
$\mathrm{Cu}(\mathrm{II})$ was measured from the integrated curve and used to calculate stripping enhancement.

Each cathodic stripping integrated curve recorded can be described as the sum of the current integration for each component of the analysis solution. The analysis solution was composed of the components of supporting electrolyte, (E), arsentc(III), (As), and copper(II), (Cu). The charge from each component part was determined from evaluation of linear potential sweeps and current integrations recorded for each of the independent solutions of electrolyte, (E), arsenic and electrolyte, $(A s+E)$, and copper and electrolyte, $(\mathrm{Cu}+\mathrm{E})$. The experimental sum of the charge for a typical cathodic stripping solution was determined by recording current and charge curves for a solution composed of electrolyte, arsenic and copper, (As+Cu+E). The experimental value measured for a solution of arsenic, copper and electrolyte can be compared to the arithmetic sum of the component parts, this value shall be referred to as the (SUM). The difference between the experimental value of (As+Cu+E) and (SUM) represents the value of the stripping enhancement, and shall be referred to as (DIF). The equations below 11lustrate these calculations.

$$
\begin{aligned}
& (S U M)=(A s+E)+(C u+E)-(E) \\
& (D I F)=(A s+C u+E)-(S U M)
\end{aligned}
$$

The current enhancement observed for cathodic stripping with copper codeposition is graphically illustrated in Figure 27. The normalized excess charge, [DIF]/[As], is plotted versus the normalized copper concentration, [Cu]/[As]. When the ratio of copper to arsenic 


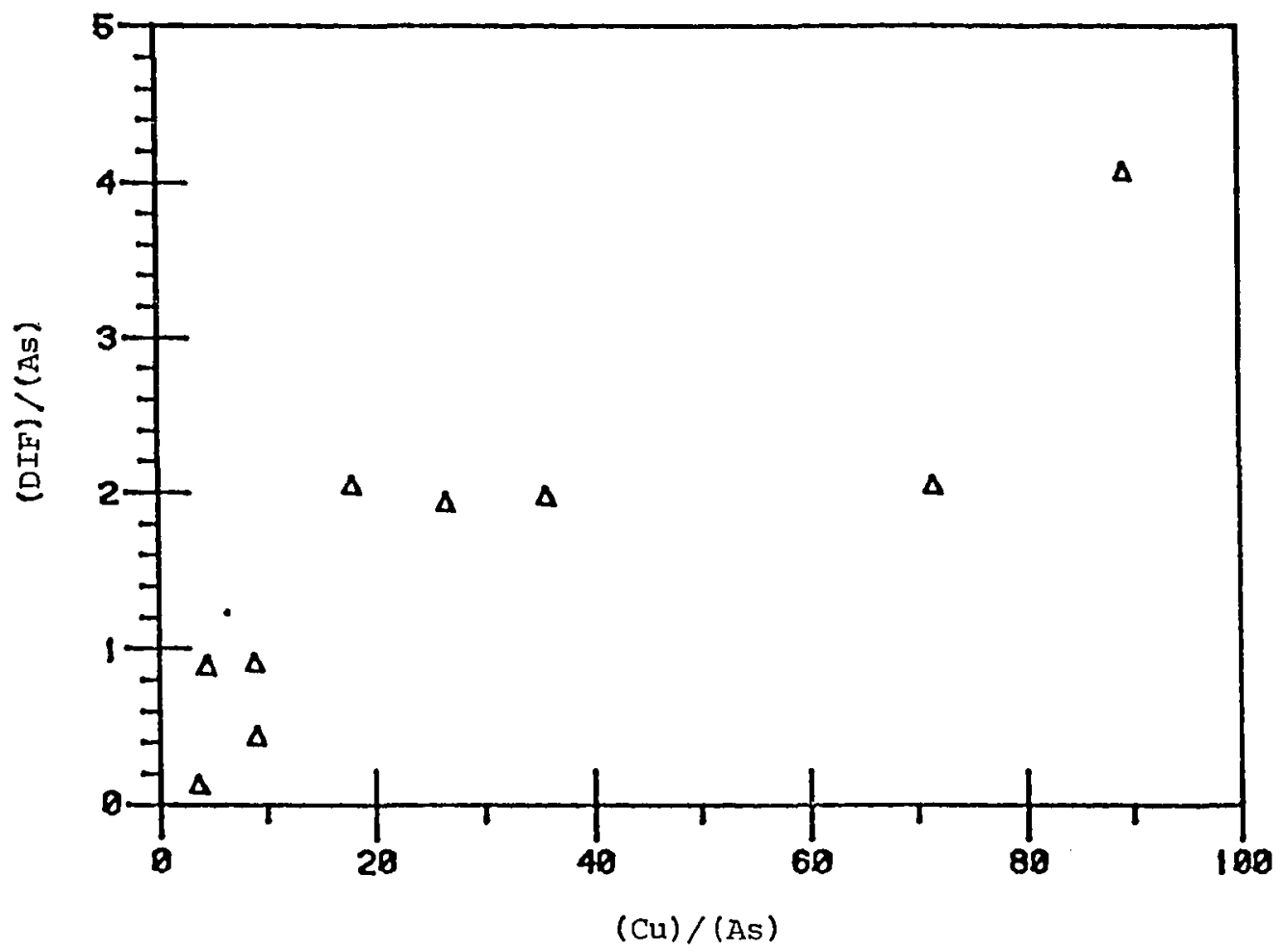

Figure 27. Dependence of normalized stripping enhancement, (DIF)/(As), on normalized copper concentration, (Cu) /(As).

Calculated from the data collected from cathodic stripping analysis of $\mathrm{As}$ (III) and $\mathrm{Cu}$ (II) solutions. Cathodic stripping analysis was performed at a hanging mercury electrode. 
is between zero and ten there is little enhancement observed. When the ratio of copper to arsenic increases to the range of 10 to 75 the current enhancement observed is essentially the constant value of 2 . An enhancement value of 2 means that the stripping current observed is $200 \%$ greater than the current expected for arsenic alone. When the normalized copper concentration increases above approximately 80 times greater than the arsenic level the enhancement begins to increases again. The region of uniform enhancement most probably corresponds to a concentration region where a specific arsenic-copper intermetallic is stable. The significant enhancement observed in these experiment is the source of the great sensitivity of these cathodic stripping techniques.

In order to apply the cathodic stripping methods developed for As(III) analysis to As(V) analysis the effect of catechol on these techniques must be examined. The preliminary results from cathodic stripping experiments conducted on solutions of As(III) and catechol indicate that the cathodic stripping peak current is reduced when catechol is added to analysis solutions of $\mathrm{Cu}($ II) and As(III). Experiments were conducted to identify the cause of the decrease in the stripping peak current.

In Figure 16 the recordings of linear potential scan experiments of $\mathrm{Cu}(\mathrm{II})$ solutions with and without catechol illustrate the dramatic impact of catechol on the reduction potential of $\mathrm{Cu}(\mathrm{II})$. Without catechol the $\mathrm{Cu}(\mathrm{II})$ reduces at approximately $+0.04 \mathrm{~V}$ versus the $\mathrm{Ag} / \mathrm{AgCl}$ reference electrode. When catechol is added to the solution the reduction potential shifts to $-0.67 \mathrm{~V}$ versus the $\mathrm{Ag} / \mathrm{AgCl}$ reference 
electrode. Cathodic stripping can be performed on As(III)-catechol solutions with the expected sensitivity in the absence of catechol if copper ion can be protected from reaction with catechol. Copper can be protected from reaction with catechol if it is in the form of $\mathrm{Cu}(I)$ and in the presence of high chloride ion concentration so that reaction with chloride ion occurs and the stable $\mathrm{CuCl}$ species is formed. The reduction potential of $\mathrm{Cu}(\mathrm{I})$ in $1.0 \mathrm{~N} \mathrm{HCl}$ remains at $+0.04 \mathrm{~V}$ versus the $\mathrm{Ag} / \mathrm{AgCl}$ reference electrode when catechol is added to the analysis solution. Figure 16 illustrates the use of $\mathrm{Cu}(\mathrm{I})$ for cathodic stripping of $A s(V)$ in catechol solutions. Copper ( $I$ ) in $1.0 \mathrm{~N} \mathrm{HCl}$ can be used for cathodic stripping of arsenic when catechol is necessary for As( $V$ ) analysis. 
In order to determine the concentration of As(V) by cathodic stripping with copper codeposition a number of special requirements must be met. The use of the EAC for detection of $A s(V)$ requires the presence of catechol in the analysis solution. As was observed above, the source of copper for cathodic stripping experiments conducted with catechol must be $\mathrm{Cu}(\mathrm{I})$. However, copper (I) has been recommended as a reducing agent for the conversion of As(V) into As(III) (53). Experiments were conducted to determine if $\mathrm{Cu}(\mathrm{I})$ is an effective reducing agent for $\mathrm{As}(\mathrm{V})$ under typical cathodic stripping conditions. Solutions of $1.0 \mathrm{~N} \mathrm{HCl}, 2$ $\mathrm{mM} \mathrm{Cu}(\mathrm{I})$, and $0.5 \mathrm{mM} \mathrm{As}(\mathrm{V})$ were monitored by periodic linear potential scans. The change in the copper reduction current over a period of 12 hours is reported in Table II.

TABLE II

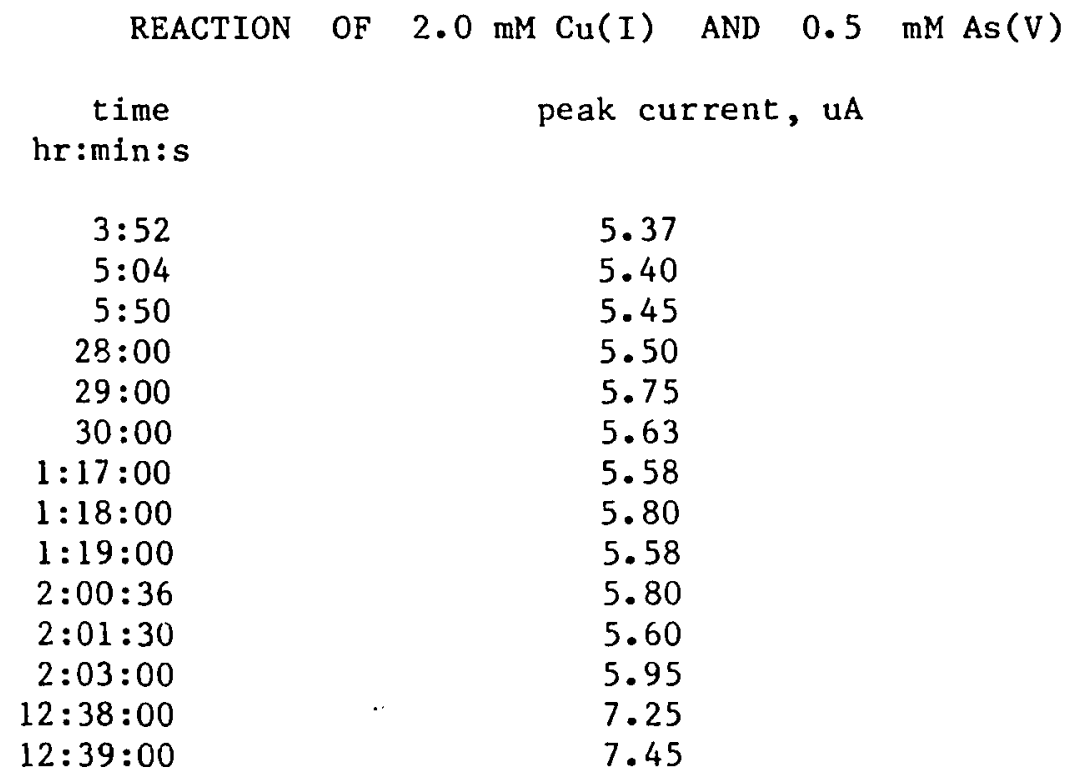

There was no evidence of a cathodic stripping peak in the experiment. After 12 hours the solution was treated with As(III) to 
produce a concentration of $0.56 \mathrm{mM}$ As(III). A dramatic stripping peak was then observed when As(III) was present in the solution in equal concentrations to the As(V).

Copper-electrodes were also examined for application to the cathodic stripping analysis of arsentc. The results of repetitive cathodic stripping experiments conducted on a 4.2 ppm solution of As(III) yielded an average stripping charge of 46.5 uC with a relative standard deviation of $18.8 \%$.

As(III) solutions with and without catechol were analyzed with copper-amalgam electrodes. The solution of As(III) without catechol had a stripping current of $1.6 \mathrm{~mA}$. When catechol was added to the As(III) solution the stripping peak was $0.89 \mathrm{~mA}$. The addition of solid catechol diluted the As(III) concentration by approximately $5 \%$ yet the stripping peak current was reduced by $45 \%$. The decrease in the stripping peak current may result from differences in the diffusion coefficients for As(III) and As(III)-catechol complexes present in catechol solutions.

Method for Speciation of Inorganic Arsenic

The procedure for analysis of As(III) and As(V) in aqueous solution involves two major steps. The analysis of As(III) is conducted in the first step; the sample is acidified, copper (I) is added, and cathodic stripping is performed. The As(III) concentration is then determined by comparing the stripping peak current to the appropriate calibration curve, Figure 28. In the second step a catechol solution is added to the analysis solution. The $A s(V)$ reacts with the added 


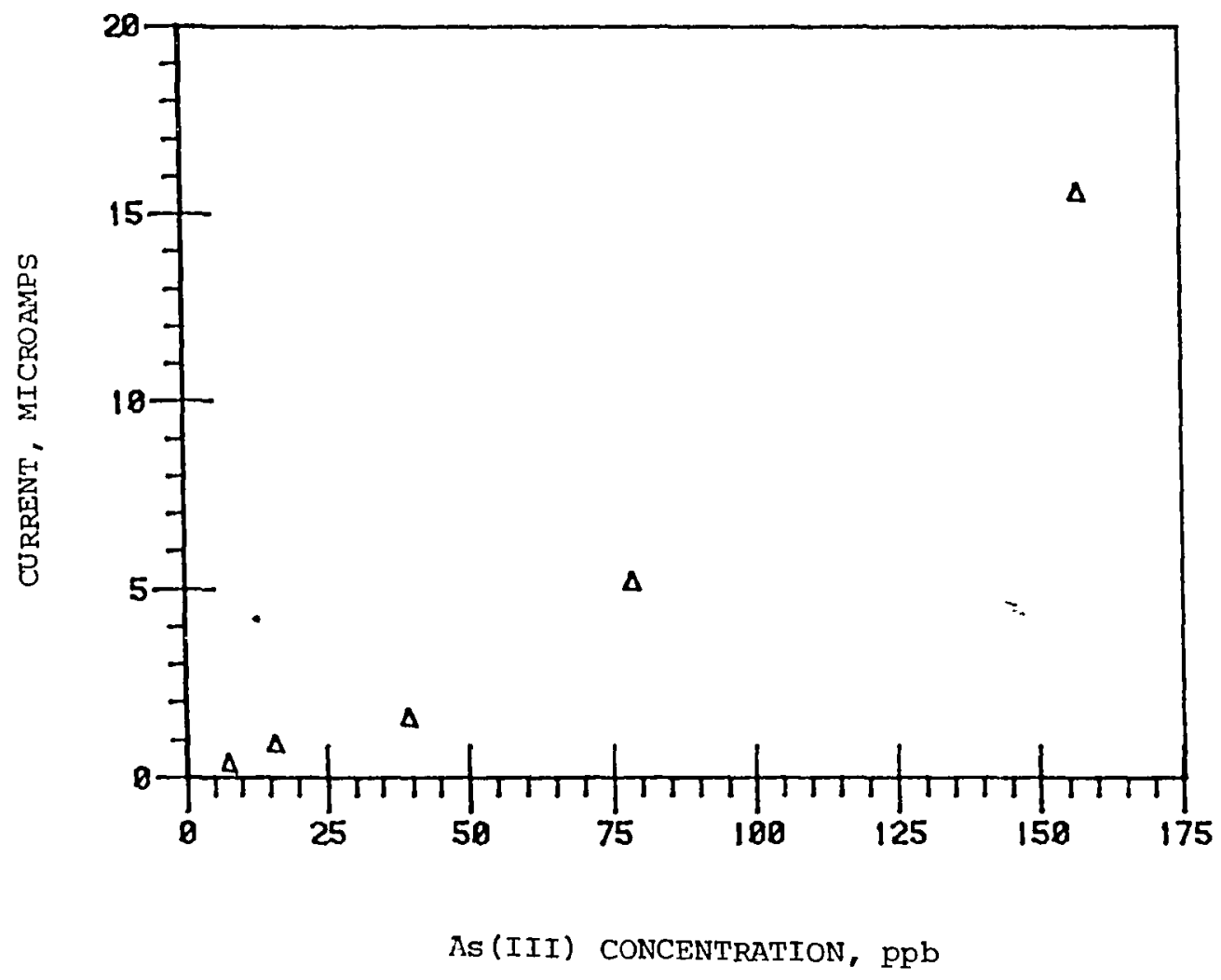

Figure 28. Calibration curve for As(III), step I results.

Detrmined $w_{y}$ differential pulse cathodic stripping voltammetry at a hanging mercury drop electrode. Depostion potential $=-0.4 \mathrm{~V}$. Deposition period $=30 \mathrm{~s}$ stir/ $30 \mathrm{~s}$ quiet Scan rate $=10 \mathrm{mV} / \mathrm{s}$, pulse height $=50 \mathrm{mV}$ $8 \mathrm{ppm} \mathrm{Cu}(\mathrm{I})$ and $1.0 \mathrm{~N} \mathrm{HCl}$. 
catechol and forms the electroactive complex which is measured by cathodic stripping voltammetry. Of course the As(III) is still present in the solution and will contribute to the cathodic stripping signal measured in step II. Experiments were conducted on As(III) solutions to evaluate the As(III) contribution to the cathodic stripping peak in step II. Each As(III) solution was analyzed by the two step procedure. In Figure 28 and 29 the peak current measured for each As(III) solution in experimental steps $I$ and $I I$ is plotted against the As(III) concentration. The As(III) concentration is diluted by $48 \%$ in step II, resulting in a decrease in stripping peak current.

Solutions of $A s(V)$ were also analyzed by the two step procedure. For solutions of $A s(V)$ alone, no stripping peak was observed in the first step of the speciation procedure. Then the catechol solution was added in step II and periodic cathodic stripping experiments were conducted on the As(V) solution until kinetic equilibrium was reached. In Figure 30 the results are presented for one of the stripping experiments conducted on a solution of $667 \mathrm{ppb} A s(V)$. When the decay of the electroactive complex is monitored by cathodic stripping voltammetry only a few analyses can be conducted before equilibrium is reached. In Figure 30 it can be seen that the value of the current-time intercept is not significantly different from the value of the stripping peak current recorded for the first cathodic stripping analysis conducted. The decay of the EAC as monitored by cathodic stripping is too rapid for collection of many data points before equilibrium is reached. Therefore, the stripping current produced from the first deposition period applied at $t=30$ seconds was chosen as the current 


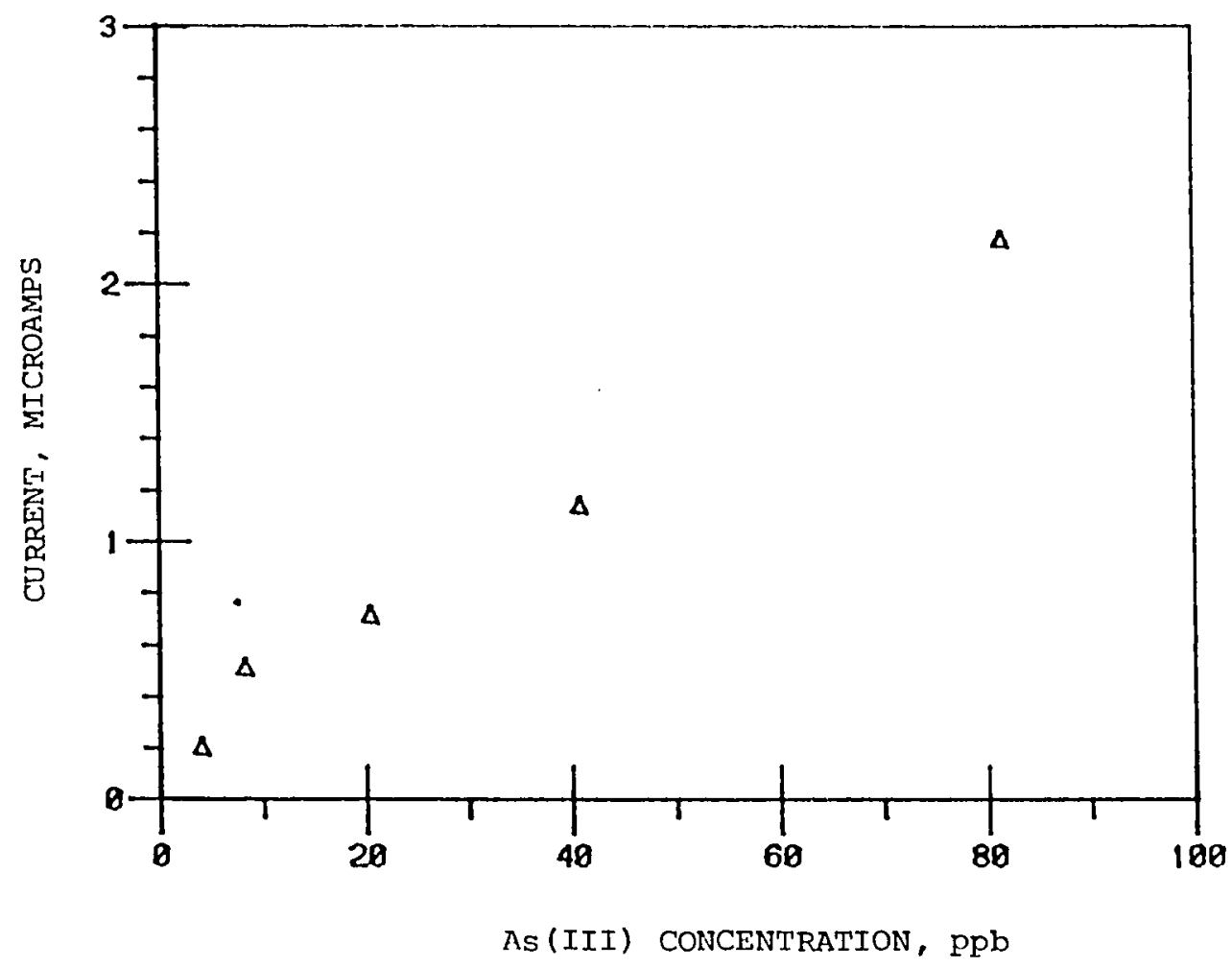

Figure 29. Calibration curve for As (III) as determined by the two step method for inorganic arsenic speciation. Step II results. Determined by differential pulse cathodic stripping voltammetry at a hanging mercury drop electrode. Deposition potential $=-0.4 \mathrm{~V}$. Deposition period $=30 \mathrm{~s}$ stir $/ 30 \mathrm{~s}$ quiet Scan rate $=10 \mathrm{mV} / \mathrm{s}$, pulse height $=50 \mathrm{mV}$ $8 \mathrm{ppm} \mathrm{Cu}(\mathrm{I}), 1.0 \mathrm{~N} \mathrm{HCl}$, and $0.5 \mathrm{M}$ catechol. 


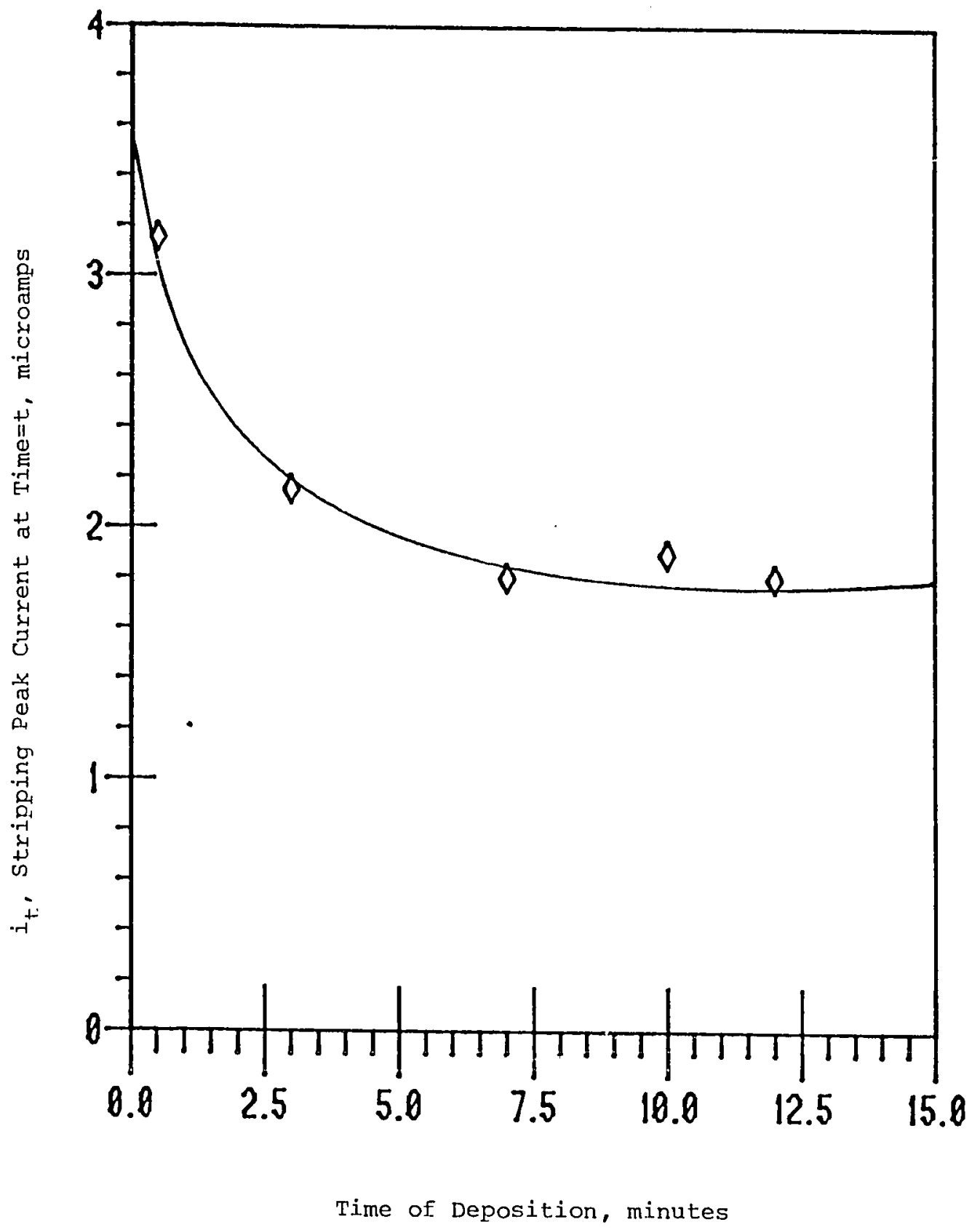

Figure 30. Cuxrent decay of the EAC monitored by cathodic stripping voltammetry with copper codeposition. Solution conditions $=667 \mathrm{ppm}$ As (V), $0.5 \mathrm{M} \mathrm{catechol,} 1.0 \mathrm{~N} \mathrm{HCl}$, and $8 \mathrm{ppm} \mathrm{Cu}(\mathrm{I})$. Deposition potential $=-0.4 \mathrm{~V}$. - Deposition period $=1$ minute. Hanging mercury drop electrode. 
value proportional to As(V) concentration.

Each solution of As(V) was analyzed in a similar manner. Data for each As(V) solution was obtained from the cathodic stripping experiments performed exactly 30 seconds after mixing the components of step II. A calibration curve was then prepared by plotting the step II stripping peak current versus $A s(V)$ concentration, as seen in Figure 31. At low concentrations, the stripping current measured in step II is directly proportional to the As(V) concentration. As the As(V) level increases above $300 \mathrm{ppb}$ the relationship between stripping current and As(V) concentration is no longer linear.

Mixtures of $A s(I I I)$ and $A s(V)$ were analyzed by the same method. A series of experiments were conducted on solutions of constant As(V) concentrations with varied As(III) levels. A different series of experiments was also conducted on solutions of constant As(III) concentration and variable As(V) levels.

The results obtained from experiments conducted on mixtures of 667 ppb As(V) and variable concentrations of As(III) are presented in Figures 32 and 33. In Figure 32 the results of step I analysis are plotted versus the As(III) concentration. The cathodic stripping peak currents measured are linear with As(III) concentration in the range of 0 to approximately $200 \mathrm{ppb}$. The calculated detection limit at the $95 \%$ confidence level is $8.2 \mathrm{ppb}$. The step II cathodic stripping current was also measured for "these solutions of As(III) and As(V). The stripping peak current in step II is the sum of $A s($ III) and As(V) contributions. The As(III) contribution to the step II stripping peak current can be predicted from the calibration curves in Figures 28 and 


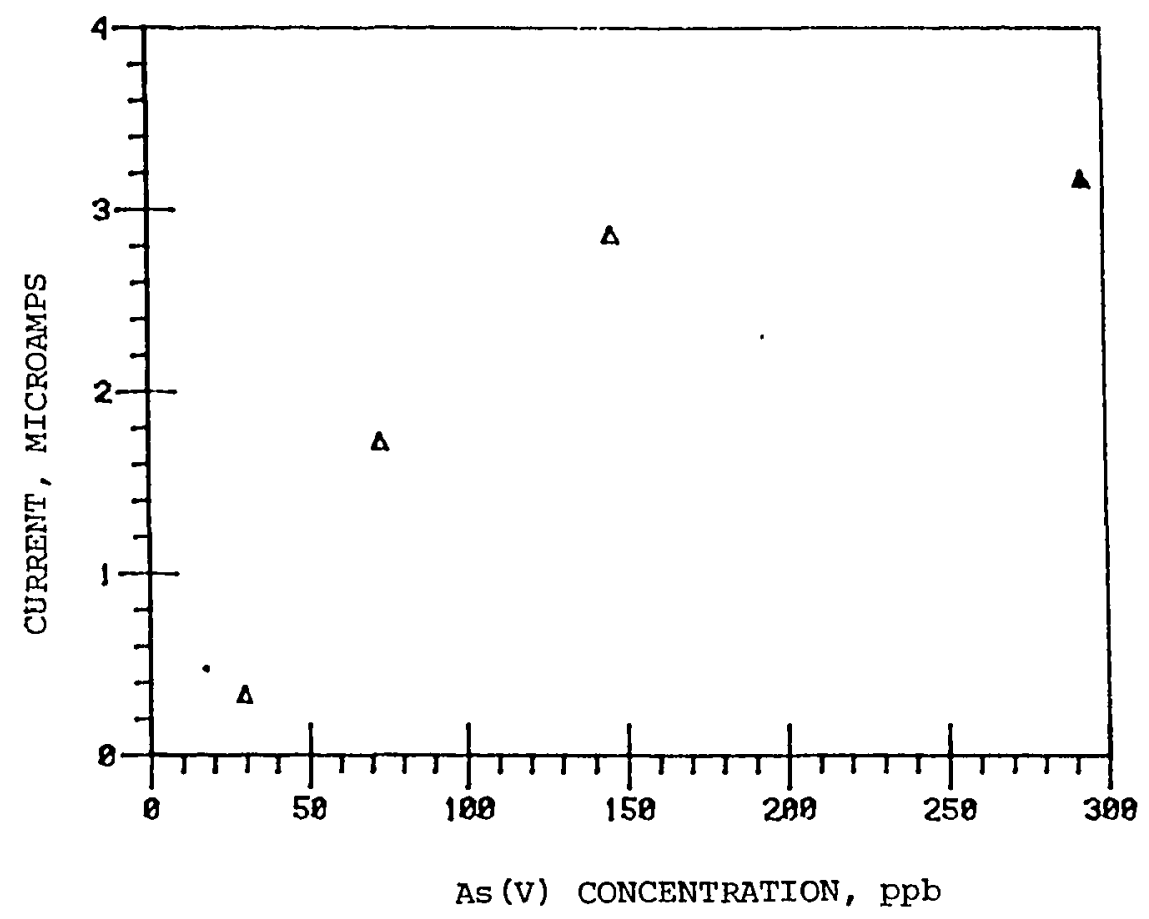

Figure 31. Calibration curve for As(V) determined

by the I'wo step Method for arsenlc speciation. Step II results. Deternined by differential pulse cathodic stripping voltammetry at a hanging mercury drop electrocic. Deposition potential $=-0.4 \mathrm{~V}$. Depostion period $=30 \mathrm{~s}$ stir/ $30 \mathrm{~s}$ quiet Scan rate $=10 \mathrm{mV} / \mathrm{s}$, pulse height $=50 \mathrm{mV}$ $8 \mathrm{ppm} \mathrm{Cu}(\mathrm{I}), 1.0 \mathrm{~N} \mathrm{HCl}$, and $0.5 \mathrm{~m}$ catechol. 


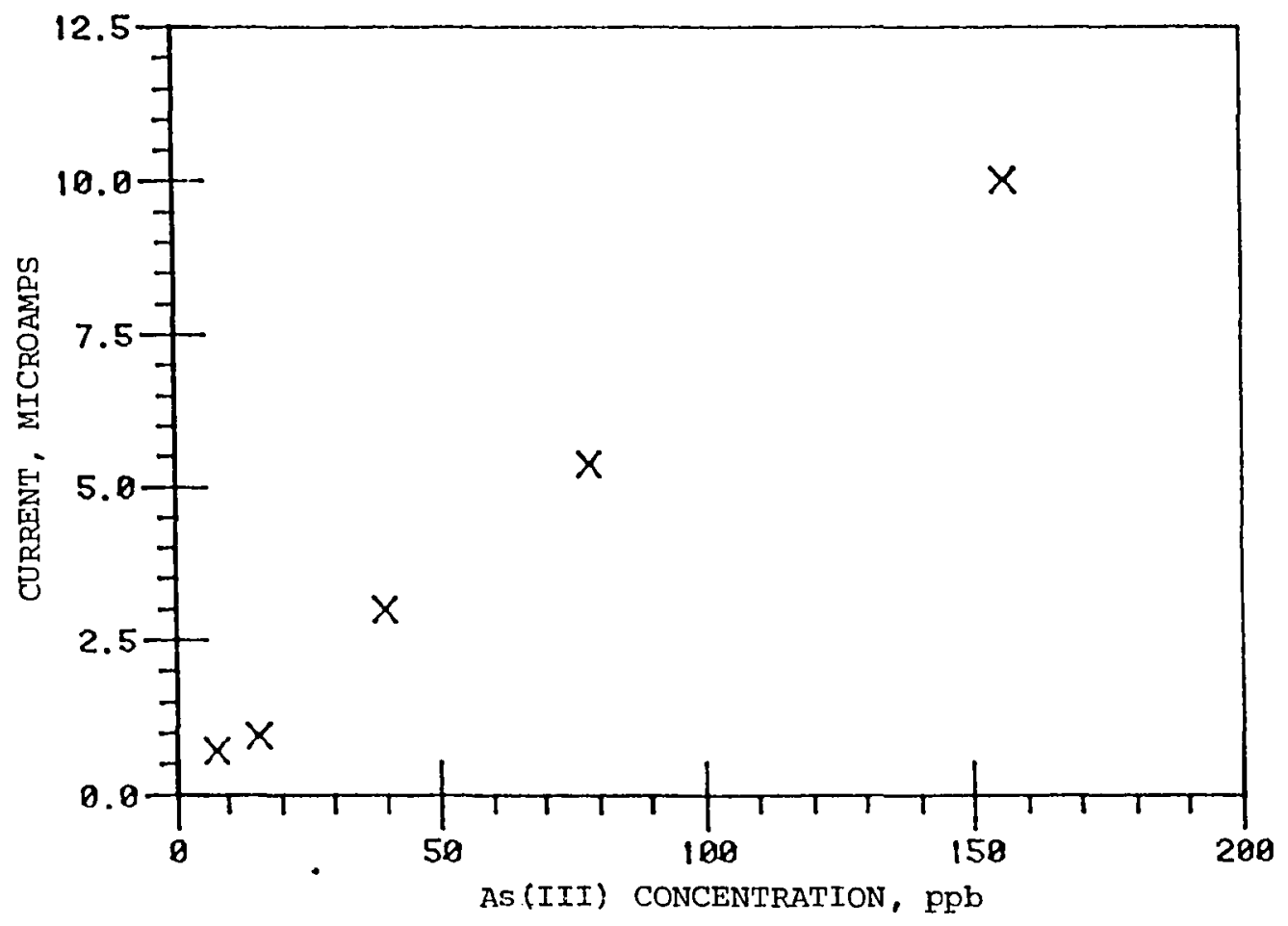

Figure 32. Calibration curve for inorganic arsenic mixture. As $(V)=667 \mathrm{ppb}$. Step I results. Determined by differntial pulse cathodic strif rind voltammetry at a hanging mercury drop electrode. Deposition potential $=-0.4 \mathrm{~V}$. Deposition period $=30 \mathrm{~s}$ stir/ $30 \mathrm{~s}$ quiet Scan rate $=10 \mathrm{mV} / \mathrm{s}$, pulse helght $=50 \mathrm{mV}$ $8 \mathrm{ppm} \mathrm{Cu}(\mathrm{I}), 1.0 \mathrm{~N} \mathrm{HCl}$ 


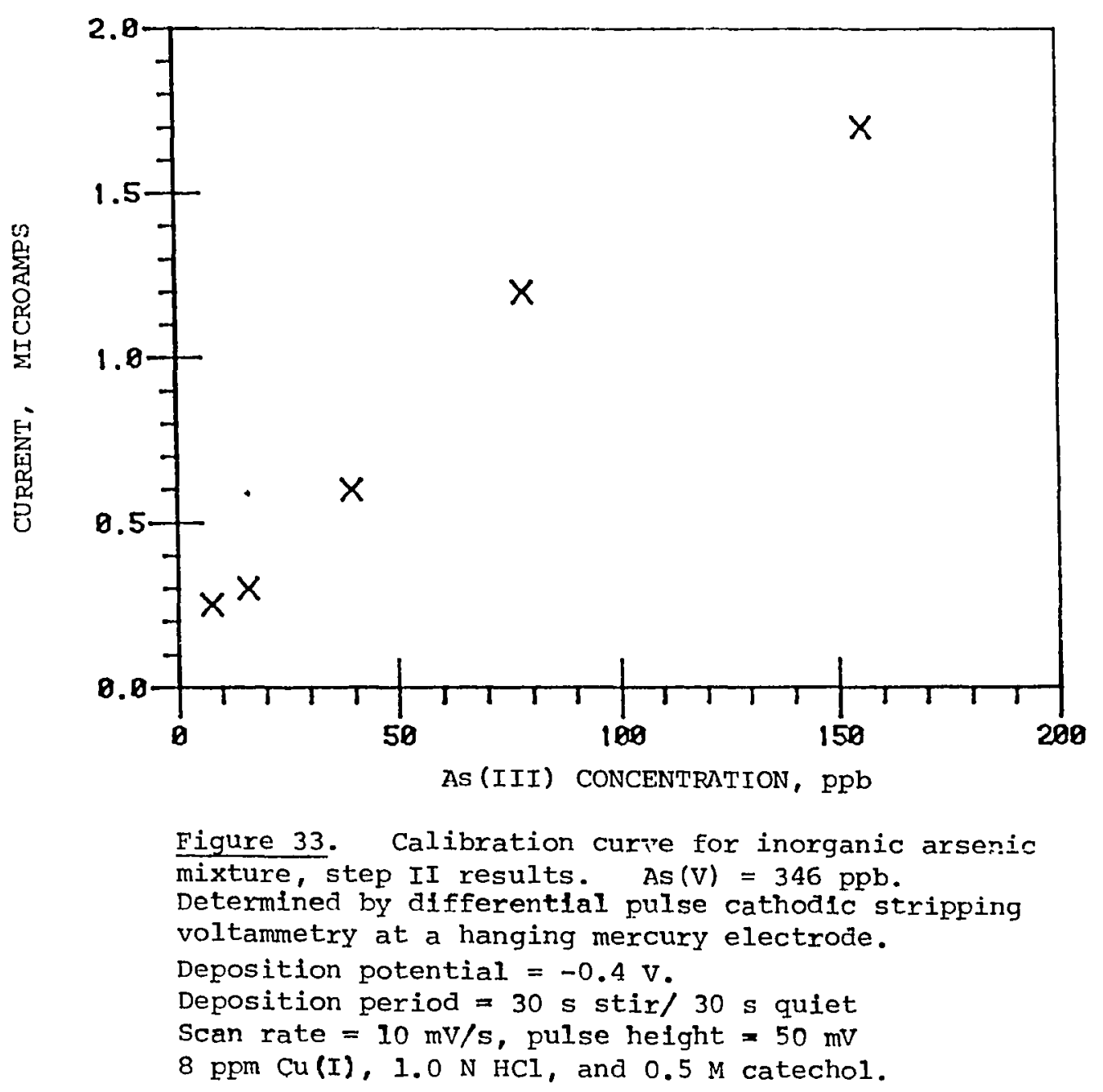


29 and from the step I results for each sample solution. Figure 28 and the stripping peak current measured from step I are used to determine the As(III) concentration. This As(III) concentration is then multiplied by the factor 0.52 yielding the As(III) concentration following step II dilution. Figure 29 and the step II concentration are then used to determine the stripping peak current contributed by As(III) in step II. The As(III) current in step II will be referred to as the

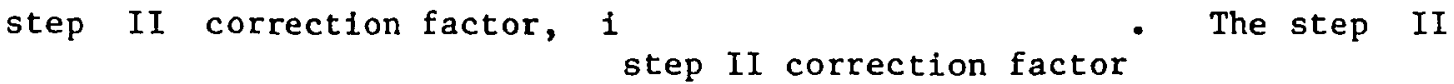

correction factor is subtracted from the step II stripping peak current to give the As(V) stripping peak current, ${ }_{A s(V)}$, as seen in equation 11.

$$
i_{\text {As }(V)}=i_{\text {step II }}-i
$$

The As(V) current is then compared to the As(V) calibration curve, Figure 31 , to determine the As(V) concentration. This subtraction technique is used for all inorganic arsenic determinations. In Figure 34 the $A s(V)$ currents, $i$ As $(V)$, are presented. It can be seen that the series of solutions analyzed for Figure 34 have constant As(V) concentrations and variable As(III) levels.

A second series of experiments were conducted on solutions of constant As(III) concentration of $1.04 \mathrm{uM}, 78 \mathrm{ppb}$, and variable As(V) levels. The peak current for step $I$ is a constant, equal to $31 \mathrm{uA}$, ( standard deviation $=0.36 \mathrm{uA}$, relative standard deviation $=11.6 \%$ ) This is expected since the As(III) concentration is a constant value of $78 \mathrm{ppb}$. In Figure 35 a graph of step II peak current vs. As(V) 


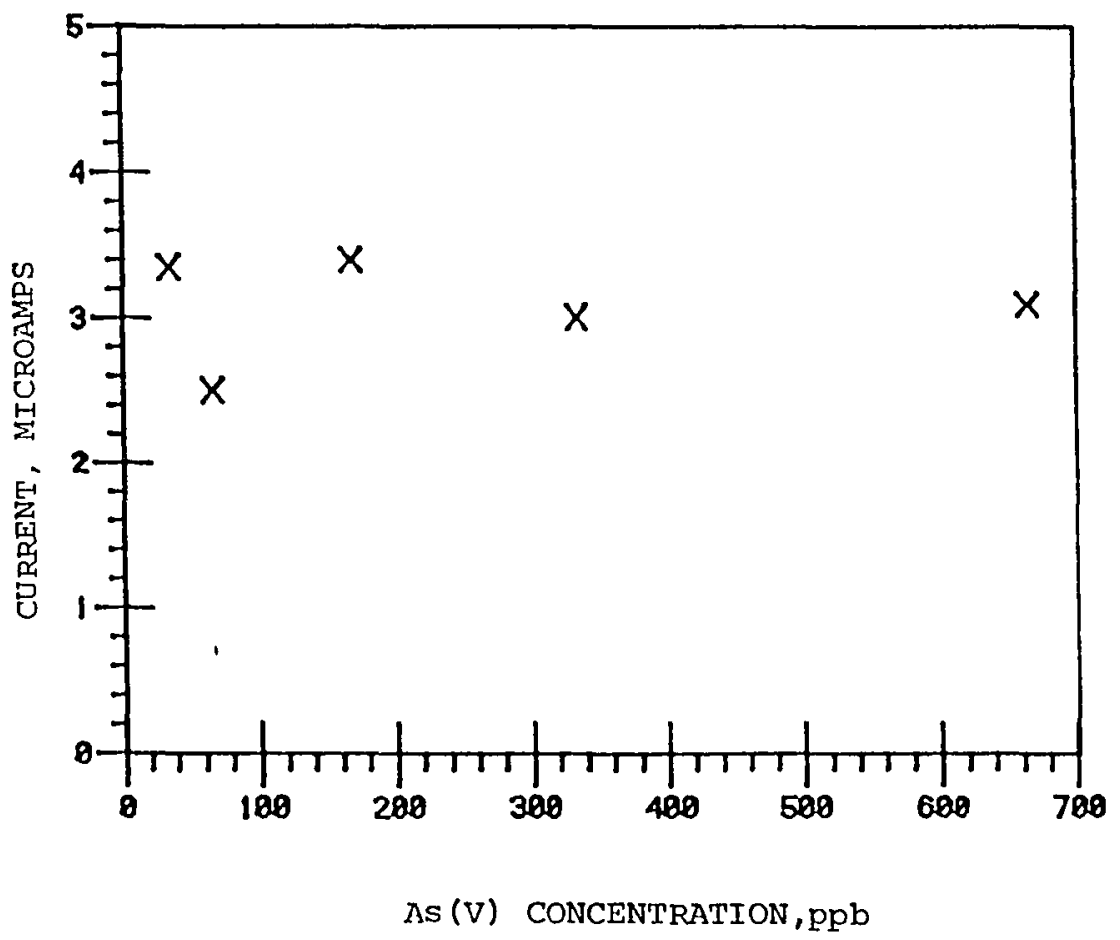

Figure 34. Calibration curve for inorganic arsenic mixture, step I results. As (III) $=78 \mathrm{ppb}$.

Determined by differntial pulse cathodic stripping voltammetry at a hanging mercury drop electrode. Deposition potential $=-0.4 \mathrm{~V}$. Deposition period $=30 \mathrm{~s}$ stir/ $30 \mathrm{~s}$ quiet Scan rate $=10 \mathrm{mV} / \mathrm{s}$, pulse height $=50 \mathrm{mV}$ $8 \mathrm{ppm} \mathrm{Cu}(\mathrm{I})$ and $1.0 \mathrm{~N} \mathrm{HCl}$. 


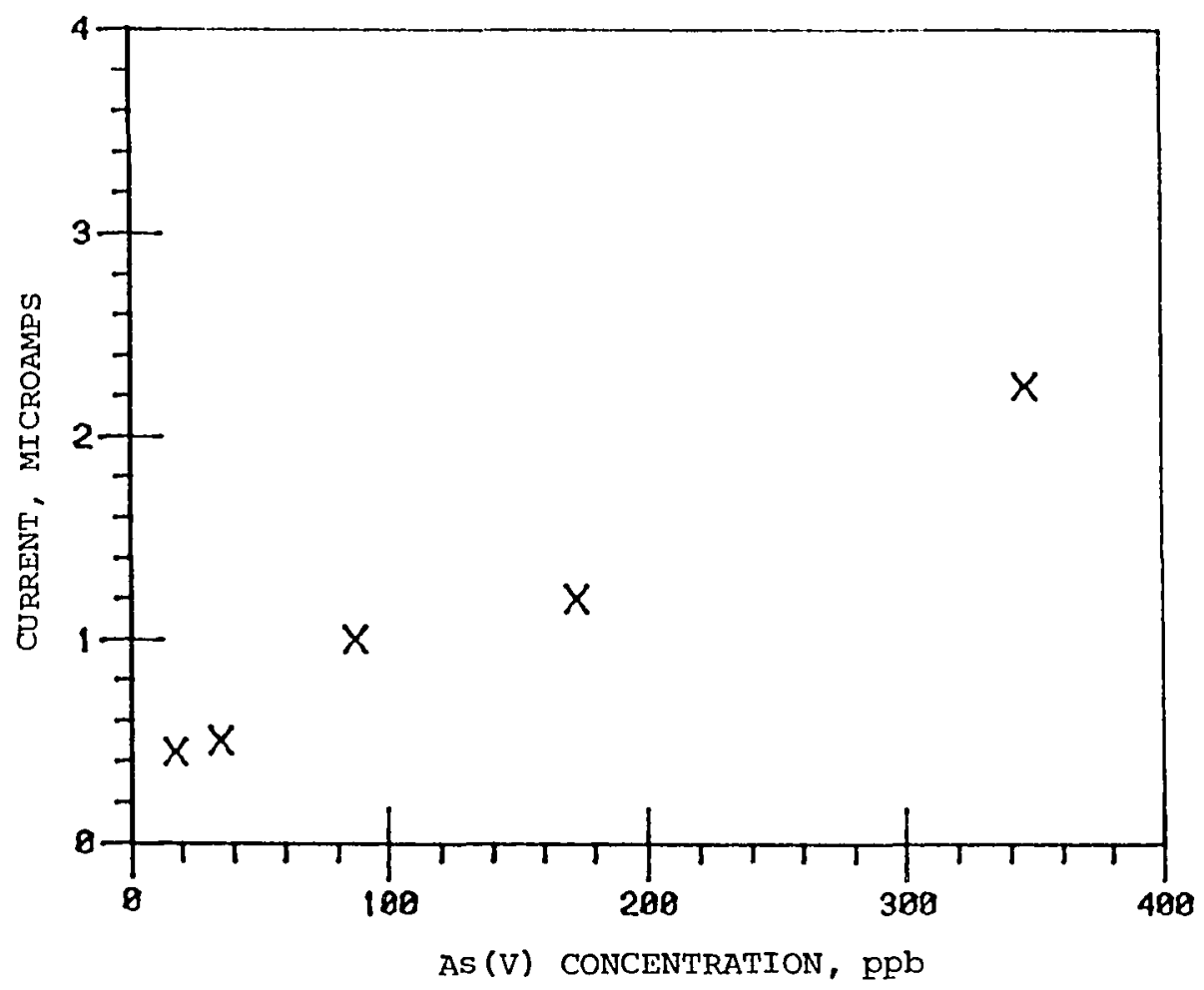

Figure 35. Calibration curve for inorganic arsenic mixture, step II results. As (III) $=37.4 \mathrm{ppb}$. Determined by differential pulse cathodic strlpping voltammetry at a hanging mercury drop electrode. Deposition potential $=-0.4 \mathrm{~V}$. Deposition period $=30 \mathrm{~s}$ stir/ $30 \mathrm{~s}$ quiet. Scan rate $=10 \mathrm{mV} / \mathrm{s}$, pulse height $=50 \mathrm{mV}$ $8 \mathrm{ppm} \cdot \mathrm{Cu}(\mathrm{I}), 1.0 \mathrm{~N} \mathrm{HCl}$, and $0.5 \mathrm{M}$ catechol. 
concentration is presented. The data symbolized by the triangles represents the corrected step II current of As(V) only, i ${ }_{\text {As (V) }}$

The analysis of solutions of As(III) and As(V) illustrate the application of this methou for speciation of inorganic arsenic. 
DISCUSSION

The electrochemical behavior of As(V)-catechol complexes was studied in order to develop an electrochemical technique for measurement of As(V) in water samples. The results of experiments described in the previous chapter will be discussed under the headings of Electroactive Complex Studies, Differential Pulse Polarographic Studies of As(III) and As(V) in the Presence of Catechol and Stripping Vol tammetry.

ELECTROACTIVE COMPLEX STUDIES

Arsenic (V) forms a series of complexes with catechol. These complexes have been studied by a number of researchers. Haak (47) has determined the formation constants for each of the three As(V)-catechol complexes. The formation constants are $0.01,0.02$ and 7.5 for the $1: 1$, $1: 2$, and $1: 3$ complexes respectively. In equilibrium solutions of $\mathrm{pH}<2$, excess catechol and As(V) the predominant solution species is the $1: 3$ complex.

The electrochemical behavior of the solid 1:3 complex was also reported by Haak (47). Small samples of the $1: 3$ complex $(0.012$ g) were dissolved in $1 \mathrm{~N} \mathrm{NaClO}$ and monitored by polarography. A reduction wave 4

was observed which decayed with time. The reduction current disappears as dilute solutions $(1 \mathrm{mM})$ of the $1: 3$ complex reach equilibrium after dissolution of the solid complex. This corresponds to the complete dissociation of the $1: 3$ complex to form As(V) and catechol.

In preliminary experiments a fresh mixture of As(V), excess 
catechol and supporting electrolyte produced a reducible species whose concentration is a maximum at time of initial mixing and then decays to reach an equilibrium value. In solutions of low As(V) concentration, $0.2 \mathrm{mM},(15.0 \mathrm{ppm}), \mathrm{pH}=1$, and high catechol concentration, $0.5 \mathrm{M}$, the decay of the electroactive species is observed. These conditions result in an equilibrium solution in which the predominant arsenic species is the $1: 3$ As(V)-catechol complex. At kinetic equilibrium the electroactive complex is at a minimum level and the $1: 3$ complex is the predominant species.

The reactions occurring in solutions of $\mathrm{As}(\mathrm{V})$ and catechol prior to equilibrium include the stepwise formation of the $1: 1$ and $1: 2$ complexes. One or both of these complexes must be the electroactive species. This conclusion is also supported by the dissolution experiments conducted with the solid $1: 3$ complex by Haak (47). As the $1: 3$ complex dissociates completely it must form the $1: 2$ and $1: 1$ complexes as intermediates prior to the formation of uncomplexed As(V) and catechol. One of the lower order As(V)-catechol compleas is the electroactive species whose transient existance can be montored by electrochemical techniques.

The currents measured for reduction of the electroactive complex are proportional to the $A s(V)$ concentration and can be used to quantify the $A s(V)$ concentration in analytical samples. Studies of the electroactive complex were conducted to identify which of the complexes are electroactive and to find the optimum conditions for measurement of the electroactive complex concentration.

The As(V)-catechol system is very complex. Two reaction regimes 
must be considered in the study of the EAC. The first regime to consider is the formation of the EAC. In equation 12 the general reaction of $A s(V)$ and catechol to form the EAC is presented.

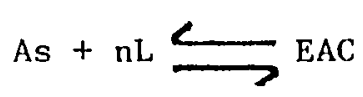

$$
\mathrm{n}=1 \text { or } 2
$$

The symbol $\mathrm{L}$ represents catechol, the 1 igand, which reacts with As (V) to form the EAC, which may be of $1: 1$ or $1: 2$ stoichiometry. The second reaction regime to consider is the decay of the EAC to form the predominant, electroinactive $1: 3 \mathrm{As}(\mathrm{V})$-catechol complex at equilibrium. In equation 13 the general reaction equation for the decay of the EAC is presented.

$$
\begin{aligned}
& \mathrm{EAC}+\mathrm{nL} \coprod_{3} \mathrm{AsI}_{3} \\
& \mathrm{n}=2 \text { or } 1
\end{aligned}
$$

The first regime, formation of the EAC, can be examined using the kinetic decay data collected for solutions of different catechol concentrations. The values of $i$ calculated from the kinetic decay data can be used to calculate equilibrium constants through application of the appropriate working curve developed by Nicholson and Shain(52) for linear scan voltammetry. The general reaction mechanism of the EAC must be identified before the working curves of Nicholson and Shain (52) can be applied. The reduction of the EAC does not occur by just a simple heterogeneous electron transfer reaction. Examination of the 
current voltage curves recorded for linear potential scan experiments of the EAC reveals clues to its reduction mechanism. The general mechanism for reduction of the EAC can be identified by comparison of the $\mathrm{CV}$ curves recorded for the EAC as seen in Figure 6 to the CV curves calculated by Nicholson and Shain (54). The CV curves for the EAC are essentially identical to the CV curves calculated by Nicholson and Shain (52) for a CE electrode reaction mechanism. The abbreviation CE stands for a two step mechanism composed of a rate limiting chemical step followed by an electron transfer step. The reduction of the EAC can be further described as a CE mechanism, where the subscript $i$ denotes an i irreversible electron transfer step. The irreversible nature of the EAC reduction can be observed in Figure 5 where the oxidation of the electrode reduction products is not observed in the anodic portion of the cyclic scan. In equations 14 and 15 the general mechanism is shown.

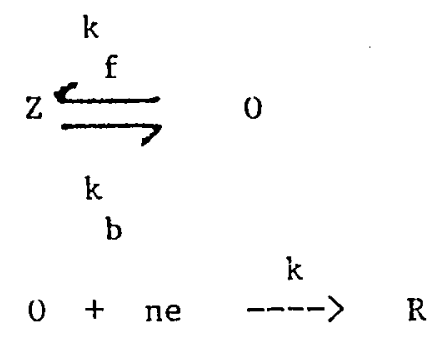

The symbol $\mathrm{Z}$ represents the solution phase species in equilibrium with the EAC, represented by 0 . At fast scan rates, $200 \mathrm{mV} / \mathrm{s}$, the influence of the slow chemical step on the CV curve, equation 13, is not significant and a classical linear scan reduction peak is observed. At slow scan rates, such as $10 \mathrm{mV} / \mathrm{s}$, the chemical step has significant influence on the shape of the $\mathrm{CV}$ curve producing a current rise followed by a current plateau. The working curves developed by Nicholson and 
Shain (52) were prepared from solution of the empirical equation presented below.

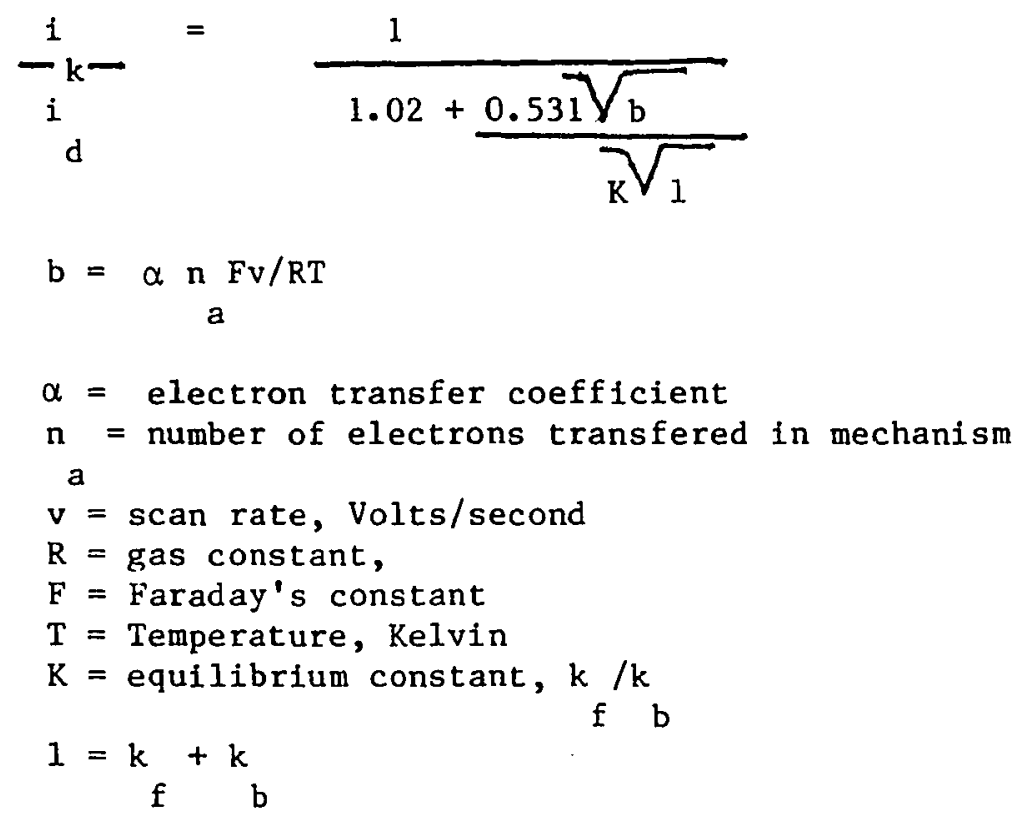

By manipulation of the above equation and use of experimental data, the equilibrium constant for formation of the EAC from As(V) and catechol can be determined. The experimental data to be treated in these calculations consists of kinetic data monitored at a variety of scan rates. Scan rate, or the square root of scan rate, was chosen as the independent variable and equation 16 was then manipulated into a linear form proportional to the square root of scan.rate. In equation 17 the term $b$ has been replace by its components and the square root of scan rate is multiplied by a number of constants.

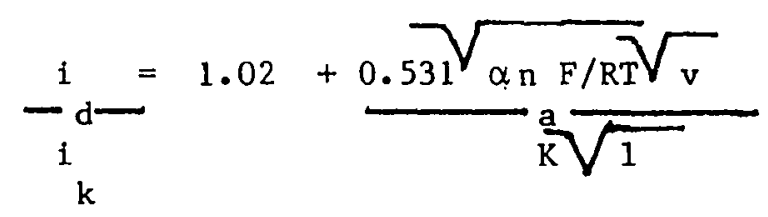


The value of $i$, the kinetic current, is provided from experimental data. The value of the diffusion current, $i$, must be calculated. In general the reduction current is proportional to the square root of the scan rate (51) for faradic processes. This generality is expressed in equation 18 for the diffusion current, 1 .

$$
i_{d}=\vec{p} \sqrt{v}
$$

In these calculations, $P$ is the proportionality constant for the diffusion current. The expression, $\bar{P} \sqrt{v}$, is substituted into equation 17 for the diffusion current, $i$. This substitution is seen in equation 19.

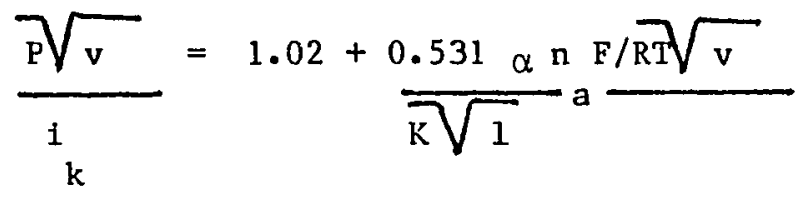

Each s:de of equation 19 is then multiplied by $(1 / \mathrm{P})$ to produce the following result in equation 20.

$$
\underbrace{\sqrt{v}}_{i k}=\frac{1.02}{P}+\frac{0.531 \alpha \mathrm{nF} / \mathrm{RT} \sqrt{v}}{P \mathrm{~K} \sqrt{1}}
$$

This is a linear equation in $\sqrt{v}$. When the value of $\sqrt{v} / i$ is plotted as a function of $\sqrt{v}$. The intercept, $1.02 / \mathrm{p}$, can be used to calculate the value of $P$, the proportionality constant for the diffusion current. With the value of $P$ the diffusion current, 1 , can be calculated using equation 18.

The value of $P$ is then calculated from the kinetic data collected 
experiments conducted at a variety of catechol concentraticns. Calculations were performed on current data where $i$ was chosen as the value for $i$

$\mathrm{k}$

The calculated difiusion current, determined with equation 18 , and the kinecic current, measured from experimental data, can be used to determine the equilibrium constant for equation 14 . In equation 21 the equilibrium expression is presented for the chemical reaction in equation 14.

$$
K=\frac{[0]}{[2]}=\frac{k}{k} \underset{b}{k}
$$

The diffusion limited current, $i$, is proportional to the concentration of species 0 and $z$.

$$
i \underset{d}{i}\{[0]+[z]\}
$$

The kinetic current, $i$, is proportional to the concentration of 0 plus the fraction of species $Z$ which converts to species 0 during the course of the linear potential scan. In equation 23 the proportionality for the kinetic current is presented.

$$
\underset{k}{i} \propto\left\{[0]+\frac{[z]}{v}\right\}
$$

Equations 22 and 23 are then used to prepare a ratio of $i_{k}$ to $i_{d}$ as seen in equation 24. 


$$
{\underset{i}{d}-\infty}_{[0]+[z]}^{[0]+[z] / \sqrt{v}}
$$

This equation can be rearranged into a form suitable for graphing.

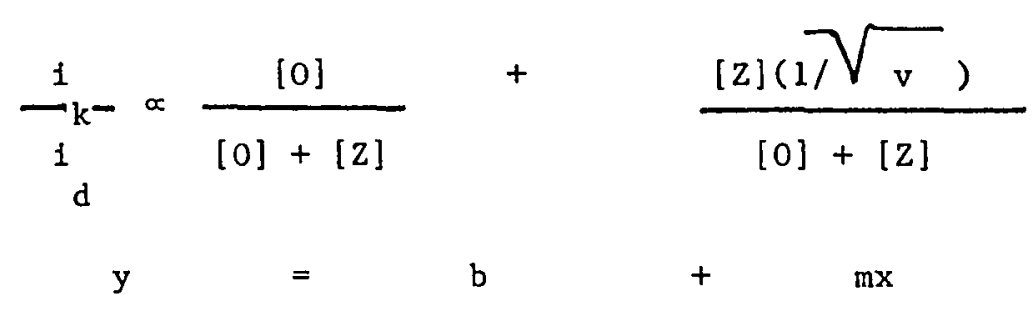

Equation 26 is plotted by graphing the ration of $1 / i$ versus $1 / \sqrt{v}$. The values of slope, $[z] /([0]+[z])$, and intercept, $[0] /([0]+[z])$, can be used to calculate the equilibrium constant for equation 20. This calculation is seen in equation 26 below.

$$
K=\frac{\text { int }}{\text { slope }}=\frac{[0]}{\frac{[0]+[z]}{[z]}}=\frac{[0]}{[z]}
$$

In Figure 36 equation 25 is plotted for kinetic experiments conducted on $A s(V)$ and catechol solutions in $0.1 \mathrm{~N} \mathrm{HCl}$. Figure 37 presents similar data for kinetic experiments conducted in $1.0 \mathrm{~N} H C 1$. Table III presents the results of calculations performed using the above equations. The slope, intercept and correlation coefficient for each line found in Figures 36 and 37 are found in Table III. The equilibrium constant, $K$, calculated from the above equations corresponds to the simple first order reaction of the CE mechanism, equation 14 . The equilibrium expression for equation $14^{1}$ is shown below. 


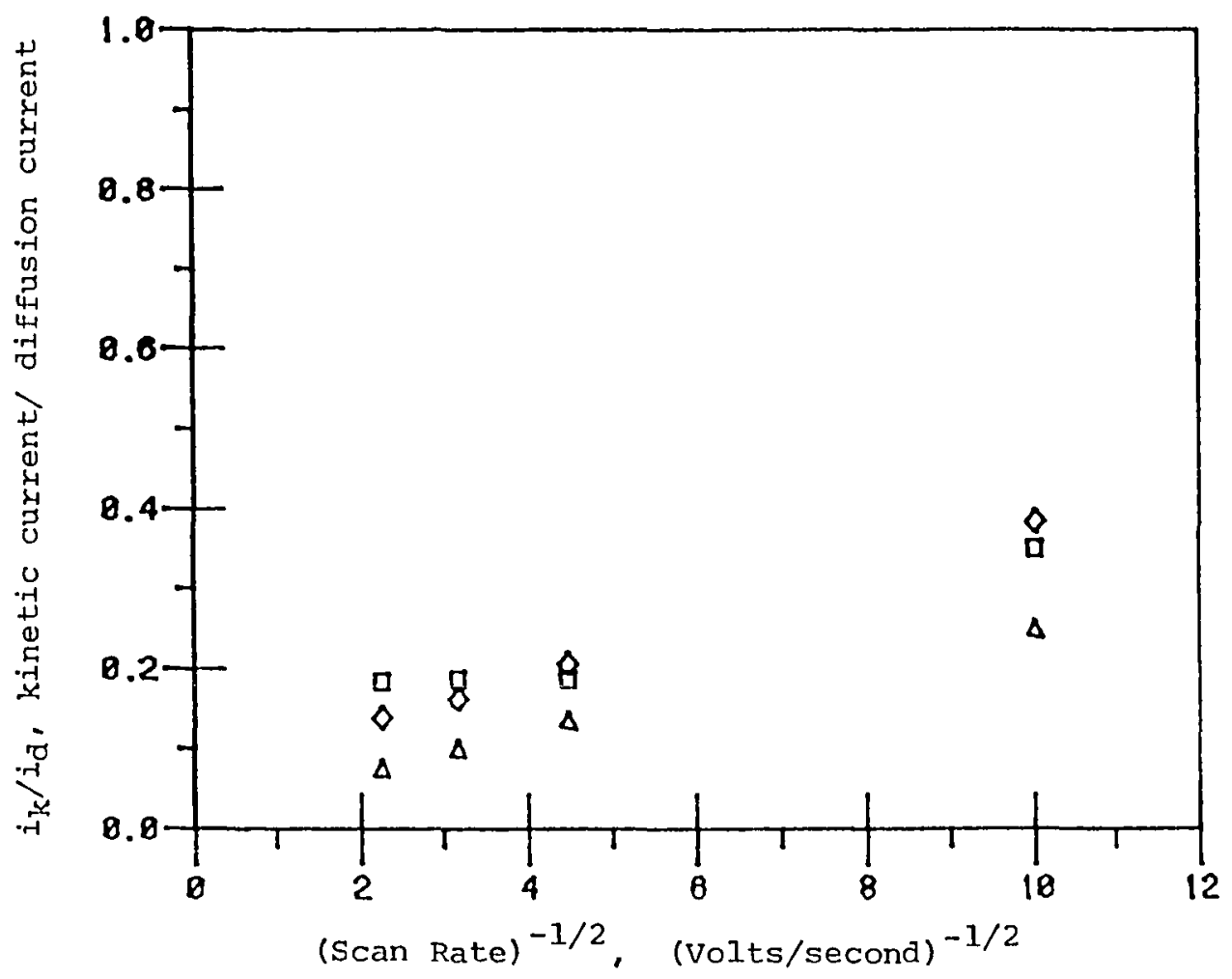

Figure 36. Ratio of kinetic current to diffusion current as a function of the inverse of the square root of scan rate, experiments conducted in $0.1 \mathrm{~N}$ $\mathrm{HCl}$. $1.25 \mathrm{mM}, 94 \mathrm{ppm} \mathrm{As}(\mathrm{V})$.

triangle: $0.262 \mathrm{M}$ catechol diamond: $0.491 \mathrm{M}$ catechol square: $0.695 \mathrm{M}$ catechol Data from linear potential scan experiments performed at a hanging mercury electrode. 


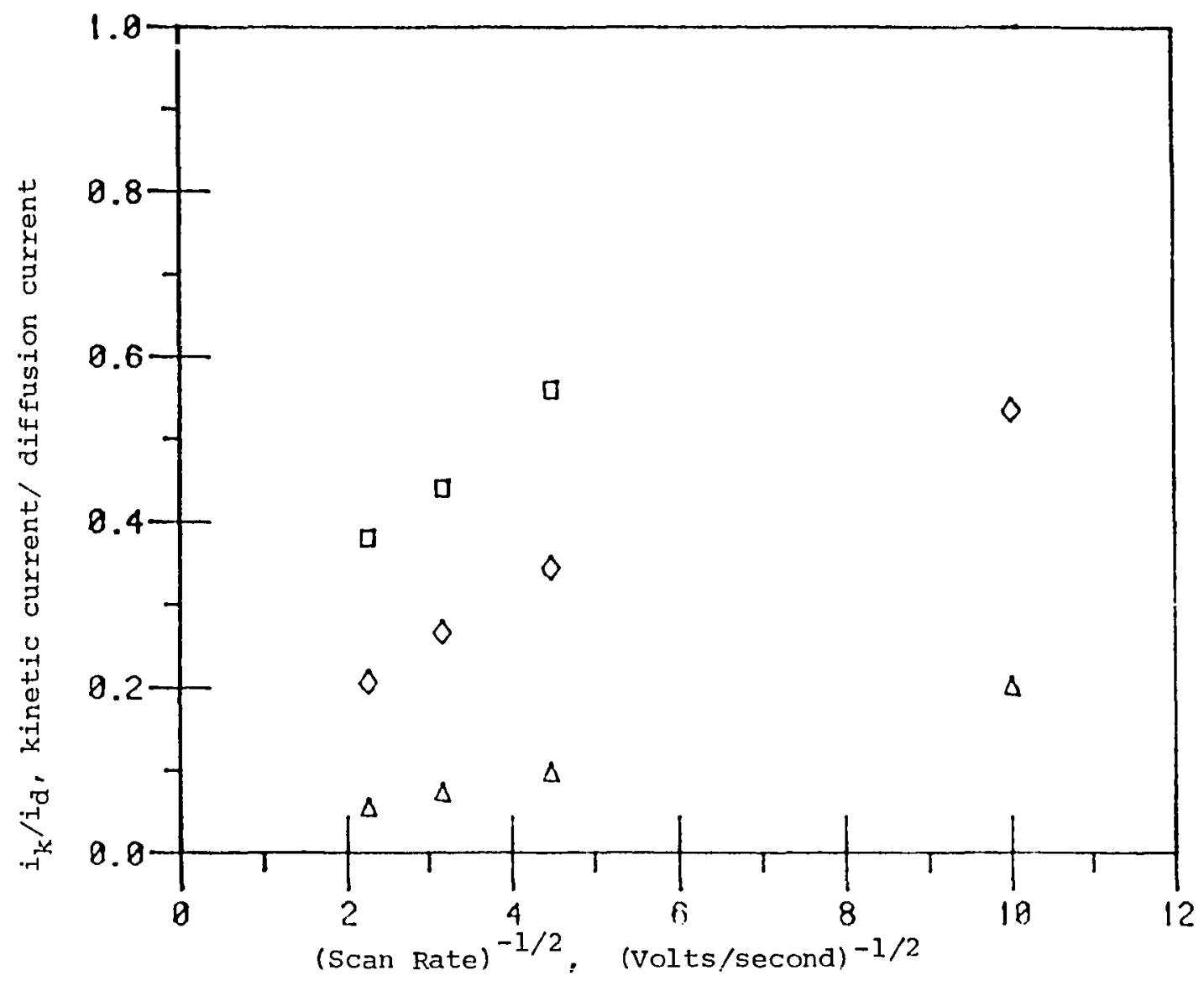

Figure 37. Ratio of kinetic current to diffusion current as a function of the inverse of the square root of scan rate, experiments conducted in $1.0 \mathrm{~N} \mathrm{HCl} .1 .25 \mathrm{mM}, 94 \mathrm{ppm} \mathrm{As}(\mathrm{V})$.

triangle: $0.249 \mathrm{M}$ catechol diamond: $0.492 \mathrm{M}$ catechol square: $0.735 \mathrm{M}$ catechol Data from linear potential scan experiments prformed at a hanging mercury drop electrode. 
In reality the formation of the $\mathrm{EAC}$ is more complex, as seen in equation 12. The equilibrium expression for equation 12 is illustrated below, where the equilibrium constant is referred to as $\mathrm{K}^{\prime}$.

$$
K^{\prime}=\frac{[E A C]}{n}
$$

This expression can be rearranged into a form which is equivalent to the equilibrium expression for the simple first order reaction in equation 14.

$$
K^{\prime}[L]=\frac{[E A C]}{[A s]}=\frac{[0]}{[Z]}=K
$$

The equilibrium constant, $K^{\prime}$, for the second order reaction of arsenic and catechol can be calculated from the following equation (30) and values of $K$.

$$
\mathrm{K}^{\prime}=\underline{\mathrm{K}}_{\mathrm{n}}
$$

In Table III the values of $\mathrm{K}^{\prime}$ are presented for the two possible cases where $n=1$ or $n=2$, as calculated from equation 30 . The values of $\mathrm{K}^{\prime}$ are presented for acid concentrations of $0.1 \mathrm{~N} \mathrm{HCl}$ and $1.0 \mathrm{~N} \mathrm{HCl}$.

\footnotetext{
The average value of $\mathrm{K}^{\prime}, \mathrm{n}=1$, in solutions of $1.0 \mathrm{~N} \mathrm{HCl}$ is 2.67 $-1$ (mol/L) with a relative standard deviation of $16.6 \%$. The average
} 
equilibrium constant, $K^{\prime}, n^{\prime}=1$, for solutions of $0.1 \mathrm{~N} \mathrm{fiCl}$ is 4.02 $-1$

$(\mathrm{mol} / \mathrm{L})$ with a similair relative standard deviation of $16.0 \%$. The values of $\mathrm{K}^{\prime}, \mathrm{n}=1$, calculated for reaction 13 can be compared to the values of $B_{1}, 0.01(\mathrm{~mol} / \mathrm{L})^{-1}$, estimated by Haak (47). The values of $K^{\prime}$ calculated in this study are at least one hundred times greater. The value of $B$ reported by Haak (47) is really "too" small to be reliably 1

estimated using the potentiometric data and regression techniques of Haak (47). Another cause of the difference between the value calculated in this study and the value reported by Haak (47) is the result of the different experimental conditions used. The constants calculated in this study came from experiments conducted at $\mathrm{pH}^{\prime} \mathrm{s}$ of 0 and 1. The experiments performed by Haak were conducted in the $\mathrm{pH}$ range of 2. The difference in $\mathrm{pH}$ conditions may result in the difference in reported equilibrium constants.

The values of $K^{\prime}, n=2$, are presented in table III. The values of $K^{\prime}, \quad n=2$, differ significantly for experiments conducted at differnet catechol concentrations. The mean value of $\mathrm{K}^{\prime}, \mathrm{n}=2$, in $0.1 \mathrm{NHCl}$ is $-2$

$7.51(\mathrm{~mol} / \mathrm{L})$ with a relative standard deviation of $59.9 \%$. The mean value of $\mathrm{K}^{\prime}, \mathrm{n}=2$, calculated for solutions of $1.0 \mathrm{~N} \mathrm{HCl}$ is 11.40 $-2$ $(\mathrm{mol} / \mathrm{L})$ with a relative standard deviation of $60.1 \%$. The size of the standard deviation of the values of $K^{\prime}, n=2$, does not support the claim that the identity of the $\operatorname{EAC}$ is $A s(L)$. The values of $K^{\prime}, n=1$, on the other hand, are consistant and readily identify the EAC as the $1: 1$ As (V)-catechol complex, AsL. 
TABLE III

DATA AND RESULTS OF EQUILIBRIUM CONSTANT CALCULATIONS USING NICHOLSON AND SHAIN DIAGNOSTIC CRITERIA

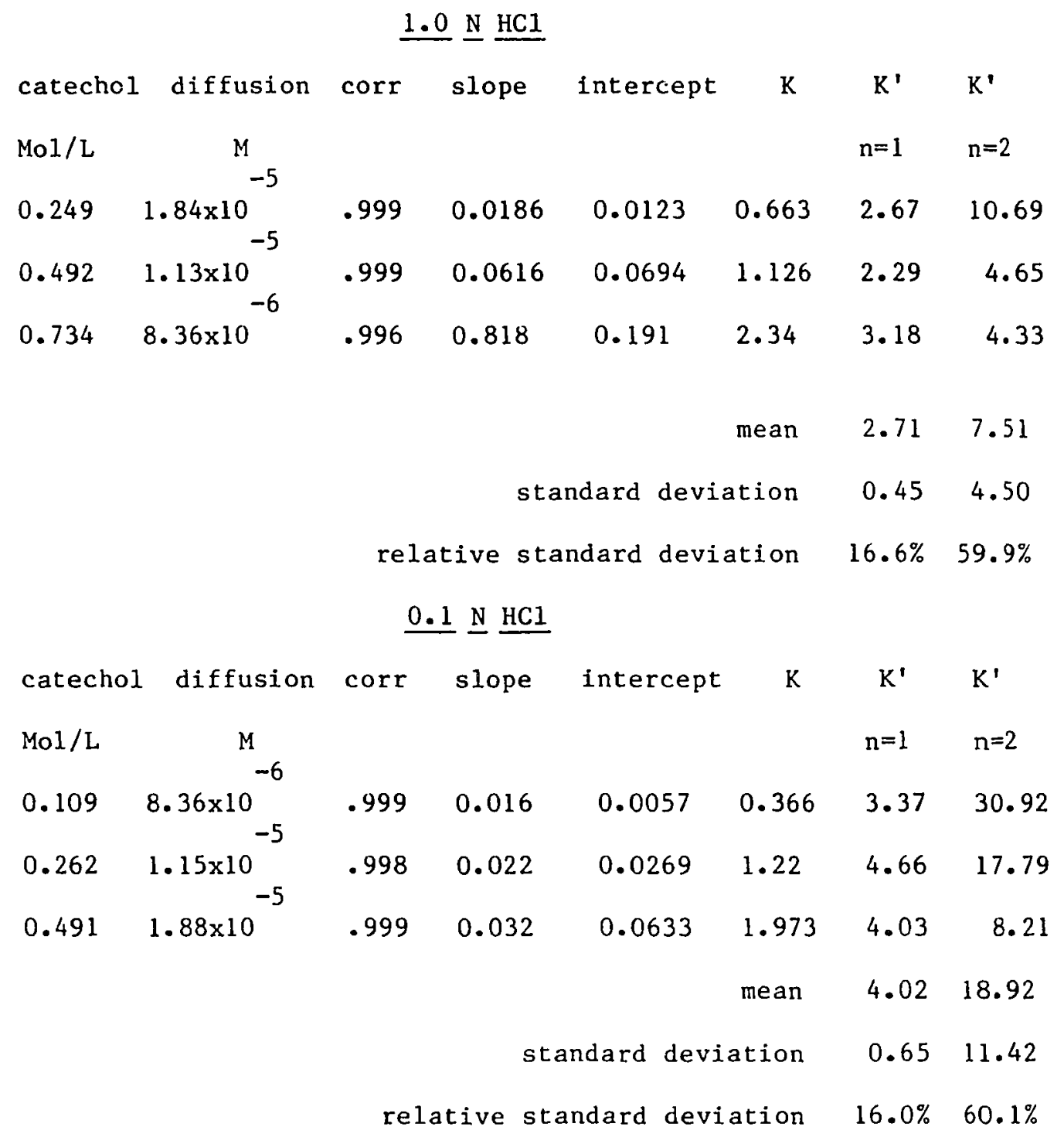

Other evidence also reinforces the conclusion that the $1: 1$ complex is the EAC. In Figures 21 and 22 a plot of the values of $i$ versus catechol concentration is presented for kinetic experiments performed on 
solutions of $0.1 \mathrm{~N} \mathrm{HCl}$ and $2.5 \mathrm{mM} \mathrm{As}(\mathrm{V})$. These are linear functions which level out at higher catechol concentrations. At low catechol concentrations the value of $i$, which is proportional to the EAC concentration, is also directly proportional to catechol concentration. This is additional evidence that the $1: 1$ complex is indeed the EAC.

Hydrogen Ion Dependence

The As(V)-catechol system is very complex. Hydrogen ion plays an important role in the formation and decay of the EAC. The influence of hydrogen ion on the formation and decay of the EAC will be discussed below.

The role of hydrogen ion in the formation of the EAC can be described in terms of reaction behavior observed to occur in two different $\mathrm{pH}$ regimes. In the first regime, $\mathrm{pH}>=2$, the formation of the EAC occurs at a slow rate and can be observed with these electrochemical techniques. Figure 20 is an example of an experiment where the formation of the EAC was slow enough to be monitored by electrochemical methods. The second concentration regime is observed at $\mathrm{pH}$ levels which are less than 1.5. In this $\mathrm{pH}$ regime the formation of the EAC has a fast rate and cannot be observed by electrochemical methods used here. Figure 18 presents an example of an experiment where the EAC is at a maximum level at reaction initiation and only its decay is observed during the kinetic experiment. From this evidence conclusions can be draw about the role of hydrogen ion in the formation of the EAC.

Hydrogen ion plays a role in the formation of the EAC. The 
equilibrium constants calculated for the conditions of $\mathrm{pH}=1$ and $\mathrm{pH}=0$ differ by approximately $33 \%$. This is a small difference in equilibrium constant values for a change in the hydrogen ion concentration of an order of magnitude.

The character of the reactants can be examined in order to understand the role of hydrogen ion in the formation of the EAC. At low $\mathrm{pH}^{\prime} \mathrm{s}, \quad(\mathrm{pH}<=1.5)$, both of the reactants, $\mathrm{As}(\mathrm{V})$ and catechol, are fully protonated. $A s(V)$ and catechol are moderately weak acids. The values of $\mathrm{pKa}$, pKa, and $\mathrm{pKa}$ for arsenic acid are $2.22,6.98$, and 11.50 respectively (55). The values of $\mathrm{pKa}$ and $\mathrm{pKa}$ for catechol are 9.36 and 12.98 (55). In equation 31 the formation of the $\mathrm{EAC}$ is illustrated.

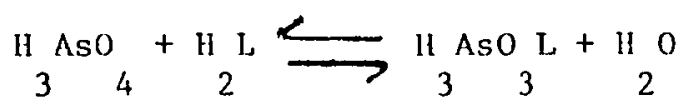

In reaction equation 31 there is not an obvious role for hydrogen ion to play in the formation of the EAC. It may function as an acid catalyst, initiating formation of the EAC by protonation of arsenic acid, Il $\mathrm{AsO}$, to form $\mathrm{HAsO}{ }^{+}$, which may be an important intermediate in the mechanism of FAC formation. Fquations 32 and 33 illustrate two steps which may be involved in the formation of the EAC.

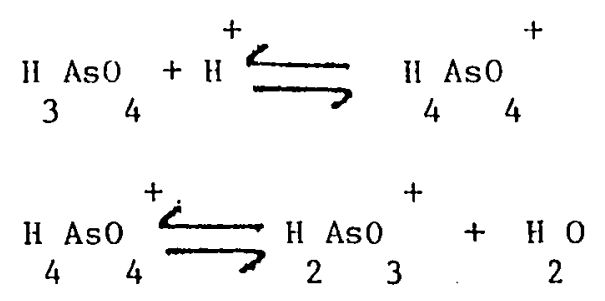

Sufficient details are not available for further speculation on the 
mechanism for formation of the EAC.

When the $\mathrm{pH}$ increases above 2.2 arsenic acid is ionized to form a hydrogen ion and $\mathrm{HAsO}$. When $A s(V)$ is present as this species it may not participate in the reaction to form the EAC, thus decreasing the concentration of reactant and decreasing the reaction rate.

The decay of the electroactive complex is also influenced by hydrogen ion concentration. When the hydrogen ion concentration is low, at $\mathrm{pH}>2$, the decay of the EAC is relatively slow. When the hydrogen ion concentration is high, $\mathrm{pH}<2$, the decay of the EAC is faster. In TABLE IV the data collected for kinetic experiments are presented. The rate of the pseudo first order decay of the EAC is related to the slope of the kinetic decay plots prepared for each experiment. It can be seen that the value of the slope of the kinetic decay plots vary with changes in catechol concentration and pH.

TABLE IV

DATA FROM KINETIC PLOTS OF DECAY EXPERIMENTS CONDUCTED ON SOLUTIONS OF $1.25 \mathrm{mM} \mathrm{As}(\mathrm{V})$

$\underline{0.1} \underline{\mathrm{N}} \underline{\mathrm{HCl}}$

$\begin{array}{cccc}\text { CATECHOL } & \text { SLOPE } & \text { STANDARD } & \text { RELATIVE STANDARD } \\ \text { CONCENTRATION } & & \text { DEVIATION } & \text { DEVIATION } \\ 0.262 M & 0.057 & 0.005 & 8.8 \% \\ 0.491 & 0.293 & 0.024 & 7.8 \% \\ 0.695 & 0.637 & 0.028 & 4.7 \%\end{array}$


$1.0 \mathrm{~N}$

$\begin{array}{llcc}\text { CATECHOL } & \text { SLOPE } & \text { STANDARD } & \text { RELATIVE STANDARD } \\ \text { CONCENTRATION } & & \text { DEVIATION } & \text { DEVIATION } \\ 0.249 M & 0.360 & 0.132 & 36.7 \% \\ 0.492 & 0.752 & 0.051 & 6.8 \% \\ 0.734 & 2.43 & 0.260 & 10.7 \%\end{array}$

The slope of the kinetic decay plots of the EAC is equal to the sum of the forward and backward rate constants for the decay of the EAC as seen in equation (34).
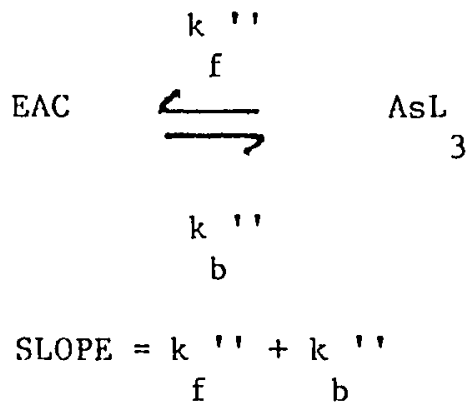

The values of the forward and reverse rate constants can be determined if an additional equation is available containing $k$ '' and $k$ ''. The expression for the equilibrium constant for the pseudo first b order decay of the EAC contains the necessary terms and is seen in equation (35).

$$
\mathrm{K}^{\prime \prime}=\underset{\mathrm{k}}{\mathrm{k}} \underset{\mathrm{b}}{\mathrm{k}} \underset{[\mathrm{EAC}]}{[\mathrm{AsL}]}
$$

Unfortunately the value of $\mathrm{K}^{\prime \prime}$ is not known and so the pseudo first order decay constant, $k$ '', cannot be calculated. The value of the slope for the pseudo first order decay of the EAC is an adequate 
representative of the decay rate. The value of the slope of the kinetic plots increases as the hydrogen ion concentration increases. The dependence of the decay rate on hydrogen ion concentration suggests that hydrogen ion is probably a catalyst for the decay of the EAC. In equation 36 the decay reaction of the EAC to form the predominant equilibrium species, $1: 3$, is presented.

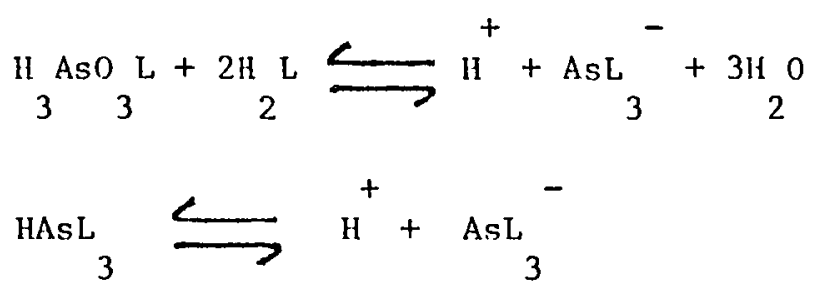

There is not sufficient information to determine the specific role of hydrogen ion in the decay of the EAC and formation of the predominant $1: 3$ species.

DIFFERENTIAL PULSE POLAROGRAPHIC STUDIES OF As(IIL) AND As(V) IN THE PRESENCE OF CATECHOL

The technique of differential pulse polarography, (DPP), was applied to the problem of speciation of inorganic arsenic. This technique has been successfully used for As(III) analysis in water, sewage and sludge samples $(28,34)$. In order to apply this technique to inorganic arsenic speciation the effect of catechol on the polarography of $\Lambda s(I I I)$ and $\Lambda s(V)$ was examined.

The effect of catechol on the polarography of As(III) was found to be small. The first DPP reduction peak for As(III) was shifted by -10 $\mathrm{mV}$ in the presence of $0.5 \mathrm{M}$ catechol. The reduction peak height 
changes by $31 \%$ following the addition of solid catechol to produce a 0.5 M solution. This is a small effect on the polarography of As(III) and the result is an increase in the value of the detection limit of As (III) in catechol solutions.

Typical DPP recordings of the reduction of As(III) are seen in Figure 8. The peak current for the first reduction step is measured and used to prepare a calibration curve. A plot of peak current vs. As(III) concentration is seen in Figures 23 and 24. The detection limit was calculated by the method of Skogerboe et al. (53). The calculated detection limit at the $95 \%$ confidence level is $3.2 \mathrm{ppb}$. This detection limit is larger than the one reported by Myer and Osteryoung for solutions of As(III) without catechol. However, these detection limits are acceptable for environmental applications. The linear range extends from $2.5 \mathrm{ppb}$ to $30 \mathrm{ppm}$, essentially the same as the linear range reported by Myer and Osteryoung (28).

The speciation of inorganic arsenic also requires determination of As $(V)$ concentrations. Typically $A s(V)$ is electroinactive, an exception to this behavior is observed in catechol solutions. As(V) solutions were monitored by polarographic methods in the presence of catechol. Each As(V) analysis requires monitoring the decay of the EAC and using the data collected to calculate the value of 1 which is proportional to the As(V) concentration. Calibration curves were prepared for As(V) solutions. Figure 25 is an example of such a calibration curve. The detection limit calculated by the method of Skogerboe et al. (52) is equal to $0.24 \mathrm{ppm}$ at the $95 \%$ confidence level.

It is obvious that the detection limit for As(V) in catechol is 
much higher than that reported for As(III) in catechol $(0.24$ ppm vs. 3.2 $\mathrm{ppb})$. The poor detection limit is a consequence of the nature of the arsenic(V) catechol interaction. Only a fraction of the As(V) is present as the electroactive species, $(1: 1)$. The remainder of the As(V) is found in higher order complexes which are electroinactive. These polarographic techniques are not sensitive enough for analysis of As(V) in typical environmental samples where As(V) levels are often in the ppb range. 
STRIPPING VOLTAMMETRY

The technique of cathodic stripping voltammetry with copper codeposition was successful for application to speciation of inorganic arsenic. Experiments were conducted to achieve a better understanding of the reactions involved in cathodic stripping with copper codeposition. Then this technique was applied to the problem of inorganic arsenic speciation.

\section{Cathodic Stripping with $\mathrm{Cu}$ Codeposition}

The technique of cathodic stripping with copper codeposition has been applied to As(III) analysis by two research groups $(43,44)$. Both research groups suggest that arsenic and copper form an intermetallic deposit during the plating step of the stripping experiment. Sadana (44) attributed the great sensitivity of his technique to the generation of hydrogen during the stripping reaction. The following two equations were proposed by Sadana (44) for the deposition and stripping of arsenic with copper codeposition.

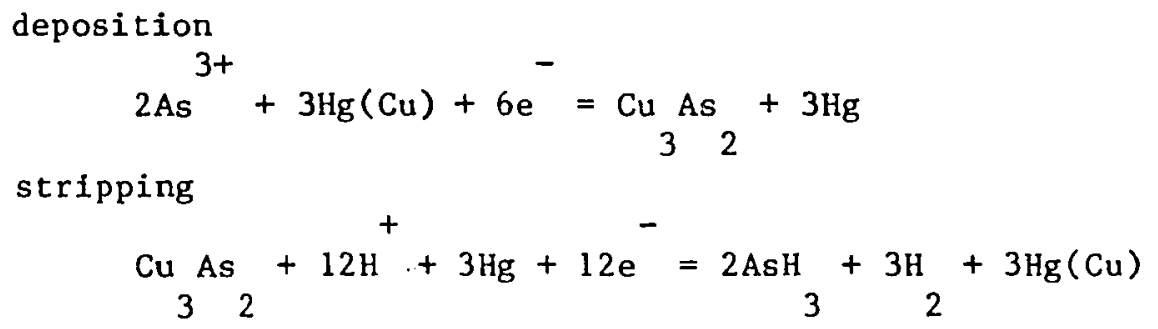

Sadana (44) presents no evidence which demonstrated that hydrogen gas or any other compound was produced during the cathodic stripping of 
arsenic.

The experiments conducted in this study which integrated the cathodic stripping currents for As(III) provide evidence which demonstrates that the stripping peaks produced are indeed enhanced. In addition to the reduction of elemental arsenic to arsine another electron transfer reaction is occuring during the cathodic stripping of the arsenic/copper intermetallic deposit. This additional reduction reaction results in the enhanced current observed in the stripping of the arsentc/copper deposit. The reduction of hydrogen ion to form hydrogen gas is most probably the addizional reaction which occurs during cathodic stripping and results in current "enhancement" and the great sensitivity of these techniques.

The catalysis of hydrogen evolution at mercury electrodes by arsenic has been reported before. In their review of the electrochemistry of arsenic Tomflov and Chomutov (56) report that solutions of arsenic compounds and weak organic acids or salts of cobalt, iron or nickel in neutral electrolytes produce a current peak, often called a polarographic maximum. This maximum has been attributed to catalyzed hydrogen evolution. The polarographic reduction of As(III) in acid solution also produces a maximum which has been attributed to catalyzed hydrogen evolution. In their review of the polarography of arsenic Arnold and Johnson also discuss the presence of maximums which are the results of hydrogen catalysis (57).

This evidence supports the proposal that hydrogen catalysis is the source of the enhanced stripping current for arsenic. The articles cited in the reviews of Tomolov and Chomutov (56) and Arnold and Johnson 
(57) suggest that added metal salts, or metal ion impurities are a cause of the hydrogen catalysis observed in conjunction with arsenic reduction. The presence of an additional metal ion is also needed for the sensitive cathodic stripping methods developed for arsenic. Holak (42) uses Se(IV) and Henze et al (43) and Sadana (44) recommend the use of $\mathrm{Cu}($ II) for cathodic stripping of As(III). Experiments conducted in this study also demonstrated the cathodic stripping of arsenic with codeposition of $\mathrm{Ni}(\mathrm{II}), \mathrm{Pd}(\mathrm{II})$, and $\mathrm{Fe}(\mathrm{III})$. The added metal ions are reduced at the mercury electrode during the deposition step along with As(III) reduction. The arsenic and added metal combine to form an intermetallic of uncertain stoichiometry $(43,44)$. The intermetallic overcomes the two limitations of elemental arsenic deposits on mercury. The first limitation of elemental arsenic deposits on mercury is their complete insolubility (58). The arsenic intermetallic, on the other hand, appears to be soluble in mercury. Little is known about such termetallic systems of arsenic, copper and mercury. The soluble arsenic/copper intermetallic allows the concentration of arsenic into the mercury electrode. The concept of intermetallic formation with copper in stripping analysis has been explored before for elements such as zinc, lead and cadmium $(59,60,61)$. Fresh copper deposits in mercury resemble floating islands of copper which slowly dissolve into the mercury. Arsenic probably deposits onto these copper islands and forms a soluble intermetallic in a similair manner to that reported for other elements deposited with copper $(59,60,61)$.

Arsenic is a metalloid and semiconductor, these characteristics result in arsenic's second limitation. If an elemental, nonconducting 
film of arsenic forms on the working electrode further deposition and accumulation of arsenic would not be possible. This nonconducting film would prevent the concentration of arsenic into the working electrode and remove the effectiveness of cathodic stripping analysis. The codeposition of another metal, such as copper, results in an intermetallic deposit which is rich in a conducting metal. The intermetallic is conducting, soluble in mercury and its formation results in successful cathodic stripping.

It is the stripping of the arsenic intermetallic deposit which results in hydrogen catalysis. During the stripping of the intermetallic deposit the arsenic is further reduced to arsine and the other metal is left behind in the working electrode, as illustrated in equation 39 for copper. The copper or other metal which remains on the electrode after the intermetallic is stripped of arsenic is present as a small metallic island fioating on the mercury surface $(59,60)$. The metal island is exposed to the solution at a fairly negative electrode potential, $(-0.72 \mathrm{~V}$ to $-0.9 \mathrm{~V}$ versus $\mathrm{Ag} / \mathrm{AgCl}$ reference electrode). In the case of copper, this electrode potential is sufficiently negative for hydrogen evolution. Unlike the surrounding mercury electrode, whose hydrogen overpotential is quite large, the copper islands, which have a smaller hydrogen overpotential, reduce hydrogen ions to produce hydrogen gas. The evolution of hydrogen ceases as the copper islands dissolve into the surrounding mercury electrode. In this context the "catalysis" of hydrogen evolution is a misnomer. The presence of the more noble metal, copper, on the mercury surface permits facile hydrogen evolution when compared to the inhibition of hydrogen reduction on 
mercury.

The experiments performed with the metal-amalgam electrodes support this scenario. Cathodic stripping of arsentc was successfully performed at copper-amalgam electrodes. The size of the stripping peak increased if the deposition step was preceeded by oxidative conditioning at $+0.0 \mathrm{~V}$ versus the $\mathrm{Ag} / \mathrm{AgCl}$ reference electrode prior to application of the deposition potential. The oxidative conditioning of the copperamalgam electrode introduced $\mathrm{Cu}$ (II) fons into the analysis solution. The solution phase $\mathrm{Cu}(\mathrm{II})$ deposits along with the arsenic during application of the deposition potential. The intermetallic formed during deposition contains the copper-amalgam of the working electrode plus the fresh copper deposited with the arsenic. This intermetallic deposit is richer in copper than the intermetallic deposit formed without oxidative conditioning. The copper amalgam formed from the copper electrode and the mercury film has a copper concentration of approximately $0.002-0.003 \%$ copper (58) which is the reported solubility of copper in mercury. The richer copper deposit formed following oxidative conditioning may be super saturated with copper and is a more effective catalyst for evolution of hydrogen. The cathodic stripping peak for arsenic increases in height and area when the copper-amalgam electrode is subjected to oxidative conditioning. In essence, the more copper in the intermetallic deposit the more effective the hydrogen catalysis.

This interpretation was confirmed when cathodic stripping was performed on As(III) solutions with and without catechol at a copperamalgam electrode. The cathodic stripping peak current decreased by 
approximatley $45 \%$ after solid catechol was added to the analysis solution. The added catechol apparentiy complexes the oxidized copper found in the solution phase, which is normally available for codeposition with the arsenic. The resultant intermetallic which forms from catechol solutions has less copper than intermetallics formed in solutions free of catechol and the resultant hydrogen catalysis observed during cathodic stripping is reduced.

Both Henze et al. (43) and Sadana (44) report that the cathodic stripping peak height changes as a function of $\mathrm{Cu}(\mathrm{II})$ concentration. Sadana (44) examined the low concentration range of $0 \mathrm{ppm}$ to $7 \mathrm{ppm}$ Cu(II). Essentially no stripping current was observed until the concentration reached $3 \mathrm{ppm} \mathrm{Cu}(\mathrm{II})$. Above $7 \mathrm{ppm}$ the stripping current rapidly increased with copper concentration. Henze et al. (43) reported that the stripping peak current increased with copper concentration until approximately $110 \mathrm{ppm} \mathrm{Cu}(\mathrm{II})$. At this $\mathrm{Cu}$ (II) concentration the stripping peak current no longer changed with increasing copper concentration. Similair results were obtained in this study. This evidence supports the contention that the cathodic stripping of arsenic produces a stripping product whose quantity is related to the amount of copper present during deposition and stripping. The cathodic stripping peak results from reduction of elemental arsenic to arsine and evolution of hydrogen gas. The more solution copper present in the analysis mixture the richer the deposit is in copper, and the greater the hydrogen evolution.

Polarographic studies of arsenic have reported polarographic maxima and hydrogen catalysis $(28,31,32)$. In Figure 8 a differential 
pulse polarogran of $3 \mathrm{ppm} \mathrm{As}$ (III) in $1.0 \mathrm{M} \mathrm{HClO}$ and $0.5 \mathrm{M}$ catechol shows a small maximum at $-0.76 \mathrm{~V}$ versus the $\mathrm{Ag} / \mathrm{AgCl}$ reference electrode. This maximun occurs at a potential between the reduction of As(III) to $\mathrm{As}(0)$ and the reduction of $\mathrm{As}(0)$ to $\mathrm{AsH}$. In this intermediate potential range elemental arsenic accumulates at the working electrode along with any trace impurities with more positive reduction potentials than $-0.55 \mathrm{~V}$ versus the $\mathrm{Ag} / \mathrm{AgCl}$ reference electrode.

The accumulation of elementai arsenic and trace impurities at the electrode in the potential range of $-0.55 \mathrm{~V}$ to $-0.85 \mathrm{~V}$ versus the $\mathrm{Ag} / \mathrm{AgCl}$ reference electrode results in intermetallic deposits similair to the arsenic/copper deposits formed in the cathodic stripping techniques discussed above. During the polarographic analysis cathodic scans are applied to the electrode at typical rates of $5-10 \mathrm{mV} / \mathrm{s}$. During the lifetime of each drop, which is typically 1 to 5 seconds long, a deposit of arsenic and trace impurities forms when the electrode potential is in the following range, -0.55 to $-0.85 \mathrm{~V}$ versus the $\mathrm{Ag} / \mathrm{AgCl}$ reference electrode. The polarographic maximum, observed in the potential range of $-0.70 \mathrm{~V}$ to $-0.95 \mathrm{~V}$ versus the $\mathrm{Ag} / \mathrm{AgCl}$ reference electrode, occurs when the potential is sufficiently negative to form an arsenic intermetallic deposit and further reduction of the deposit occurs as the cathodic scan continues during the lifetime of the drop. This process produces the polarographic maximum which results from catalysis of hydrogen evolution in a simflair manner to the process observed in cathodic stripping with copper codeposition.

When the applied potential 1 s more negative than $-0.95 \mathrm{~V}$ versus the $\mathrm{Ag} / \mathrm{AgCl}$ reference electrode the solution $\mathrm{As}($ III) is directly reduced 
to arstne without forming an intermediate elemental deposit. Without the formation of an intermetallic deposit catalysis of hydrogen evolution is not observed. The appearance of the polarographic maximum is dependent on As(III) concentration. The polarographic maximum is only observed at concentrations above $10 \mathrm{uM}, 0.75 \mathrm{ppm}$ As(III). At lower arsenic levels the arsenic deposit formed is too dilute to form the intermetallic necessary for hydrogen evolution. This is to be expected in polarographic methods where each mercury drop electrode only exists for a few seconds at most. During the short lifetime of each drop only an insignificant amount of the total solution arsenic is reduced, forming a very small surface concentration of arsenic. Apparently the arsenic deposit must reach a certain concentration for intermetallic formation and the resultant maximum and catalysis of hydrogen evolution to be observed.

Application of Cathodic Stripping Voltammetry to As(V) Analysis

The technique of cathodic stripping with copper codeposition was applied to the problem of inorganic arsenic speciation. In order to determine the concentration of both As(III) and As(V) the techniques of Henze et al. (43) and Sadana (44) were modified. The necessary modifications and use of the resultant technique for speciation of inorganic arsenic are discussed below.

The determination of $\mathrm{As}(\mathrm{V})$ by electrochemical means requires use of catechol as a complexing agent. Catechol has a detrimental effect on the cathodic stripping techniques of Henze et al. (43) and Sadana (44). The effects of catechol on these cathodic stripping techniques was 
eliminated by use of $\mathrm{Cu}(I)$ as the source of copper for codeposition and hydrochloric acid as the hydrogen ton source and supporting electrolyte. Chloride ion from the hydrochloric acid and $\mathrm{Cu}(\mathrm{I})$ combine to form a stable complex, $\mathrm{CuCl}$, which resists reaction with catechol. For 2

successful application of cathodic stripping with copper codeposition to As(V) determination the analysis conditions require $\mathrm{Cu}(\mathrm{I})$ and hydrochloric acid.

$\mathrm{Cu}(\mathrm{I})$ has been used as a reducing agent for conversion of As(V) into As(III) (53) under strongly acid conditions. The reduction of As(V) to As(III) by $\mathrm{Cu}(\mathrm{I})$ does not occur under the conditions of $1.0 \mathrm{~N}$ $\mathrm{HCl}$ recommended here.

Method for Speciation of Inorganic Arsenic

The method developed in this study for the speciation of inorganic arsenic is performed in two steps. The concentration of As(III) is determined from the results of cathodic stripping analysis performed in the first step. Catechol is then added to the analysis mixture in the second step and cathodic stripping is again conducted. The results from both steps of the experimental procedure provide data from which the concentration of $A s($ III) and As(V) can be determined.

The concentration of As(III) is determined in step I by cathodic stripping with copper codeposition. The analysis conditions chosen are very similair to those recommended by Sadana (44) and Henze et al.(43). Samples were acidified to $1.0 \mathrm{~N} \mathrm{HCl}$. Then $\mathrm{Cu}(\mathrm{I})$ was added to the sample to produce a concentration of $8 \mathrm{ppm}$. Cathodic stripping was performed on this solution by application of a deposition potential of $-0.4 \mathrm{~V}$ 
versus $\mathrm{Ag} / \mathrm{AgCl}$ reference electrode for 1 minute followed by a cathodic scan performed at the rate of $10 \mathrm{mV} / \mathrm{s}$. The resultant cathodic stripping peak is proportional to the As(III) concentration.

The concentration of $A s(V)$ is calculated from the results of step II. The analysis mixture from step I is treated with a catechol solution resulting in a sample that has the following characteristics; $1.0 \mathrm{~N} \mathrm{HCl}, 8 \mathrm{ppm} \mathrm{Cu}(\mathrm{I})$ and $0.5 \mathrm{M}$ catechol. Cathodic stripping analysis is performed on this sample in the same manner as step I exactly 30 seconds after mixing the appropriate solutions together. The stripping peak current is proportional to the sum of the As(III) and As(V) concentrations. The As(III) contribution is subtracted from the step II signal and the resultant quantity is proportional to the As(V) concentration. The development of As(III) calibration curves and As(V) calibration curves is discussed below.

Arsenic (III) contributes to the cathodic stripping peaks recorded in each step of this speciation procedure. The cathodic stripping peak current measured for $\mathrm{As}$ (III) in step I is directly proportional to the As(III) concentration in the range of $0 \mathrm{ppb}$ to about $150 \mathrm{ppb}$, as seen in Figures 28 and 32. Samples containing only As(III) were examined with the complete speciation procedure. Of particular interest is the change in stripping peak current for solutions of As(III) when step I and step II are performed. The stripping peak current measured for As(III) in step II is again proportional to concentration in the range of $0 \mathrm{ppb}$ to approximately $100 \mathrm{ppb}$. The value of the stripping peak current is expected to be $48 \%$ smaller as the result of dilution which occurs in step II. The actual change in the stripping peak current is 
not exactly $48 \%$ but instead varies as the As(III) concentration changes. The change in stripping current from step I to step II cannot be predicted by the $48 \%$ reduction in arsenic concentration. The source of this unpredictability is unknown. Two calibration curves, such as Figures 28 and 29 are necessary for determining the As(III) stripping peak contributions in step II measurements. The As(III) concentration determined from step I results and a calibration curve, Figure 28, is then multiplied by 0.52 in order to calculate the As(III) concentration in the step II analysis solution. The step II calibration curve for As(III) alone is then used to determine the As(III) contribution to the total step II stripping current. When the As(III) contribution is subtracted from the total step II result the remaining current is attributed to any As(V) in solution.

The analysis of As(V) solutions by the two step cathodic stripping technique produced a calibration curve for step II, as seen in Figure 31. The analytical range for $A s(V)$ is from approximately $20 \mathrm{ppb}$ to 150 $\mathrm{ppb}$ in step II solutions. This analytical range corresponds to an As(V) concentration range of $40 \mathrm{ppb}$ to $300 \mathrm{ppb}$ in the original sample. This analytical range for As(V) determinations is a great improvement in sensitivity when compared to analysis by differential pulse polarography performed in catechol solution.

Figures $32,33,34$, and 35 illustrate the use of this two step technique for analysis of a variety of inorganic arsenic mixtures. The results illustrate the analysis of solutions with identical As(V) concentrations and different As(III) concentrations in Figures 32 and 33. The As(V) does not contribute to the stripping peak current 
measured in step $I$, as can be seen in Figure 32. In step II, the stripping current measured for each solution contains the As(V) contribution, as expected.

Figures 34 and 35 illustrate the use of this technique for analysis of solutions of different $A s(V)$ levels and identical As(III) concentrations. In Figure 34 it can be seen that the step I stripping peak of each inorganic arsenic mixture was essentially the same even though each sample contains different As(V) concentrations. The step II results illustrated in Figure 35 demonstrate the application of this technique to inorganic arsenic mixture analysis.

The detection limit calculated for As(III) from analysis of a mixture of inorganic arsenic by the two step cathodic stripping method was found to be $2.2 \mathrm{ppb}$ at the $95 \%$ confidence level as calculated by the method of Skogerboe and Grant (54). The detection limit calculated for As (V) in solutions of inorganic arsenic is $28.4 \mathrm{ppb}$ As(V) at the $95 \%$ confidence level (54). This detection limit calculated for As(V) determination in step II corresponds to an original sample concentration of $49 \mathrm{ppb}$. The detection limit for As(V) determined by analysis with the two step technique is an improvement over other reported electrochemical methods which measure As(V) indirectly $(35,37)$. Unfortunately this technique is not sensitive enough for analysis of solutions with $\mathrm{As}(\mathrm{V})$ concentrations in the low ppb range. This sensitivity is necessary for study of many environmental samples. The detection limit is low enough to evaluate the As(V) concentration in samples which exceed the EPA drinking water standard (2). Studies should be conducted to characterize the inorganic arsenic speciation of 
such samples which exceed the primary drinking water: standard.

Additional experiments can be conducted to atempt to lower there detection limit of this two step stripping technique. An obvious area of study is the addition of a concentration step prior to cathodic stripping analysis. The effect of an added concentration step on the inorganic arsenic spectation can be easily monitored by this technique. Unfortunately the addition of a concentration step, as observed for many other arsenic speciation methods $(8,18,19,20)$, increases the length of analysis time and defeats one of the goals of this study, to develop a rapid method for inorganic arsenic speciation.

\section{Conclusions}

In addition to the development of a speciation method for inorganic arsenic in this study, information about the As(V)-catechol complexes was obtained. The electroactive complex was identified as the $1: 1$ species and the value of the formation constant of the $1: 1$ complex was determined. This study is one of the first applications of electrochemical methods for determination of the formation constants of a transient species such as the $1: 1$ As(V)-catechol complex. The use of electrochemical methods for quantification of any material via a transient species such as the 1:l As(V)-catechol complex is a unique approach. It may also be possible to examine formation of the higher order As(V)-catechol complexes with electrochemical methods through the decay of the EAC.

Study of the cathodic stripping of arsenic with metal codeposition revealed a number of clues about the mechanism of this phenomenom. 
Codeposition of arsenic and a second metal onto mercury electrodes forms a soluble conducting intermetallic. In cathodic stripping the arsenic in the intermetallic is further reduced forming arsine and leaving behind tiny metal islands at the mercury surface. These tiny metal islands are formed at sufficiently negative potentials to permit evolution of hydrogen gas on their surfaces until the metal islands dissolve into the surrounding mercury electrode. Similar behavoir is observed in polarographic analysis of As(III) solutions and As(V)catechol solutions with concentrations above $0.75 \mathrm{ppm}$. The polarographic maximum reported $(28,32,45,56,57)$ can be attributed to hydrogen evolution which occurs from similair intermetallic deposits which form between arsenic and trace metal impurities in the electrolyte. Further study is needed in this area to examine the stoichiometry of the arsenic intermetallic deposits and the relationship between the stoichiometry and hydrogen gas evolution. Finally conclusive identification of hydrogen gas as the enhancement product of arsenic cathodic stripping with metal codeposition must be performed. This identification could be made with use of rotating ring disk electrodes to examine the stripping products. 


\section{REFERENCES}

1 "Arsenical Pesticides", ACS Symposium Series No. 7, E.A. Woo1son, (Ed.), (1975).

2 Title 40-Environmental Protection, Chapter I-Environmental Protection Agency, National Interim Primary Drinking Water Regulations, subpart B, 144.11 "Maximum Contaminant Levels for Inorganic Chemicals" Code of Federal Regulations: National Archives of the United States: [Supt. of Docs., U.S. G.P.0., distributor,] U.S. Govt. Print. Off., Washington D.C. 20402.

3 Title 29-Labor, Chapter XVII-Occupational Safety and Health Administration. Part 1910.1018, Subpart $Z$, "Toxic and Hazardous Substances: Inorganic Arsenic", Code of Federal Regulations: National Archives of the United States:[Supt. of Docs.,U.S. G.P.O., distributor] U.S. Govt. Print. Off., Washington D.C. 20402.

4 IARC Monographs, 23, "Arsenic and Arsenic Compounds", 114(1980).

5 Chemical and Engineering News, 60(15), 4(1982).

6 ibid. $60(3), 1$ (1982).

7 ibid. oct. 5,1981 , page 4 .

8 Braman, R.S. and C.C.Foreback, Science, 182,1247(1973).

9 Wood, J.M., Science, $183,1049(1974)$.

10 And reae,M.0., Deep-Sea Research, 25,391(1978).

11 Cherry,J.A., A.U. Shaik, D.E.Tallman and R.V.Nicholson, Journal of Hyd rology, 43, 373 (1979).

12 "Standard Methods for the Examination of Water and Waste Water," American Public Health Association, Water Pollution Control Federation, 1976, method 404-A, 404-B.

13 Amnual Book of ASTM Standards, part 31 Water, American Society for Testing and Materials, 1916 Race Street, Philadelphia, Pennsylvania, 19103, Method 12972-28.

14 Stauffer,R.E., Anal.Chem., 55, 1205(1983). 
15 Freeman, H., J.F.Uthe, and B.Flemming, Atomic Absorption Newsletter, $\underline{15}, 49(1976)$.

16 Holak, T., Anal.Chem•, 41, $1712(1969)$.

17 A.Yasui, C.Tsutsami, and S.Toda, Agric. Biol. Chem.,42, 2139(1978).

18 Brinkman,F.E., K.L. Jewett, W.P. Iverson, K.J. Irgolic, K.C. Ehrhardt, and R.A. Stockman, Journal of Chromatography, 191,31(1980).

19 Iverson, D.G., M.A. Anderson, T.R. Holm, and R.R. Stanforth, Environmental Science and Technology,13,1491)1979).

20 Woolson,E.A., and N. Aharonson, J.Assoc.Off.Anal. Chem., 63, 532(1980)

21 Grabinski, A. A., Anal.Chem., 53,966(1981).

22 Ricci,G.R., L.S.Shepard,G.Coloros and N. E. Hester, Analytical Chemistry, $\underline{53}, 610(1981)$.

23 Uthus, E.0., M.E.Collings, W.E.Cornatzer, F.H.N1elsen, Anal• Chem., $\underline{53}, 2221(1981)$.

24 Goulden,P.D., D.ll.J.Anthony, K.D. Austen, Anal. Chem., 53, 2027 (1981).

25 de Oliveira,E., J.W. Mclaren, and S.S. Berman, Anal. Chem., 55, 2047 (1983).

26 Morita,M., T.Uehiro, and K.Fuwa, Anal. Chem., 53, 1806 (1981).

27 Odanaka,Y., N.Tsuchiya, O.Matano, and S. Goto, Anal. Chem., 55, 929 (1983).

28 Myer, D.J., M.E. Osteryoung, Anal. Chem.,45, 267 (1973).

29 Elton,R. and W.E.Geiger,Jr., Anal. Lett., 9, 665 (1976).

30 Bess, R.C., K.J.Irgolic, J.E.Flannery, and T.H. Ridgway, Anal. Lett., $\underline{9}, 1091(1976)$.

31 Arnold, J.P. and R.M. Johnson, Talanta, 16, 1119(1969).

32 Meites, Louis, J.Am. Chem. Soc., 76, 5927(1954).

33 Arnold, J.P. and R.M. Johnson, Chemistry and Industry, Sept. 23, 1976, 1602 . 
34 Myer,D.J., M.E.Heimbrook, J.0steryoung, and S.M.Morrison, Environmental Letters, 5,53 (1973).

35 llenry,F.T., T.0.Kirch, and T.M.Thorpe, Anal. Chem., 51, 215 (1979).

36 Cox,J.A. and P.J.Kulesza, Anal. Chem., 56, 1021 (1984).

37 Henry,F.T. and T.M.Thorpe, Anal. Chem., 52, 80 (1980).

38 Forsberg,G., J.W.O'Laug1in, R.G.Megargle, and S.R.Koirtyohann, Anal. Chem., 47, 1586 (1975).

39 C.W.Whang, J.A.Page, G.van Loon, and M.P.Griffin, Anal. Chem., 56, $539(1984)$.

40 Davis, P.H., G.R.Dulude, R.M.Griffin, W.R.Mlatson, and E.W. Zink, Anal. Chem., 50, 137 (1978).

41 Lee, S.W. and J.C.Meranger, Anal. Chem., 53, 130 (1981).

42 Holak, W., Anal. Chem., 52, 2189 (1980).

43 Henze,G., A.P.Joshi, R. Neeb, Fresenius Zeitschrift fur Analyt Chemie, 300,267 (1980).

44 Sadana, R.S., Anal. Chem., 55, 304 (1983).

45 White,M.C., and A.J.Bard, Anal. Chem., 36, 61 (1966).

46 Roe, D.K., personal communication.

47 Haak, R., PSU Masters Thesis.

48 Votava,J., and I1. Bartusek, Collection Czechoslav. Chem. Commun., $\underline{42}, 620(1977)$.

49 Larkins,Jr.,T.H., C.E.Martin, and M.M. Jones, Inorganic Chemistry, 2, $554(1963)$.

50 Rais, J., P.Selucky, and S. Drazanova, Inorganic Nuclear Chemistry, 33 , 3087 (1971).

51 Bard, A.J. and L.R. Faulkner, "Electrochemical Methods", John Wiley and Sons, Inc., New York, 1980.

52 Nicholson, R.S. and I. Shain, Analytical Chemistry, 36, 706(1964).

53 Simon,R.K., G.D.Christain, and W.C.Purdy, Amer.J.Clin.Patho1.,49, 207 (1968). 
54 Skogerboe, R.K. and C.L. Grant, Spectroscopy Letters, 3, 215(1970).

55 Dean J. A. Editor, "Lange's Handbook of Chemistry", 13th edition, McGraw Hill Book Company, New York, 1985.

56 Tomilov,A.P. and N.E.Chomutov,"Encyclopedia of Electrochemistry of the Elements", Volume 2, Chapter 2, "Arsenic", A. J. Bard and H. Lund; Editors, Marcel Dekker Inc., New York, 1979.

57 Arnold, J.P. and R.M.Johnson, Talanta, 16, 1191(1969).

58 Hansen, M. and $\mathrm{K}$. Anderk, "Composition of Binary Alloys", second edition, pg. 588, McGraw-Hill, New York, 1958.

59 Wise,J.A., D.A.Roston, and W.R. Heineman, Analytica Chimica Acta $, \underline{154}, 95(1983)$.

60 Neiman, E.YA., L.G.Petrova, V.I. Ignatov, and G.M.Dolgopolova, Analytica Chimica Acta, 113, 277(1980).

61 Shuman, M.S., and G.P.Woodward,Jr., Analytical Chemistry, 48, $1979(1976)$. 